\title{
Modelling medieval masonry construction: taxa-specific and habitat-contingent Bayesian techniques for the interpretation of radiocarbon data from Mortar-Entrapped Relict Limekiln
} Fuels

\author{
Mark Thacker* ${ }^{*}$
}

\begin{abstract}
Using data from simulated and actual case studies, this paper assesses the accuracy and precision of Bayesian estimates for the constructional date of medieval masonry buildings, generated from the radiocarbon evidence returned by different assemblages of wood-charcoal mortar-entrapped relict limekiln fuel (MERLF). The results from two theoretical studies demonstrate how Bayesian model specifications can be varied to generate a chronologically continuous spectrum of distributions from radiocarbon datasets subject Inbuilt Age (IA). Further analysis suggests that the potential for these distributions to contain the date of the constructional event depends largely upon the accuracy of the latest radiocarbon determination within each dataset, while precision is predicated on dataset age range, dataset size and model specification. These theoretical studies inform revised approaches to the radiocarbon evidence emerging from six culturally important Scottish medieval masonry buildings, each of which is associated with a wood-charcoal MERLF assemblage of different botanical character. The Bayesian estimates generated from these radiocarbon datasets are remarkably consistent with the historical and archaeological evidence currently associated with these sites, while age range distributions suggest the IA of each MERLF assemblage has been constrained by the taxa-specific and environmentally contingent lifespans and post-mortem durabilities of the limekiln fuel source. These studies provide further evidence that Bayesian techniques can generate consistently accurate chronological estimates for the construction of medieval masonry buildings from MERLF radiocarbon data, whatever the ecological provenance of the limekiln fuel source. Estimate precision is contingent upon source ecology and craft technique but can be increased by a more informed approach to materials analysis and interpretation.
\end{abstract}

Keywords: Bayesian, Buildings, Charcoal, Ecology, Masonry, Medieval, Outlier, Radiocarbon

\section{Introduction}

Scholars across the world often face significant challenges in ascribing constructional dates to masonry buildings with enough precision to enable meaningful interdisciplinary environmental or historical discourse.

\footnotetext{
*Correspondence: mark.thacker@stir.ac.uk
}

University of Stirling, Stirling, UK
The independent evidence returned by radiocarbon analysis of mortar-entrapped relict limekiln fuel (MERLF) fragments can usefully inform these chronological ascriptions, and the sedimentary context within which these materials survive presents some valuable characteristics to facilitate interpretation of that data. Firstly, the durability of lime mortars can allow them to survive in-situ for hundreds and sometimes thousands of years in upstanding masonry contexts, even if the walls within
Springer Open

The Author(s) 2021. Open Access This article is licensed under a Creative Commons Attribution 4.0 International License, which permits use, sharing, adaptation, distribution and reproduction in any medium or format, as long as you give appropriate credit to the original author(s) and the source, provide a link to the Creative Commons licence, and indicate if changes were made. The images or other third party material in this article are included in the article's Creative Commons licence, unless indicated otherwise in a credit line to the material. If material is not included in the article's Creative Commons licence and your intended use is not permitted by statutory regulation or exceeds the permitted use, you will need to obtain permission directly from the copyright holder. To view a copy of this licence, visit http://creativecommons.org/licenses/by/4.0/. The Creative Commons Public Domain Dedication waiver (http://creativeco mmons.org/publicdomain/zero/1.0/) applies to the data made available in this article, unless otherwise stated in a credit line to the data. 
which they were deposited have been incorporated in later buildings or the structure has become ruinous [13]. Secondly, mortar compositions are historically and environmentally contingent, and material contrasts noted through field survey and lab-based analyses can inform relative phasing interpretations, even where direct stratigraphic relationships are absent (e. g. [4, 5]). And thirdly, the widespread use of wood fuel in pre-industrial limeburning practices has often resulted high concentrations of wood-charcoal MERLF fragment inclusions, and this material has well-recognised radiocarbon dating potential $[6,7]$. These characteristics of durability, distinctiveness, relative ubiquity, and radiocarbon dating potential are all underpinned by a rapid post-depositional mortar set, which not only allows an unequivocal archaeological association between the mortar's constituent components and the surrounding masonry fabric [8], but also precludes infiltration by other materials in later periods. It is crucial for wider interpretation that mortar phasing is correctly understood, and all radiocarbon measurements are accurate [9], but thereafter the determinations returned by all wood-charcoal relict limekiln fuel fragments are expected to calibrate to dates which are no later than the initial deposition of the masonry mortar within which they were entrapped [10-13].

The extent to which these radiocarbon determinations might calibrate to periods which pre-date the construction of the identified masonry phase will be defined by various interrelated factors. The size of the laboratory measurement error margin and the section of the atmospheric calibration curve to which the determination relates are important factors in defining chronological precision, but an allowance must also be made for any 'inbuilt age' (IA) which might separate the average age of the annual tree rings in the MERLF sample (the cells of which rapidly cease exchanging carbon with the surrounding environment after formation) from the constructional event of interest [14-18]. The 'bridging periods' [14] which might contribute to the IA of a sample must be considered on a context-by-context basis and might include post-limekiln factors such as lime transport, mortar maturation and building construction times. But in the absence of historic evidence for the use of pre-prepared charcoal to fuel limekilns [19], the most significant bridging periods are likely to be associated with the loss of outer tree rings from the sample during post-mortem rot, wood conversion, combustion, and/or mortar mixing: periods limited by the pre-kiln growth and storage ages of the selected wood fuels and essentially defined by ecological parameters [7].

The potential IA of every surviving MERLF sample is influenced by its taxa-specific and habitat-contingent lifespan (constraining growth age), habit (influencing trunk and branch wood availability), and post-mortem resilience (constraining storage age). These factors are closely interrelated, since higher resource environments and faster growing taxa are almost universally associated with relatively short lifespan plants characterised by low density wood with poor post-mortem resistance to wood-destroying fungi, and low resource environments and slower growing taxa are widely associated with longer lifespan plants characterised by higher density wood with better post-mortem resistance to wooddestroying fungi [20-25]. In general, therefore, an inverse correlation between the growth-rate of the wood fuel source and the potential IA of the MERLF assemblage generally pertains, and this is determined by taxon and habitat.

These relationships have implications for the archaeological resource at different ecological scales. At the broadest scale, IA potential may be determined by the class of the parent tree and the character of the surrounding biome, since gymnosperms are often characterised by extremely long lifespans [26] and boreal forests can be associated with (post-mortem) coarse woody debris many hundreds of years old [27, 28]. Cold, sheltered and phytogeographically marginal environments are also associated with increased tree longevity in temperate woodlands although, with maximum tree lifespans of 3-400 years pertaining across many old-growth northern hemisphere deciduous forests [29], dendroecological meta-data suggests this is generally more limited than records of particularly (probably very slow-growing) ancient individuals might suggest. Even within this reduced range, however, inter-species contrasts in lifespan and post-mortem resilience can be considerable. In the Atlantic-influenced environments of the UK, dense and comparatively slow-growing angiosperms such as Quercus sp. mature at around 150 years old, can live to over 500 years, and demonstrate high post-mortem durability; while faster-growing shorter-lived genera such as Betula sp. generally mature at around 60 years old, rarely reach 100 years old, and are very rapidly destroyed by fungal attack after death [30-32]. At a finer scale, field reports suggest Betula pendula has a much longer lifespan in the colder climates of central Scotland than elsewhere in these islands [31], and yet the lifespan of 'self-coppicing' Corylus avellana stems (which generally extend to 30-50 years in England) [30], can be limited to 12-15 years on some thin western Scottish soils [33]. Consideration of woodland ecologies in the immediate locality is often useful for evaluation of IA potential, therefore, and particularly in a country such Scotland which is crossed by numerous Northern European phytogeographical boundaries [34, 35]. 


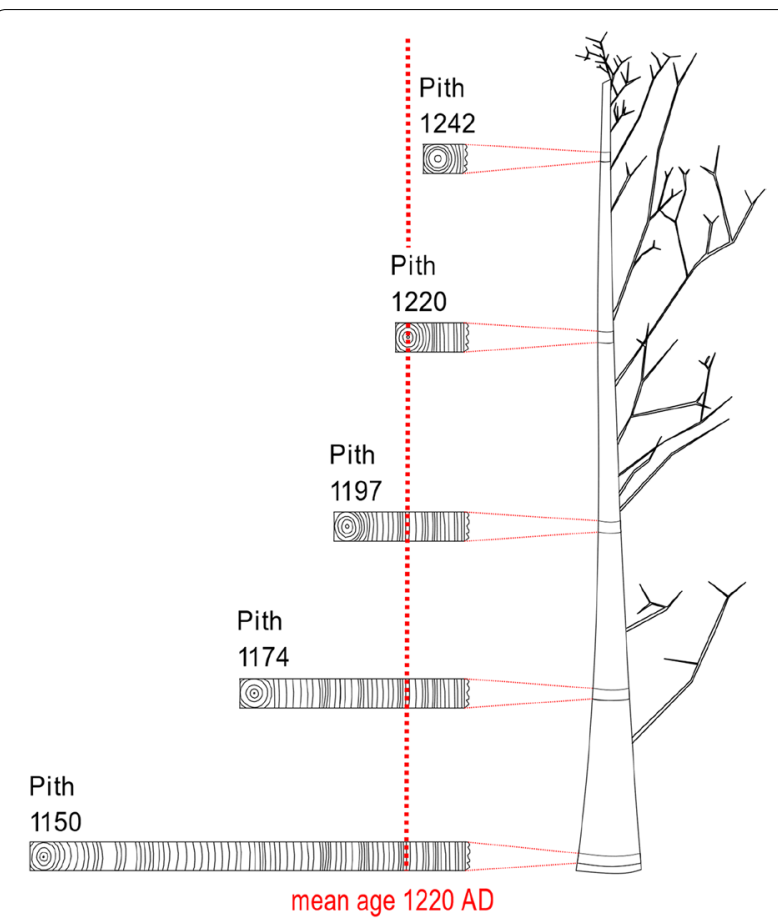

Fig. 1 Stem analysis and radiocarbon dating of annual rings from a theoretical 100-year-old tree displaying exponential growth and felled in c.1250 AD. Background image after Biondi 2020 [25]

Scale of analysis is also important for our interpretations of how the carbon might be distributed within a potential IA range. The distribution of carbon within a woodland exploited for lime-burning fuel will be determined by a complex mix of historically-contingent ecological processes, but although the IA potential of an even-aged stand will tend to increase over time as the population matures and productivity will naturally decline in later years as mortality increases, meta-data gathered from long-term monitoring projects across the globe suggests that aboveground mass growth rates (and so carbon gain) in individual trees generally increases continuously throughout their lifespans [24, 36-38]. Indeed, it has been reported that only $6 \%$ of old-growth forest in the western USA is comprised of trees with trunks of over $100 \mathrm{~cm}$ diameter, and yet this small population contributes a remarkably high ' $33 \%$ of the annual forest mass growth' [36]. This allometric evidence is consistent with reports that more than $50 \%$ of a tree stem's volume typically derives from the outer $30 \%$ of its annual rings [39], and its average radiocarbon age is likely to be approximately a third of its overall lifespan [10] (Fig. 1), while the destruction of old wood during trunk hollow formation [40] and replacement of older branches [41] is likely to sharpen carbon distributions in mature trees still further. Providing the assemblage is representative of the woodland source, therefore, ecological parameters suggest most wood-charcoal samples are likely to calibrate to dates which are equal to or only slightly earlier than their date of deposition [13, 17, 42].

Quantifying the relationship between these radiocarbon determinations and the constructional date of the building from which they were removed is particularly important in historic contexts where wider political, cultural, and environmental processes are understood with reasonably high chronological precision. That the IA of a wood-charcoal fragment is contingent on its botanical character has long been recognised, and half a century ago Tjalling Waterbolk (1971) suggested samples could be usefully divided into three groups whereby: Group A materials deriving from twigs and outermost tree rings would present a radiocarbon 'time difference' which was negligible (< c. 20 years); Group B materials deriving from short-lifespan wood species would present time differences measurable in decades (20-100 years); while Group $\mathrm{C}$ materials deriving from longer lifespan species might result in time differences of over a century [10. See also 11]. ${ }^{1}$ Waterbolk (1971) also acknowledged that the archaeological context from which a sample was recovered might suggest an association with a lower IA Group, and proposed that charcoal fragments from hearths or ovens were likely to be narrowly distributed since 'firewood for daily consumption would have consisted mostly of very young wood' [10], ${ }^{2}$ although the historiography of MERLF materials research reveals contrasting approaches to this issue. Rainer Berger's (1992; 1995) analysis of materials from various pre-Romanesque chapel sites in Ireland, for example, began from a premise that short-lived wood was deliberately selected for limeburning fuel and the calibrated radiocarbon determinations returned by single (probably bulk) MERLF samples were thereby reported as direct standalone constructional estimates [43, 44]. In clear contrast, a re-interpretation of large radiocarbon datasets from masonry buildings in Africa and Asia began from a premise that storage age and re-use in individual wood-charcoal fragments were 'indeterminable' chronological factors, whose interpretation required analysis of multiple samples from each phase $[17,12,45] .^{3,4}$ Notably, this latter assertion followed an article by Patrick Ashmore (1999) which highlighted the dangers of bulking multiple charcoal fragments in a single sample and the challenges of establishing charcoal residence times on archaeological

\footnotetext{
${ }^{1}$ See [10] p. 16.

${ }^{2}$ See [10] p. 22.

${ }^{3}$ See [17] p. 83 .

${ }^{4}$ See [12] p. 15.
} 
sites [18]. But in both instances highlighted above, the radiocarbon distribution of each sample assemblage is not expected to reflect the ecology of a natural woodland source (both are essentially defined by anthropological or technical processes), and there is a concomitant lack of botanical information on the character of the materials under consideration (see also [46-48]).

Ultimately, where the radiocarbon determination returned by a MERLF sample might be associated with significant IA, then circumscription of the chronological period within which the constructional event took place requires a comparative approach. Indeed, multidisciplinary approaches to MERLF radiocarbon data can very usefully bracket the period of building construction where historical or archaeological evidence post-dating that event is also available, since these are effective terminus post quem (TPQ) and terminus ante quem (TAQ) dates, and where the constructional date is already known with some precision then the IA of the MERLF sample or assemblage can be closely quantified [49]. Where multiple radiocarbon determinations are available, however, then Bayesian and other statistical techniques can be used to generate a comparative 'standalone' constructional estimate from the radiocarbon and phasing evidence alone - complete with upper limits which are independent of historical evidence [7]. Once again, such statistical interpretations are generally predicated on a binary distinction between short or long-lived organic materials, although that is most often defined by the distribution of the determinations within the radiocarbon dataset, rather than the botanical character of the samples within the charcoal assemblage. Where samples deposited in a single event have returned determinations so narrowly distributed that a chi-square type test suggests the dataset is statistically consistent at $5 \%$ significance, then the samples can be regarded as effectively contemporaneous, and a more precise Combine average can be generated which is assumed to directly represent their date of deposition [50]. Where determinations from a particular depositional context are not statistically consistent at $5 \%$ significance, however, then a higher level of IA must be suspected, and a different approach is required. In the OxCal calibration programme used throughout this paper, measurements from datasets subject to IA can be grouped into model phases framed by probability distributions known as Start and End Boundaries; and the position of this latter event at the end of the phase (or between phases in a multiphase scenario) may be accepted as a reasonable estimate for the completion of the constructional event [51]. Generating a Last distribution will also provide a probability estimate for the last determination within the series, however, and these measurements can be further constrained to reflect our prior belief that the dataset should be exponentially distributed-in line with allometric data. OxCal offers two main methods by which this might be achieved: a Tau Start Boundary can be selected to impose an exponential 'prior' distribution on the whole phase; or each individual determination can be tagged with an Outlier Probability linked to a separate exponentially distributed Charcoal Outlier Model [13]. The default Charcoal Outlier Model in $\mathrm{OxCal}$ is specified with a 1000 year time-constant to encompass the mean lifespan of an extremely long-lived assemblage, but the logarithmic scale of the model is defined by the actual distribution of the radiocarbon determinations in each dataset and, providing sufficient independent determinations are available, the lowest IA materials are expected to be steeply distributed very close to the exponential asymptote [13, 17].

The assumptions which underpin these interpretive schemes and their general application for MERLF analysis are open to challenge. Ashmore's [18] thesis demonstrated that very short-lived charcoal materials retrieved from various excavated contexts have sometimes returned problematically early radiocarbon determinations, but the storage age potential of MERLF materials is likely to be more limited where wood (rather than charcoal) has routinely been used as a limekiln fuel, and long residence times are largely irrelevant in a mortared masonry context where intrusion is effectively precluded. Recent studies have reported evidence that bark evidence does occasionally survive on wood-charcoal MERLF fragments, and some of the assemblages associated with these fragments have also returned radiocarbon determinations which are statistically consistent at 5\% significance [52], but historical, archaeological, and radiocarbon evidence from across northern Europe and elsewhere suggests that limekilns were often charged with mixtures of different wood taxa which can return statistically inconsistent radiocarbon datasets [7]. In an important piece of work evaluating the accuracy and precision of 'standalone' constructional estimates generated using Bayesian techniques in $\mathrm{OxCal}$, Michael Dee and Christopher Bronk Ramsey (2014) concluded that the Charcoal Outlier Model approach generates the most consistently accurate End Boundary estimates from wood-charcoal datasets, whilst the exponential prior approach generated more precise but occasionally inaccurate distributions [17]. Where the number of determinations from a particular phase is more limited, however, then the Charcoal Outlier approach tends to generate very broad positively skewed End Boundary distributions, which can seem incongruous against the comparatively low mean lifespans of most temperate woodland taxa. The TPQ role 
performed by wood-charcoal MERLF radiocarbon determinations can be of huge value for multidisciplinary interpretation and the effect of model selection on upper limits may be much less important where an early and convincing TAQ is available to truncate these distributions (Fig. 2a-f). Standalone estimate precision, however, is vital for increased interdisciplinary (rather than multidisciplinary) discourse. And while there is also some evidence that reducing the exponential time-constant of the Charcoal Outlier Model to reflect the more limited source material lifespans can constrain End Boundary distributions [49], persistent contrasts in precision with estimates using the exponential prior approach and binary approaches to statistical consistency do not appear to reflect a continuous spectrum of potential IAs predicated on variation in woodland ecologies. Importantly for this paper, however, the accuracy and precision of constructional estimates generated using different Bayesian approaches can also be evaluated using simulated datasets in theoretical models, without initial reference to architectural or historical evidence.

This paper describes a re-evaluation of these Bayesian frameworks, with a concern to further characterise statistical relationships which might pertain between the different limekiln fuel resources exploited during the construction of medieval masonry buildings, and the archaeological potential of any surviving MERLF materials. Following the approach developed by Dee and Bronk Ramsey [17], this work is predicated on Bayesian analysis of simulated and actual radiocarbon datasets subject to varying levels of IA, although a broader range of simulated single-phase datasets, model specifications and generated estimates are considered here. Indeed, two theoretical studies centred on a single date in the medieval period will demonstrate how a chronologically continuous spectrum of distributions can be generated from radiocarbon datasets of varying lifespans and sizes, and different Bayesian model specifications can therefore be employed to maximise constructional estimate precision whilst retaining accuracy. These theoretical results will then inform the modelling approaches applied to the published radiocarbon datasets from six culturally important Scottish medieval buildings (CS1-6), each of which is associated with a MERLF assemblage of contrasting botanical character. Highlighting remarkable levels of consistency between the radiocarbon, historical and ecological data, the paper will conclude that the age range of an in-situ MERLF assemblage does often appear to be constrained by the taxa-specific and environmentally contingent lifespans and post-mortem durabilities of the limekiln fuel source.

\section{Method}

The methodologies presented below detail the processes by which data were generated in two theoretical studies and six case studies. Following previous authors [17], the theoretical studies are essentially circular and pragmatic. Exponentially distributed sets of calibrated radiocarbon dates associated with different IA mean lifespans (hereafter IAT) have been simulated from a particular 'true event' calendar date, and these datasets have then been constrained within various single-phase Bayesian models to assess how the accuracy and precision of newly generated distributions are affected by model specification. In contrast to that previous work, however, a calendar date of $1250 \mathrm{AD}$ has been selected for the true event in each theoretical study, since this correlates with a relatively monotonic section of the radiocarbon calibration curve and occupies a central chronological position relative to the case studies presented later in the paper. The error margin of \pm 35 years on each simulated date was also selected to more closely reflect the data associated with these case studies. An increased range of dataset IAt and model specifications has also been employed, while End Boundary and Last distributions are evaluated for constructional estimate accuracy and precision. All datasets and models have been generated using OxCal 4.4 [51] and are calibrated with the IntCal20 atmospheric calibration curve [53].

\section{Theoretical studies}

In Theoretical Study 1 (TS1), multiple sets of twenty simulated calibrated dates subject to varying levels of IA were generated from a theoretical true event date of 700 $\mathrm{BP} \pm 35$ years $(1250 \mathrm{AD})$. Twenty independent datasets were generated in TS1, with four separate datasets each subject to IAt specified at 10, 50, 100, 200 and 500 years. The number of dates in each dataset which included the true event date has been counted, dataset age ranges have been estimated using the OxCal Difference function to compare the earliest and latest simulated dates [51], and the actual mean lifespan of each dataset has been calculated by finding the sum of the mean values from each individual simulated date. Each dataset has then been situated in a single-phase Bayesian model framed by Start and End Boundaries and subject to a range of different specifications. Run at default Oxcal settings to allow a reasonably fast turnaround of results, these include: (i) a Combine model; (ii) an exponential prior/no outlier model; (iii) an exponential prior/modified Charcoal Outlier Model (with a time-constant modified to the same scale as the IAt specified for the simulated dataset); (iv) an exponential prior/default Charcoal Outlier Model; (v) a uniform prior/no outlier model; (vi) a uniform prior/ 


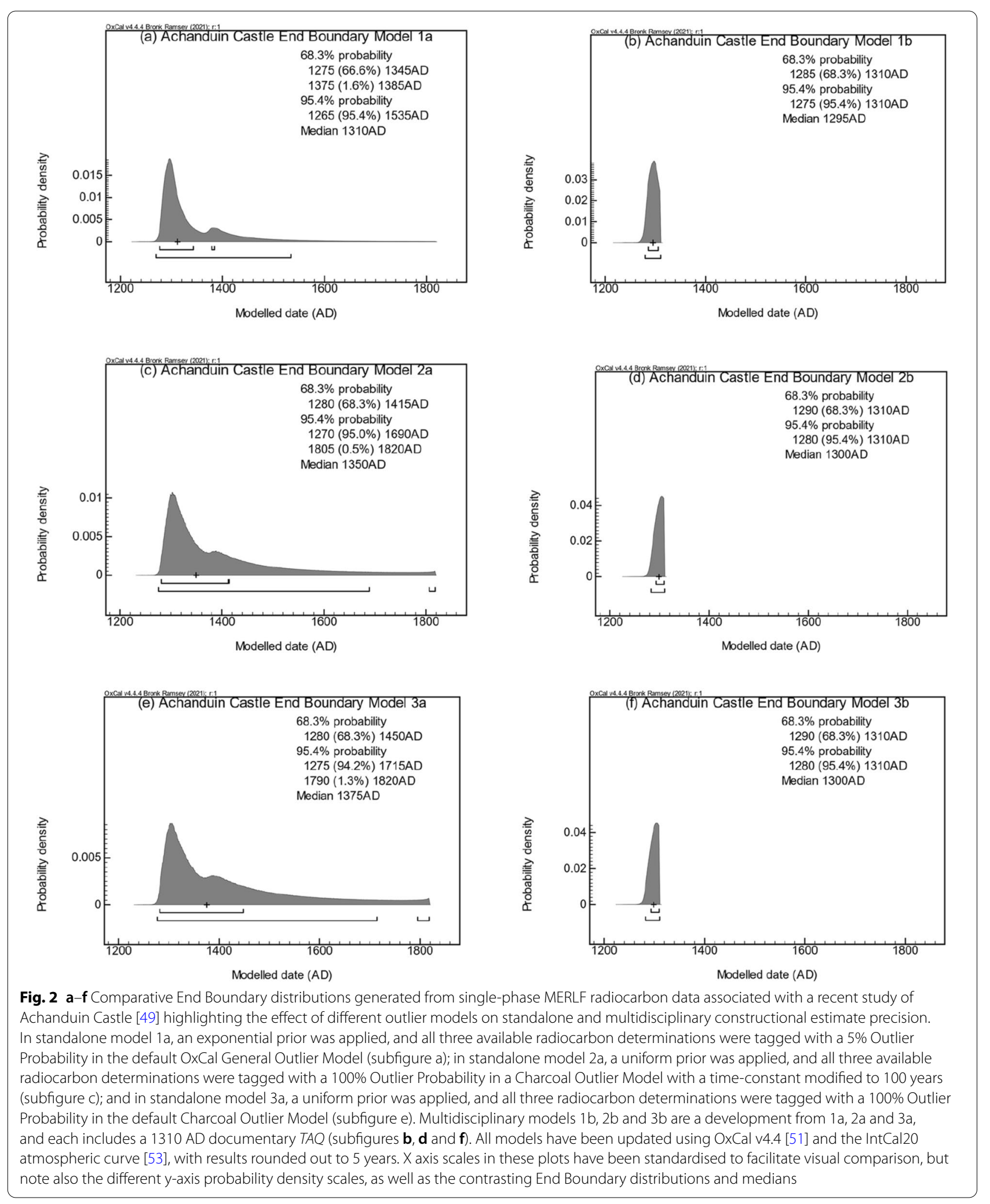


modified Charcoal Outlier Model (with a time-constant modified to the same scale as the specified IAt of the simulated dataset); and (vii) a uniform prior/default Charcoal Outlier Model. In the third and fourth run of each dataset/modelling approach combination, a Last distribution was also generated. All models associated with TS1 are presented in Additional file 1.

In Theoretical Study 2 (TS2), multiple simulated datasets of varying size and subject to varying levels of IA were generated from a theoretical true event date of $700 \mathrm{BP} \pm 35$ years. Forty-five independent datasets were generated in TS2 with three separate datasets associated with an IAt specified to 10, 50, 100, 200 and 500 years and including fifteen, ten and five simulated dates. As in TS1, the number of dates in each TS2 dataset which included the true event date has been counted, the age range has been estimated using the OxCal Difference function to compare the earliest and latest simulated dates, and the actual mean lifespan of each dataset has been calculated by finding the sum of the mean values from each simulated date. These datasets were included in single-phase models framed by Start and End Boundaries and subject to three model specifications including: (i) an exponential prior/no outlier model, (ii) an exponential prior/modified Charcoal Outlier Model (with a time-constant modified to match the IAt specified for the simulated dataset), and (iii) a uniform prior/default Charcoal Outlier Model. All these TS2 models have been run at default settings to allow a reasonably fast turnaround of results, and all include a Last distribution. All models associated with TS2 are presented in Additional file 2.

In Case Sudies 1-6 (CS1-6), the radiocarbon data from six Scottish medieval buildings with wood-charcoal MERLF assemblages comprised of contrasting taxa are re-evaluated. This includes Castle Fincharn main block (CS1), Aros Castle north-west block (CS2), Castle Roy enclosure and tower (CS3), Lochindorb Castle primary enclosure (CS4), Achanduin castle enclosure and hall (CS5), and Lismore Cathedral nave (CS6). The MERLF assemblages associated with the first five of these studies are dominated by charcoal fragments which displayed no surviving terminal ring, bark, or sapwood boundary evidence, and each has been published elsewhere in some detail. Full details of CS6 await publication but is included here since the MERLF assemblage included a Corylus sp. fragment with some terminal ring evidence. The distributions of each of these radiocarbon datasets was investigated using the Ward and Wilson (1978) chisquare type test [50], and an age range was calculated using the Difference function to compare the earliest and latest dates available [51]. The data from each site were then included in a series of single-phase Bayesian models. Run at 1 year resolution and 20,000 Kiterations, these include: a Combine model; an exponential prior/no outlier model; an exponential prior/modified Charcoal Outlier Model; an exponential prior/default Charcoal Outlier Model; and a uniform prior/default Charcoal Outlier Model. The modified Charcoal Outlier Model IA timeconstants specified in CS1-6 have been estimated from published data regarding tree mean lifespan and wood post-mortem resilience data (Table 1). Mean lifespans are rarely reported so working values have been calculated at $33 \%$ of reported maximum lifespans, in line with allometric data, added to which an estimate of post-mortem resilience has been derived from the resistance to wooddestroying fungi according to the (1-5) durability scale applied by British and European Standards [54] and other published reports (Table 1). Where the datasets returned by mixed-taxa assemblages are statistically consistent at $5 \%$ significance (CS5) then these are tagged to a single Charcoal Outlier Model with a time-constant modified to reflect the lowest IAt samples, and where these datasets are not statistically consistent at 5\% significance (CS2 and CS6) then the highest IAt data is used. A Last distribution has been generated in all case study models, and the Last and End Boundary distributions compared with various potential TPQ and TAQ dates (from other types of historical, archaeological or architectural evidence) using the Order function [51]. All models associated with these case studies are presented in Additional file 3. In the interest of brevity, presentation of wider evidence relating to these buildings is kept to a minimum, and readers are encouraged to follow the cited references for more detailed information.

Calibrated date ranges in each theoretical and case study radiocarbon dataset are expressed as cal AD or cal $\mathrm{BC}$ at $95 \%$ and $68 \%$ confidence using upright text and have been rounded out to 10 years [12]..$^{5}$ Modelled age range, End Boundary, and Last distributions are reported as Highest Posterior Density (HPD) interval date ranges at both $68 \%$ and $95 \%$ probability with median values, and these estimates have been rounded out to 5 years and are presented in italics. Generated date ranges in both theoretical studies are regarded as accurate when they include the true event date from which each simulated dataset was generated (i.e. $1250 \mathrm{cal} \mathrm{AD).} \mathrm{The} \mathrm{agreement} \mathrm{indices}$ returned by each model are considered [51, 55], but individual measurements which fall below the accepted $60 \%$ threshold of compatibility have not been removed from theoretical models since to do so would bias results [56].

\footnotetext{
${ }^{5}$ See [12] pp. 42 and 49.
} 
Table 1 Ecological data from UK and Scotland relating to tree taxa from case studies

\begin{tabular}{|c|c|c|c|c|c|c|}
\hline Maximum lifespan & Taxa & Habit & Life cycle & Post-mortem resilience & Working IAT & References \\
\hline 50 Years & Corylus avellana & Shrub & $\begin{array}{l}\text { 'self-coppicing' cycles } \\
\text { of } \leq 30-50 \text { year old stems } \\
\text { in UK. } \leq 12-15 \text { year old } \\
\text { stems on some thin Scot- } \\
\text { tish Atlantic soils }\end{array}$ & $\begin{array}{l}\text { Not included in BSI } \\
\text { standards }\end{array}$ & 20 years & {$[30,33]$} \\
\hline 100 Years & Betula pubescens & $\begin{array}{l}\text { Shrub/Small } \\
\text { Tree }\end{array}$ & $\begin{array}{l}\text { Matures } 50-60 \text { with lifes- } \\
\text { pan } \leq 100 \text { years in UK. } \\
\text { 'Shortest- lived common } \\
\text { tree [in UK] after Aspen.' }\end{array}$ & $\begin{array}{l}\text { 5-Not Durable. Dies rap- } \\
\text { idly and rots quickly. Life } \\
\text { expectancy in ground } \\
\text { contact 'less than } 5 \text { years' }\end{array}$ & 50 years & {$[30-32,54,57-59]$} \\
\hline 200 Years & Betula pendula & Tree & $\begin{array}{l}\leq 100 \text { years in UK generally } \\
\leq 180 \text { years in Central } \\
\text { Scotland }\end{array}$ & $\begin{array}{l}\text { 5-Not Durable. As per } \\
\text { Betula pubescens }\end{array}$ & 100 years & {$[30,31,54,58,60]$} \\
\hline \multirow[t]{2}{*}{600 Years } & Pinus sylvestris & Tree & $\begin{array}{l}\text { Generally aged } \leq 300 \text { years. } \\
\text { Lifespan } \leq 550 \text { years in NW } \\
\text { Scotland }\end{array}$ & $\begin{array}{l}\text { 3-4 moderate-slightly } \\
\text { durable }\end{array}$ & 300 years & {$[54,61,62]$} \\
\hline & $\begin{array}{l}\text { Quercus } \\
\text { robur and petraea }\end{array}$ & Tree & $\begin{array}{l}\text { General ages } \leq 300 \text { years in } \\
\text { UK woodlands. Scot- } \\
\text { tish medieval tim- } \\
\text { ber } \leq 418 \text { years Darnaway } \\
\text { (NE Scotland) }\end{array}$ & $\begin{array}{l}\text { 2-Durable. Sap- } \\
\text { wood } \leq 20 \text { years, heart- } \\
\text { wood over } 50 \text { years }\end{array}$ & 300 years & {$[30,54,63-65]^{\mathrm{a}}$} \\
\hline
\end{tabular}

${ }^{a}$ See [41] p. 130

\section{Results (theoretical studies) Theoretical Study 1 (TS1)}

The twenty simulated datasets generated in TS1 are all exponentially distributed (e.g. Figure 3). There is relatively little variation between the latest simulated date in each dataset, and all include the $1250 \mathrm{AD}$ true event at 95\% confidence (Table 2). Increased dataset IAt is associated with some decrease in latest date age, however, and with a decrease in the number of dates containing the true event at $95 \%$ confidence (from all 20 dates in 10 years IAt dataset M1a run 1, to only a single date in 500 years IAt dataset M1e run 4). An average of 19 simulated dates include the true event in 10 year IAt datasets, 13 in 50 year IAt datasets, 11.5 in 100 year IAt datasets, 5 in 200 year IAt datasets (M1d), and 2.5 in 500 year IAt datasets.

Age range variation in the TS1 datasets is mostly predicated on variation in the earliest simulated dates within each dataset (Table 2). Increased IAt is generally associated with an increase in earliest date age, an increase in date range, and an increase in date range variation. The earliest calibrated dates in all the datasets specified with 10 years IAt are situated in the second millennium $\mathrm{AD}$ and age range variation in this group is limited to between - 35-225 years (M1a run 1) and 20-250 years (M1a run 2). This 10 years IAt group includes the only dataset in TS1 which falls into minus values, and this is the same dataset in which all simulated dates include the 1250 AD true event. In contrast, all earliest dates in the datasets specified with 500 years IAt are situated in the cal $B C$ period, with age range variation between 1315 1640 years (M1e run 3) and 3205-3465 years (M1e run 1). Dataset age ranges in these two lowest and highest IAt groups are distinctive, and the datasets throughout the study are generally consistent with this trend, but there is considerable overlap between individual datasets in adjacent 50, 100 and 200 years IAt groups.

All dataset mean lifespans are earlier than the $1250 \mathrm{AD}$ true event date in TS1 and these values also generally increase in age and variation with increased IAt specification (Table 2). The mean lifespans of the datasets specified with 10 years IAt are narrowly distributed between 1224 and $1242 \mathrm{cal}$ AD, whilst the datasets specified with 500 years IAt present mean lifespans situated in the first millennium AD between 491 and $780 \mathrm{cal} \mathrm{AD}$. The average lifespans within each group are all consistent with this trend and close to expected values $-1229 \mathrm{cal} A D$

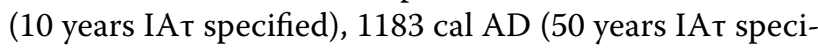
fied), $1156 \mathrm{cal}$ AD (100 years IAt specified), $1039 \mathrm{cal} \mathrm{AD}$ (200 years IAt specified), and $670 \mathrm{cal} \mathrm{AD} \mathrm{(500} \mathrm{years} \mathrm{IA \tau}$ specified)-although there is some overlap in the lifespans of individual datasets in adjacent groups specified to 50,100 and 200 years IAt.

There is a clear relationship between the IAt specified, the distribution of simulated dates, and the date range of each dataset generated in TS1. All four datasets in the 10 years IAt group (M1a runs 1-4) pass the Ward and Wilson (1978) chi-square type test and have generated accurate Combined dates, although three of these models contain four individual dates with low agreement indices 
(a) TS1-M1a-run2

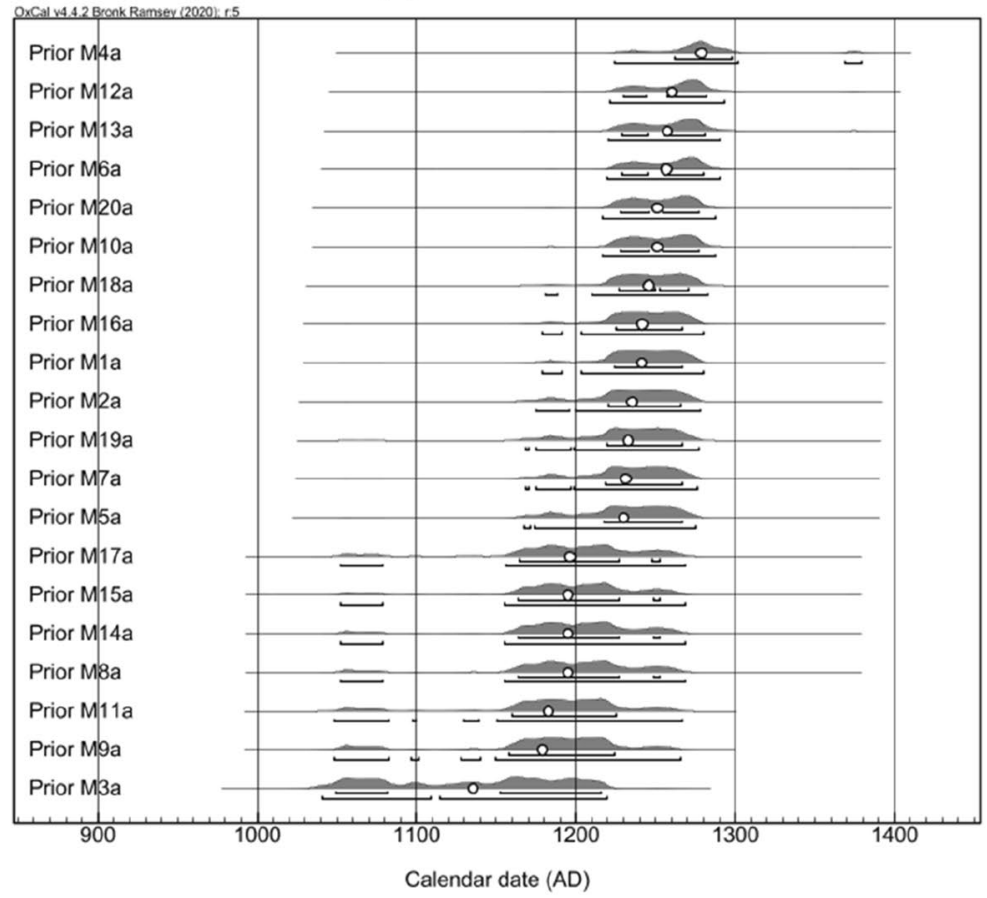

(b) TS1-M1e-run1

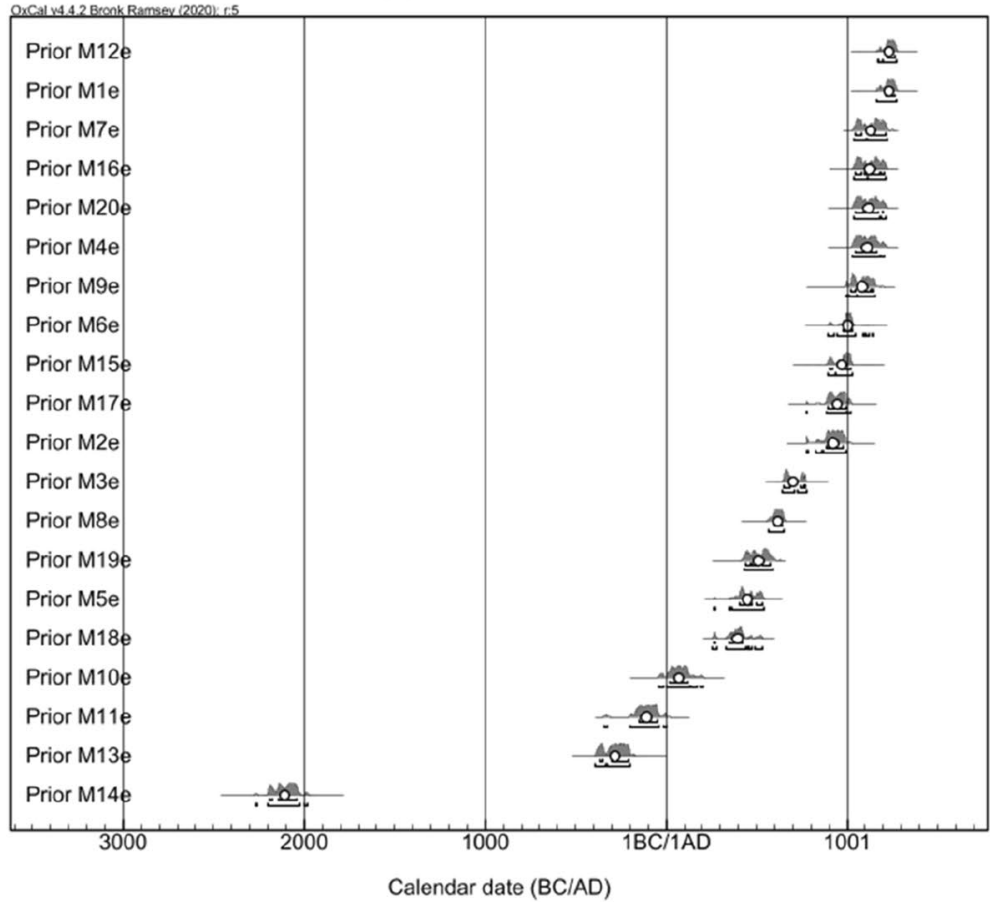

Fig. 3 Selected multiple plots from TS1, illustrating exponentially distributed simulated datasets with specified IAt of 10 years and 500 years Subfigure a presents distribution plots from a dataset specified with 10 years IAt (M1a run 2) with simulated dates ranging from 1040-1220 cal AD (M3a) to 1220-1380 cal AD (M4a) at 95\% confidence, nineteen of which include the true event date of 1250 AD at 95\% confidence. Subfigure $\mathbf{b}$ presents distribution plots from a dataset specified with 500 years IAT (M1e run 1), with simulated dates ranging from 2270 to 1980 cal BC (M14e) to 1160-1280 cal AD (M12e) at 95\% confidence, two of which include the 1250 AD true event date at 95\% confidence. Small circles represent the mean average of each distribution 
Table 2 Selected features of the datasets from TS1. Age range values have been rounded out to 5 years

\begin{tabular}{|c|c|c|c|c|c|c|}
\hline $\begin{array}{l}\text { Specified dataset } \\
\text { IAt and code }\end{array}$ & Run & $\begin{array}{l}\text { Earliest simulated date } \\
\text { (95\% confidence) }\end{array}$ & $\begin{array}{l}\text { Latest simulated date } \\
\text { (95\% confidence) }\end{array}$ & Accurate dates & $\begin{array}{l}\text { Dataset range } \\
\text { (95\% probability) }\end{array}$ & Mean lifespan \\
\hline \multirow{4}{*}{$\begin{array}{l}10 \text { years } \mid \mathrm{AT} \\
\mathrm{M} 1 \mathrm{a}\end{array}$} & 1 & 1050-1280 cal AD (M9a) & 1220-1380 cal AD (M4a) & 20 & $-35-225$ years & $1242 \mathrm{cal} \mathrm{AD}$ \\
\hline & 2 & 1040-1220 cal AD (M3a) & 1220-1380 cal AD (M4a) & 19 & $20-250$ years & $1224 \mathrm{cal} \mathrm{AD}$ \\
\hline & 3 & 1040-1230 cal AD (M12a) & 1220-1300 (M15a) & 18 & $15-235$ years & $1225 \mathrm{cal} \mathrm{AD}$ \\
\hline & 4 & 1040-1230 cal AD (M14a) & 1210-1290 cal AD (M16a) & 19 & $10-230$ years & $1224 \mathrm{cal} \mathrm{AD}$ \\
\hline \multicolumn{4}{|c|}{ Mean Average 10 years IAT } & 19 & $5-235$ years & $1229 \mathrm{cal} \mathrm{AD}$ \\
\hline \multirow{4}{*}{$\begin{array}{l}50 \text { years } \mid \mathrm{A} \tau \\
\mathrm{M} 1 \mathrm{~b}\end{array}$} & 1 & 990-1160 cal AD (M9b) & $1220-1300 \mathrm{cal}$ AD (M14b) & 11 & $85-290$ years & $1180 \mathrm{cal} \mathrm{AD}$ \\
\hline & 2 & 970-1160 cal AD (M14b) & 1220-1390 cal AD (M15b) & 13 & $120-385$ years & $1181 \mathrm{cal} \mathrm{AD}$ \\
\hline & 3 & 1020-1210 cal AD (M11b) & 1220-1300 cal AD (M9b) & 16 & $55-250$ years & $1193 \mathrm{cal} \mathrm{AD}$ \\
\hline & 4 & 890-1120 cal AD (M14b) & 1210-1290 cal AD (M13b) & 13 & $160-380$ years & $1177 \mathrm{cal} \mathrm{AD}$ \\
\hline \multicolumn{4}{|c|}{ Mean Average 50 years IAT } & 13 & $105-325$ years & $1183 \mathrm{cal}$ AD \\
\hline \multirow{4}{*}{$\begin{array}{l}100 \text { years IAt } \\
\text { M1C }\end{array}$} & 1 & 990-1160 cal AD (M4c) & 1220-1380 cal AD (M16c) & 16 & $80-280$ years & $1199 \mathrm{cal} \mathrm{AD}$ \\
\hline & 2 & 680-950 cal AD (M4c) & $1210-1290 \mathrm{cal}$ AD (M9c) & 9 & $310-570$ years & $1145 \mathrm{cal} \mathrm{AD}$ \\
\hline & 3 & 410-570 cal AD (M3c) & 1180-1290 cal AD (M18c) & 10 & $670-845$ years & $1127 \mathrm{cal} \mathrm{AD}$ \\
\hline & 4 & 770-980 cal AD (M17c) & $1210-1290 \mathrm{cal}$ AD (M3c) & 11 & 260-500 years & $1151 \mathrm{cal} \mathrm{AD}$ \\
\hline \multicolumn{4}{|c|}{ Mean average 100 years IAT } & 11.5 & $330-550$ years & $1156 \mathrm{cal} \mathrm{AD}$ \\
\hline \multirow{4}{*}{$\begin{array}{l}200 \text { years IAt } \\
\text { M1d }\end{array}$} & 1 & 400-560 cal AD (M3d) & 1210-1290 cal AD (M18d) & 6 & $680-865$ years & 982 cal AD \\
\hline & 2 & 670-880 cal AD (M2d) & 1160-1280 cal AD (M7d) & 4 & $315-575$ years & 1062 cal AD \\
\hline & 3 & 560-660 cal AD (M6d) & 1180-1290 cal AD (M12d) & 5 & $570-710$ years & $1025 \mathrm{cal}$ AD \\
\hline & 4 & 680-950 cal AD (M5d) & 1170-1290 cal AD (M18d) & 5 & $300-560$ years & $1086 \mathrm{cal} \mathrm{AD}$ \\
\hline \multicolumn{4}{|c|}{ Mean Average 200 years IAT } & 5 & $465-680$ years & $1039 \mathrm{cal} \mathrm{AD}$ \\
\hline \multirow{4}{*}{$\begin{array}{l}500 \text { years IAt } \\
\text { M1e }\end{array}$} & 1 & 2270-1980 cal BC (M14e) & 1160-1280 cal AD (M12e) & 2 & $3205-3465$ years & $606 \mathrm{cal} \mathrm{AD}$ \\
\hline & 2 & 1270-1010 cal BC (M10e) & 1160-1280 cal AD (M1 1e) & 4 & $2245-2520$ years & 762 cal AD \\
\hline & 3 & 360-40 cal BC (M14e) & 1220-1390 cal AD (M5e) & 3 & $1315-1640$ years & $780 \mathrm{cal} \mathrm{AD}$ \\
\hline & 4 & 1950-1740 cal BC (M1e) & 1150-1280 cal AD (M10e) & 1 & 2920-3190 years & $491 \mathrm{cal} \mathrm{AD}$ \\
\hline \multicolumn{4}{|c|}{ Mean Average 500 years IAT } & 2.5 & 2420-2705 years & $670 \mathrm{cal} \mathrm{AD}$ \\
\hline
\end{tabular}

$(\mathrm{Ai} \leq 60 \%)$ and present low overall Combined Agreement Indices (Acomb) (Table 3). The exception is the 10 years IAt dataset in which all 20 simulated dates include the true event date (M1a run 1), which has a mean lifespan of $1242 \mathrm{cal}$ AD (the highest in the study and very close to the 1250 AD true event) and has only returned one low Ai. All sixteen simulated datasets specified with 50, 100, 200 and 500 years IAt fail the Ward and Wilson (1978) test. The four datasets specified to 50 years IAt (M1b runs $1-4$ ) and one specified to 100 years IAt (M1c run 1) have generated Combined date ranges, although these are all too early. The three remaining models associated with datasets specified to 100 years IAt (M1c runs 2-4) and all models specified with 200 and 500 years IAt datasets have failed to generate a Combined distribution at all.

Out-with the Combine models, all TS1 models except one present overall agreement indices which are above the $60 \%$ threshold (Table 4). The exception is associated with a uniform prior/no outlier modelling approach to a 50-year IAt dataset (M1b, run 4), which presents an Overall Agreement Index of 58.9\%. The number of individual dates with low Agreement Indices decreases with increasing IAt, and with models employing an exponential prior specification. All three uniform prior approaches generate higher numbers of individual low Agreement Index distributions than their exponential counterparts from 10 and 50 years IAt datasets. The uniform prior/no outlier approach contains the most low Agreement Index distributions in TS1, with a maximum of three presented from a 10 year IAt dataset (M1a run 3). Individual low Agreement Indices are limited to a single dates in all exponential prior modelling approaches (Table 4).

98\% of all TS1 models (118/120) have generated End Boundary HPD intervals which are accurate at $95 \%$ probability, and $85 \%(102 / 120)$ are also accurate at $68 \%$ probability (Table 5). Consistency of End Boundary accuracy at $68 \%$ probability is inversely proportional to dataset IAt; decreasing from $96 \%$ of models associated with datasets specified with 10 years IAt to $58 \%$ of models associated with datasets specified with 500 years IAt. Consistency of End Boundary accuracy also varies with model specification and a broad correlation with prior distribution 
Table 3 Combine distributions and agreement indices in TS1

\begin{tabular}{|c|c|c|c|c|c|c|c|c|}
\hline \multicolumn{2}{|l|}{ Dataset } & \multicolumn{4}{|c|}{ Combine date ranges/cal AD } & \multicolumn{3}{|c|}{ Agreement indices } \\
\hline Specified IAt and code & Run & $68 \%$ & $95 \%$ & median & mean & $x^{2}$ test & Acomb & $A i \leq 60 \%$ \\
\hline \multirow[t]{4}{*}{10 years $\mid A T ; M 1 a$} & 1 & $1230-1270$ & $1225-1270$ & 1245 & 1250 & Pass & 112 & 1 \\
\hline & 2 & $1220-1260$ & $1220-1265$ & 1245 & 1240 & Pass & 23.3 & 4 \\
\hline & 3 & $1220-1260$ & $1220-1265$ & 1245 & 1240 & Pass & 26.9 & 4 \\
\hline & 4 & $1220-1260$ & $1220-1265$ & 1245 & 1240 & Pass & 36.7 & 4 \\
\hline \multirow[t]{4}{*}{50 years $\mid A \tau ; M 1 b$} & 1 & $1175-1220$ & $1175-1220$ & 1190 & 1195 & Fail & 0.2 & 10 \\
\hline & 2 & $1175-1220$ & $1175-1220$ & 1190 & 1195 & Fail & 0.4 & 9 \\
\hline & 3 & $1210-1225$ & $1175-1225$ & 1215 & 1210 & Fail & 10.5 & 7 \\
\hline & 4 & $1175-1220$ & $1175-1220$ & 1190 & 1195 & Fail & 0.4 & 9 \\
\hline \multirow[t]{4}{*}{100 years IAt; M1C } & 1 & $1215-1225$ & $1180-1225$ & 1220 & 1220 & Fail & 2.3 & 8 \\
\hline & 2 & No range & No range & - & - & Fail & - & - \\
\hline & 3 & No range & No range & - & - & Fail & - & - \\
\hline & 4 & No range & No range & - & - & Fail & - & - \\
\hline \multirow[t]{4}{*}{200 years IAt; M1d } & 1 & No range & No range & - & - & Fail & - & - \\
\hline & 2 & No range & No range & - & - & Fail & - & - \\
\hline & 3 & No range & No range & - & - & Fail & - & - \\
\hline & 4 & No range & No range & - & - & Fail & - & - \\
\hline \multirow[t]{4}{*}{500 years $\mid \mathrm{A} \tau ;$ M1e } & 1 & No range & No range & - & - & Fail & - & - \\
\hline & 2 & No range & No range & - & - & Fail & - & - \\
\hline & 3 & No range & No range & - & - & Fail & - & - \\
\hline & 4 & No range & No range & - & - & Fail & - & - \\
\hline
\end{tabular}

Date ranges and median dates are rounded out to 5 years. Inaccurate date ranges (not including the $1250 \mathrm{AD}$ true event) are reported in bold emphasis

is evident; with $85-90 \%$ of models with exponential priors generating accurate End Boundary estimates at $68 \%$ probability, reducing to $70-85 \%$ of models with uniform prior distributions. Overall, the uniform prior/default Charcoal Outlier Model approach has generated the least consistently accurate estimates, with $70 \%(14 / 20)$ of all End Boundary HPD intervals associated with this specification including the true event date at $68 \%$ probability; ranging from $75 \%(3 / 4)$ of datasets specified to 10 years IAt, to $50 \%(2 / 4)$ of datasets specified to 100 years and 500 years IAt. The two End Boundary estimates in this study which are inaccurate at $95 \%$ probability are also associated with the same 500 years IAt dataset (M1e run 3) and with uniform prior/Charcoal Outlier Model approaches. The exponential prior/Charcoal Outlier Model approaches have generated the most consistently accurate End Boundary distributions in TS1; with this specification generating accurate End Boundaries at $68 \%$ probability from $90 \%$ of all datasets, and accurate End Boundaries at 95\% probability from all datasets (100\%). Notably, all models employing an exponential prior have generated accurate End Boundary HPD intervals at both $95 \%$ and $68 \%$ probability from all datasets specified with 10, 50 and 100 years IAt (Table 5).

Inaccurate End Boundary distributions at $68 \%$ probability in TS1 can be either earlier or later than the true event (Table 4). All inaccurate estimates generated by uniform prior models are late, including both of those which are inaccurate at $95 \%$ probability, whilst exponential prior models have generated End Boundary HPD intervals which are both too early and too late at $68 \%$ probability. The exponential prior/no outlier modelling approach to 50 years IAt and 200 years IAt datasets are the only two modelling-dataset combinations in TS1 which have generated End Boundary average medians that are earlier than the true event date of $1250 \mathrm{AD}$.

All Last HPD intervals generated in TS1 are accurate at 95\% probability (Table 6), although this would not have been the case had Last distributions been generated in runs 1 and 2. Last HPD interval accuracy at 68\% probability is inversely proportional to the specified dataset IAt; and falls sharply from $100 \%$ accuracy in models associated with 10 years and 50 years IAt datasets, to $58 \%$ accuracy in those generated from datasets specified to 500 years IAt. Most model specifications have generated accurate Last HPD intervals at $68 \%$ probability from $90 \%$ of all datasets. This is reduced to $80 \%$ for the uniform prior/default Charcoal Outlier Model approach, although this difference relates to one extra inaccurate date only.

End Boundary precision in TS1 is inversely proportional to specified dataset IAt for all modelling approaches (Table 7). Overall, End Boundary median 
Table 4 Last and End Boundary HPD intervals in TS1

\begin{tabular}{|c|c|c|c|c|c|c|c|c|c|c|}
\hline \multicolumn{2}{|l|}{ Dataset and model specification } & \multicolumn{3}{|c|}{$\begin{array}{l}\text { Last HPD intervals and medians/ } \\
\text { cal AD }\end{array}$} & \multicolumn{3}{|c|}{$\begin{array}{l}\text { End Boundary HPD intervals and } \\
\text { medians/cal } A D\end{array}$} & \multicolumn{3}{|c|}{ Agreement Indices } \\
\hline 10yrs IAt (M1a) & Run & $68 \%$ & $95 \%$ & Median & $68 \%$ & $95 \%$ & Median & Amodel & Aoverall & $A i \leq 60 \%$ \\
\hline \multirow[t]{4}{*}{ Exponential prior; No Outlier Model } & 1 & - & - & - & $1235-1275$ & $1230-1280$ & 1265 & 127.1 & 124 & 0 \\
\hline & 2 & - & - & - & $1240-1275$ & $1225-1280$ & 1255 & 80.2 & 69 & 1 \\
\hline & 3 & $1240-1270$ & $1225-1275$ & 1255 & $1240-1270$ & $1225-1280$ & 1255 & 100.9 & 91.5 & 0 \\
\hline & 4 & $1240-1270$ & $1225-1275$ & 1255 & $1240-1270$ & $1225-1275$ & 1255 & 107.8 & 97.6 & 0 \\
\hline \multirow[t]{4}{*}{ Exponential prior; 10-year Charcoal } & 1 & - & - & - & $1240-1280$ & $1235-1285$ & 1265 & 130.6 & 126.8 & 0 \\
\hline & 2 & - & - & - & $1245-1275$ & $1230-1285$ & 1260 & 81.3 & 71.7 & 1 \\
\hline & 3 & $1245-1275$ & $1230-1280$ & 1260 & $1245-1275$ & $1230-1280$ & 1260 & 101.8 & 94.1 & 0 \\
\hline & 4 & $1240-1270$ & $1230-1280$ & 1255 & $1240-1275$ & $1230-1280$ & 1255 & 108.3 & 99.9 & 0 \\
\hline \multirow[t]{4}{*}{ Exponential prior; Default Charcoal } & 1 & - & - & - & $1240-1280$ & $1235-1285$ & 1270 & 132.1 & 128.1 & 0 \\
\hline & 2 & - & - & - & $1245-1280$ & $1230-1285$ & 1260 & 84.7 & 75.6 & 1 \\
\hline & 3 & $1245-1275$ & $1230-1285$ & 1260 & $1245-1275$ & $1230-1285$ & 1260 & 104.7 & 98.2 & 0 \\
\hline & 4 & $1245-1275$ & $1230-1280$ & 1260 & $1245-1275$ & $1230-1285$ & 1260 & 111.2 & 104.3 & 0 \\
\hline \multirow[t]{4}{*}{ Uniform prior; No Outlier Model } & 1 & - & - & - & $1235-1280$ & $1230-1285$ & 1265 & 129.9 & 125.2 & 0 \\
\hline & 2 & - & - & - & $1240-1280$ & $1225-1285$ & 1260 & 86.4 & 61.8 & 2 \\
\hline & 3 & $1240-1275$ & $1225-1280$ & 1255 & $1240-1275$ & $1225-1285$ & 1260 & 94 & 72.7 & 3 \\
\hline & 4 & $1240-1270$ & $1225-1280$ & 1255 & $1240-1275$ & $1225-1280$ & 1255 & 97.8 & 78 & 2 \\
\hline \multirow[t]{4}{*}{ Uniform prior; 10-year Charcoal } & 1 & - & - & - & $1240-1285$ & $1235-1290$ & 1270 & 131.1 & 126.4 & 0 \\
\hline & 2 & - & - & - & $1245-1280$ & $1230-1290$ & 1260 & 87.1 & 67.9 & 2 \\
\hline & 3 & $1240-1275$ & $1230-1285$ & 1260 & $1240-1275$ & $1230-1285$ & 1260 & 91.3 & 76 & 2 \\
\hline & 4 & $1240-1275$ & $1225-1280$ & 1255 & $1240-1275$ & $1225-1285$ & 1260 & 97.3 & 83 & 2 \\
\hline \multirow[t]{4}{*}{ Uniform prior; Default Charcoal } & 1 & - & - & - & $1255-1285$ & $1235-1290$ & 1270 & 134 & 129.1 & 0 \\
\hline & 2 & - & - & - & $1245-1285$ & $1230-1295$ & 1265 & 90.8 & 75.4 & 1 \\
\hline & 3 & $1245-1280$ & $1230-1290$ & 1265 & $1245-1280$ & $1230-1295$ & 1265 & 100.1 & 89.2 & 0 \\
\hline & 4 & $1245-1275$ & $1230-1285$ & 1260 & $1245-1280$ & $1230-1290$ & 1260 & 105.8 & 95.3 & 0 \\
\hline 50yrs IAt (M1b) & Run & $68 \%$ & $95 \%$ & Median & $68 \%$ & $95 \%$ & Median & Amodel & Aoverall & $A i \leq 60 \%$ \\
\hline \multirow[t]{4}{*}{ Exponential prior; No Outlier Model } & 1 & - & - & - & $1240-1275$ & $1230-1285$ & 1255 & 92.5 & 91 & 0 \\
\hline & 2 & - & - & - & $1230-1265$ & $1225-1285$ & 1250 & 73.6 & 73.7 & 1 \\
\hline & 3 & $1220-1250$ & $1220-1270$ & 1240 & $1220-1255$ & $1220-1275$ & 1240 & 94.5 & 91.2 & 0 \\
\hline & 4 & $1235-1265$ & $1225-1275$ & 1250 & $1235-1270$ & $1225-1280$ & 1250 & 115.2 & 114.3 & 0 \\
\hline \multirow[t]{4}{*}{ Exponential prior; 50-year Charcoal } & 1 & - & - & - & $1245-1285$ & $1235-1300$ & 1265 & 92.9 & 91.2 & 0 \\
\hline & 2 & - & - & - & $1235-1275$ & $1230-1295$ & 1260 & 78.5 & 78 & 1 \\
\hline & 3 & $1225-1260$ & $1220-1280$ & 1245 & $1225-1260$ & $1220-1280$ & 1245 & 96 & 93.8 & 0 \\
\hline & 4 & $1240-1270$ & $1230-1285$ & 1255 & $1240-1275$ & $1230-1290$ & 1260 & 113.8 & 112.9 & 0 \\
\hline \multirow[t]{4}{*}{ Exponential prior; Default Charcoal } & 1 & - & - & - & $1245-1285$ & $1235-1300$ & 1265 & 93.2 & 91.7 & 0 \\
\hline & 2 & - & - & - & $1240-1280$ & $1230-1300$ & 1260 & 80.2 & 79.8 & 1 \\
\hline & 3 & $1225-1260$ & $1220-1280$ & 1245 & $1225-1265$ & $1220-1280$ & 1250 & 96.5 & 94.5 & 0 \\
\hline & 4 & $1240-1275$ & $1230-1290$ & 1260 & $1240-1280$ & $1230-1290$ & 1260 & 114.2 & 113.5 & 0 \\
\hline \multirow{4}{*}{ Uniform prior; No Outlier Model } & 1 & - & - & - & $1255-1290$ & $1235-1305$ & 1270 & 89.7 & 88.5 & 0 \\
\hline & 2 & - & - & - & $1245-1290$ & $1230-1305$ & 1270 & 76.5 & 75.2 & 2 \\
\hline & 3 & $1225-1260$ & $1220-1275$ & 1245 & $1225-1265$ & $1220-1285$ & 1250 & 83.6 & 79.2 & 2 \\
\hline & 4 & $1240-1270$ & $1230-1280$ & 1255 & $1240-1275$ & $1230-1290$ & 1260 & 57.7 & 58.9 & 1 \\
\hline \multirow[t]{4}{*}{ Uniform prior; 50-year Charcoal } & 1 & - & - & - & $1255-1295$ & $1235-1315$ & 1275 & 89.1 & 87.8 & 0 \\
\hline & 2 & - & - & - & $1250-1300$ & $1235-1320$ & 1275 & 81.6 & 80.6 & 2 \\
\hline & 3 & $1230-1270$ & $1220-1285$ & 1250 & $1230-1270$ & $1220-1290$ & 1255 & 88.8 & 85.5 & 2 \\
\hline & 4 & $1245-1280$ & $1230-1295$ & 1265 & $1245-1285$ & $1230-1305$ & 1265 & 93 & 92.1 & 1 \\
\hline Uniform prior; Default Charcoal & 1 & - & - & - & $1255-1295$ & $1240-1320$ & 1280 & 90.6 & 89.4 & 0 \\
\hline
\end{tabular}


Table 4 (continued)

\begin{tabular}{|c|c|c|c|c|c|c|c|c|c|c|}
\hline 50yrs IAt (M1b) & Run & $68 \%$ & $95 \%$ & Median & $68 \%$ & $95 \%$ & Median & Amodel & Aoverall & $A i \leq 60 \%$ \\
\hline & 2 & - & - & - & $1250-1300$ & $1235-1320$ & 1280 & 84.3 & 83.6 & 2 \\
\hline & 3 & $1230-1270$ & $1220-1285$ & 1250 & $1230-1270$ & $1225-1290$ & 1255 & 88.6 & 85.5 & 2 \\
\hline & 4 & $1245-1285$ & $1230-1305$ & 1265 & $1250-1290$ & $1235-1315$ & 1270 & 102.2 & 101.5 & 1 \\
\hline 100yrs IAt (M1c) & Run & $68 \%$ & $95 \%$ & Median & $68 \%$ & $95 \%$ & Median & Amodel & Aoverall & $A i \leq 60 \%$ \\
\hline \multirow[t]{4}{*}{ Exponential prior; No Outlier Model } & 1 & - & - & - & $1240-1275$ & $1230-1280$ & 1255 & 99.1 & 98 & 0 \\
\hline & 2 & - & - & - & $1235-1270$ & $1225-1285$ & 1255 & 108.8 & 108.5 & 0 \\
\hline & 3 & $1240-1270$ & $1225-1275$ & 1255 & $1245-1275$ & $1225-1290$ & 1260 & 107.6 & 107.4 & 0 \\
\hline & 4 & $1230-1265$ & $1220-1275$ & 1245 & $1235-1270$ & $1225-1280$ & 1250 & 98.2 & 97 & 0 \\
\hline \multirow[t]{4}{*}{ Exponential prior; 100-year Charcoal } & | 1 & - & - & - & $1245-1280$ & $1230-1295$ & 1265 & 101.1 & 99.7 & 0 \\
\hline & 2 & - & - & - & $1240-1280$ & $1230-1300$ & 1260 & 108.9 & 108.4 & 0 \\
\hline & 3 & $1245-1275$ & $1225-1295$ & 1260 & $1245-1285$ & $1230-1305$ & 1265 & 106.7 & 106.6 & 0 \\
\hline & 4 & $1235-1275$ & $1225-1290$ & 1260 & $1240-1280$ & $1225-1300$ & 1260 & 97.6 & 96.4 & 0 \\
\hline \multirow[t]{4}{*}{ Exponential prior; Default Charcoal } & 1 & - & - & - & $1245-1280$ & $1235-1290$ & 1265 & 100.9 & 99.8 & 0 \\
\hline & 2 & - & - & - & $1245-1280$ & $1230-1300$ & 1265 & 108.9 & 108.8 & 0 \\
\hline & 3 & $1245-1275$ & $1225-1295$ & 1260 & $1245-1285$ & $1230-1305$ & 1265 & 106.8 & 106.6 & 0 \\
\hline & 4 & $1240-1275$ & $1225-1295$ & 1260 & $1240-1280$ & $1225-1300$ & 1265 & 97.7 & 96.7 & 0 \\
\hline \multirow[t]{4}{*}{ Uniform prior; No Outlier Model } & 1 & - & - & - & $1240-1285$ & $1225-1295$ & 1260 & 82.6 & 70.4 & 2 \\
\hline & 2 & - & - & - & $1250-1290$ & $1235-1320$ & 1275 & 69.3 & 70 & 1 \\
\hline & 3 & $1250-1275$ & $1240-1280$ & 1265 & $1255-1315$ & $1240-1385$ & 1285 & 94.8 & 94.8 & 0 \\
\hline & 4 & $1245-1275$ & $1230-1280$ & 1260 & $1250-1290$ & $1230-1315$ & 1270 & 85.2 & 85 & 1 \\
\hline \multirow[t]{4}{*}{ Uniform prior; 100-year Charcoal } & 1 & - & - & - & $1250-1290$ & $1235-1310$ & 1270 & 96.5 & 92.9 & 0 \\
\hline & 2 & - & - & - & $1245-1295$ & $1230-1330$ & 1275 & 107.9 & 107.2 & 0 \\
\hline & 3 & $1250-1290$ & $1235-1320$ & 1270 & $1250-1295$ & $1230-1325$ & 1275 & 104.3 & 103.8 & 0 \\
\hline & 4 & $1245-1290$ & $1230-1320$ & 1270 & $1250-1300$ & $1230-1335$ & 1275 & 93.3 & 92.3 & 0 \\
\hline \multirow[t]{4}{*}{ Uniform prior; Default Charcoal } & 1 & - & - & - & $1250-1290$ & $1235-1310$ & 1270 & 96.3 & 92.5 & 0 \\
\hline & 2 & - & - & - & $1250-1300$ & $1235-1335$ & 1280 & 108.1 & 107.8 & 0 \\
\hline & 3 & $1255-1305$ & $1240-1345$ & 1285 & $1255-1310$ & $1240-1350$ & 1285 & 105 & 104.8 & 0 \\
\hline & 4 & $1250-1300$ & $1230-1330$ & 1280 & $1255-1310$ & $1235-1345$ & 1285 & 95 & 94.1 & 0 \\
\hline 200yrs IAt (M1d) & Run & $68 \%$ & $95 \%$ & Median & $68 \%$ & $95 \%$ & Median & Amodel & Aoverall & $A i \leq 60 \%$ \\
\hline \multirow[t]{4}{*}{ Exponential prior; no Outlier Model } & 1 & - & - & - & $1220-1270$ & $1190-1300$ & 1245 & 96.5 & 96.7 & 0 \\
\hline & 2 & - & - & - & $1175-1220$ & 1155-1250 & 1200 & 97.8 & 95.6 & 0 \\
\hline & 3 & $1220-1260$ & $1200-1275$ & 1235 & $1225-1270$ & $1200-1300$ & 1250 & 98.3 & 97.3 & 0 \\
\hline & 4 & $1185-1230$ & $1170-1250$ & 1210 & $1190-1235$ & $51175-1260$ & 1215 & 83.9 & 84.3 & 1 \\
\hline \multirow[t]{4}{*}{ Exponential prior; 200-year Charcoal } & | 1 & - & - & - & $1225-1285$ & $1195-1330$ & 1255 & 97.5 & 97 & 0 \\
\hline & 2 & - & - & - & $1180-1240$ & 1160-1285 & 1210 & 97.9 & 95.7 & 0 \\
\hline & 3 & $1225-1275$ & $1200-1320$ & 1255 & $1230-1290$ & $1210-1335$ & 1265 & 98.9 & 98.3 & 0 \\
\hline & 4 & $1200-1260$ & $1180-1280$ & 1225 & $1200-1260$ & $1185-1285$ & 1235 & 89.8 & 89.8 & 1 \\
\hline \multirow[t]{4}{*}{ Exponential prior; default Charcoal } & 1 & - & - & - & $1230-1295$ & $1200-1340$ & 1265 & 97.5 & 97.6 & 0 \\
\hline & 2 & - & - & - & $1185-1240$ & o $1165-1285$ & 1215 & 98.2 & 96.3 & 0 \\
\hline & 3 & $1225-1270$ & $1210-1300$ & 1250 & $1230-1285$ & $1215-1320$ & 1260 & 99.2 & 98.8 & 0 \\
\hline & 4 & $1195-1255$ & $1180-1285$ & 1230 & $1200-1260$ & $1180-1290$ & 1235 & 90 & 90.1 & 1 \\
\hline \multirow[t]{4}{*}{ Uniform prior; No Outlier Model } & 1 & - & - & - & $1230-1300$ & $1205-1370$ & 1270 & 97.9 & 98.1 & 0 \\
\hline & 2 & - & - & - & $1190-1250$ & $1170-1290$ & 1225 & 91.6 & 90.3 & 0 \\
\hline & 3 & $1230-1270$ & $1215-1280$ & 1250 & $1235-1300$ & $1215-1365$ & 1270 & 102.3 & 102 & 0 \\
\hline & 4 & $1205-1255$ & $1180-1270$ & 1225 & $1210-1270$ & $1180-1300$ & 1240 & 76.4 & 76 & 1 \\
\hline \multirow[t]{3}{*}{ Uniform prior; 200-year Charcoal } & 1 & - & - & - & $1245-1350$ & $1220-1450$ & 1305 & 97.4 & 97.3 & 0 \\
\hline & 2 & - & - & - & $1190-1265$ & $1170-1315$ & 1235 & 95.8 & 94.5 & 0 \\
\hline & 3 & $1235-1300$ & $1215-1375$ & 1270 & $1245-1335$ & $1225-1420$ & 1295 & 100 & 99.6 & 0 \\
\hline
\end{tabular}


Table 4 (continued)

\begin{tabular}{|c|c|c|c|c|c|c|c|c|c|c|}
\hline 200yrs IAt (M1d) & Run & $68 \%$ & $95 \%$ & Median & $68 \%$ & $95 \%$ & Media & Amodel & Aoverall & $A i \leq 60 \%$ \\
\hline & 4 & $1220-1280$ & $1190-1320$ & 1250 & $1225-1295$ & $1195-1345$ & 1265 & 91.7 & 92.1 & 0 \\
\hline \multirow[t]{2}{*}{ Uniform prior; default Charcoal } & 1 & - & - & - & $1250-1355$ & $1225-1425$ & 1305 & 98.3 & 98.3 & 0 \\
\hline & 2 & - & - & - & $1205-1275$ & $1175-1335$ & 1240 & 92.4 & 91.4 & 0 \\
\hline 500yrs IAt (M1e) & Run & $68 \%$ & $95 \%$ & Median & $68 \%$ & $95 \%$ & Media & $n$ Amodel & Aoverall & $A i \leq 60 \%$ \\
\hline \multirow[t]{4}{*}{ exponential prior; no Outlier Model } & 1 & - & & - & $1225-1300$ & $1190-1370$ & 1265 & 98 & 97.4 & 0 \\
\hline & 2 & - & & - & $1225-1290$ & $1195-1345$ & 1260 & 99.2 & 98.8 & 0 \\
\hline & 3 & $1230-1295$ & $1225-1300$ & 1270 & $1255-1325$ & $1225-1375$ & 1290 & 97.4 & 97.2 & 0 \\
\hline & 4 & $1160-1230$ & $1120-1265$ & 1190 & $1170-1275$ & $1120-1355$ & 1225 & 95.4 & 95.9 & 0 \\
\hline \multirow[t]{4}{*}{ Exponential prior; 500-year Charcoal } & & - & - & - & $1235-1325$ & $1200-1405$ & 1285 & 98.4 & 97.9 & 0 \\
\hline & 2 & - & - & - & $1230-1310$ & $1205-1375$ & 1275 & 99 & 98.7 & 0 \\
\hline & 3 & $1255-1335$ & $1225-1405$ & 1295 & $1270-1365$ & $1235-1440$ & 1320 & 98.8 & 98.7 & 0 \\
\hline & 4 & $1165-1255$ & $1110-1350$ & 1210 & $1180-1300$ & $1135-1420$ & 1250 & 96.6 & 97.2 & 0 \\
\hline \multirow[t]{4}{*}{ Exponential prior; default Charcoal } & 1 & - & - & - & $1235-1325$ & $1200-1400$ & 1280 & 98.1 & 97.8 & 0 \\
\hline & 2 & - & - & - & $1230-1315$ & $1205-1395$ & 1280 & 99 & 98.7 & 0 \\
\hline & 3 & $1245-1335$ & $1225-1415$ & 1295 & $1270-1365$ & $1235-1450$ & 1320 & 98.6 & 98.6 & 0 \\
\hline & 4 & $1165-1260$ & $1115-1345$ & 1215 & $1180-1305$ & $1140-1420$ & 1250 & 96.6 & 97.1 & 0 \\
\hline \multirow[t]{4}{*}{ Uniform prior; No Outlier Model } & 1 & - & - & - & $1230-1430$ & $1205-1740$ & 1345 & 99.2 & 99.1 & 0 \\
\hline & 2 & - & - & - & $1235-1380$ & $1205-1605$ & 1315 & 96.9 & 96.8 & 0 \\
\hline & 3 & $1260-1295$ & $1225-1305$ & 1275 & $1265-1370$ & $1230-1500$ & 1320 & 98 & 98.1 & 0 \\
\hline & 4 & $1170-1255$ & $1150-1275$ & 1210 & $1185-1385$ & $1150-1665$ & 1300 & 98.8 & 99 & 0 \\
\hline \multirow[t]{4}{*}{ Uniform prior; 500-year Charcoal } & 1 & - & - & - & $1260-1585$ & $1220-1870$ & 1450 & 99.6 & 99.5 & 0 \\
\hline & 2 & - & - & - & $1270-1460$ & $1240-1685$ & 1375 & 98.1 & 98 & 0 \\
\hline & 3 & $1270-1405$ & $1235-1535$ & 1350 & $1300-1455$ & $1265-1605$ & 1390 & 99.6 & 99.7 & 0 \\
\hline & 4 & $1170-1285$ & $1145-1490$ & 1245 & $1215-1495$ & $1170-1795$ & 1375 & 99.8 & 99.8 & 0 \\
\hline \multirow[t]{4}{*}{ Uniform prior; default Charcoal } & 1 & - & - & - & $1245-1470$ & $1215-1790$ & 1375 & 99.4 & 99.4 & 0 \\
\hline & 2 & - & - & - & $1265-1485$ & $1230-1685$ & 1405 & 97.5 & 97.6 & 0 \\
\hline & 3 & $1270-1410$ & $1235-1535$ & 1350 & $1295-1460$ & $1265-1605$ & 1395 & 99.5 & 99.5 & 0 \\
\hline & 4 & $1165-1350$ & $1140-1625$ & 1260 & $1220-1540$ & $1175-1905$ & 1405 & 99.6 & 99.8 & 0 \\
\hline
\end{tabular}

HPD intervals and median dates are rounded out to 5 years. Inaccurate HPD intervals (not including the 1250 AD true event date) and agreement indices less than $60 \%$ are reported in bold emphasis

Table 5 Accuracy of End Boundary HPD intervals in TS1 (summarised from Table 4)

\begin{tabular}{|c|c|c|c|c|c|c|c|c|}
\hline \multirow[t]{3}{*}{ Specified dataset IAT } & \multicolumn{8}{|c|}{ Accuracy of End Boundary HPD intervals } \\
\hline & \multirow{2}{*}{$\begin{array}{l}\text { Exp. prior; } \\
\text { no Outlier } \\
68 \%(95 \%)\end{array}$} & \multirow{2}{*}{$\begin{array}{l}\text { Exp. prior; } \\
\text { modified } \\
\text { Charcoal } \\
68 \%(95 \%)\end{array}$} & \multirow{2}{*}{ 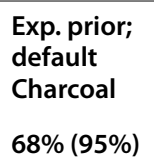 } & \multirow{2}{*}{$\begin{array}{l}\text { Uniform prior; } \\
\text { no Outlier } \\
68 \%(95 \%)\end{array}$} & \multirow{2}{*}{$\begin{array}{l}\text { Uniform prior; } \\
\text { modified } \\
\text { Charcoal } \\
68 \%(95 \%)\end{array}$} & \multirow{2}{*}{$\begin{array}{l}\begin{array}{l}\text { Uniform prior; } \\
\text { default Charcoal }\end{array} \\
68 \%(95 \%)\end{array}$} & \multicolumn{2}{|c|}{ All models } \\
\hline & & & & & & & $68 \%$ & $95 \%$ \\
\hline 10 years & $4 / 4(4 / 4)$ & $4 / 4(4 / 4)$ & $4 / 4(4 / 4)$ & $4 / 4(4 / 4)$ & $4 / 4(4 / 4)$ & $3 / 4(4 / 4)$ & $96 \%$ & $100 \%$ \\
\hline 50 years & $4 / 4(4 / 4)$ & $4 / 4(4 / 4)$ & $4 / 4(4 / 4)$ & $3 / 4(4 / 4)$ & $3 / 4(4 / 4)$ & $3 / 4(4 / 4)$ & $88 \%$ & $100 \%$ \\
\hline 100 years & $4 / 4(4 / 4)$ & $4 / 4(4 / 4)$ & $4 / 4(4 / 4)$ & $3 / 4(4 / 4)$ & $4 / 4(4 / 4)$ & $2 / 4(4 / 4)$ & $88 \%$ & $100 \%$ \\
\hline 200 years & $2 / 4(4 / 4)$ & $3 / 4(4 / 4)$ & $3 / 4(4 / 4)$ & $4 / 4(4 / 4)$ & $4 / 4(4 / 4)$ & $4 / 4(4 / 4)$ & $83 \%$ & $100 \%$ \\
\hline 500 years & $3 / 4(4 / 4)$ & $3 / 4(4 / 4)$ & $3 / 4(4 / 4)$ & $3 / 4(4 / 4)$ & $1 / 4(3 / 4)$ & $2 / 4(3 / 4)$ & $58 \%$ & $92 \%$ \\
\hline All Datasets & $85 \%(100 \%)$ & $90 \%$ (100\%) & $90 \%(100 \%)$ & $85 \%(100 \%)$ & $80 \%$ (95\%) & $70 \%$ (95\%) & $85 \%$ & $98 \%$ \\
\hline
\end{tabular}

age is also inversely proportional to dataset IAt across the study, although that trend is much clearer in models with uniform priors (Table 8). End Boundary precision and median age also vary with model specification; such that the imposition of an exponential prior distribution generally increases relative End Boundary precision and 
Table 6 Accuracy of Last HPD intervals in TS1 (summarised from Table 4)

\begin{tabular}{|c|c|c|c|c|c|c|c|c|}
\hline \multirow{3}{*}{$\begin{array}{l}\text { Specified } \\
\text { dataset IAt }\end{array}$} & \multicolumn{8}{|c|}{ Accuracy of Last HPD intervals } \\
\hline & \multirow{2}{*}{$\begin{array}{l}\text { Exp. prior; } \\
\text { no Outlier } \\
68 \%(95 \%)\end{array}$} & \multirow{2}{*}{$\begin{array}{l}\text { Exp. prior; } \\
\text { modified } \\
\text { Charcoal } \\
68 \%(95 \%)\end{array}$} & \multicolumn{2}{|c|}{$\begin{array}{l}\text { Exp. prior; Uniform prior; } \\
\text { default Charcoal no Outlier }\end{array}$} & \multirow{2}{*}{$\begin{array}{l}\text { Uniform prior; } \\
\text { modified } \\
\text { Charcoal } \\
68 \%(95 \%)\end{array}$} & \multirow{2}{*}{$\begin{array}{l}\begin{array}{l}\text { Uniform prior; } \\
\text { default Charcoal }\end{array} \\
68 \%(95 \%)\end{array}$} & \multicolumn{2}{|c|}{ All models } \\
\hline & & & $68 \%(95 \%)$ & $68 \%(95 \%)$ & & & $68 \%$ & $95 \%$ \\
\hline 10 years & $2 / 2(2 / 2)$ & $2 / 2(2 / 2)$ & $2 / 2(2 / 2)$ & $2 / 2(2 / 2)$ & $2 / 2(2 / 2)$ & $2 / 2(2 / 2)$ & $100 \%$ & $100 \%$ \\
\hline 50 years & $2 / 2(2 / 2)$ & $2 / 2(2 / 2)$ & $2 / 2(2 / 2)$ & $2 / 2(2 / 2)$ & $2 / 2(2 / 2)$ & $2 / 2(2 / 2)$ & $100 \%$ & $100 \%$ \\
\hline 100 years & $2 / 2(2 / 2)$ & $2 / 2(2 / 2)$ & $2 / 2(2 / 2)$ & $2 / 2(2 / 2)$ & $2 / 2(2 / 2)$ & $1 / 2(2 / 2)$ & $92 \%$ & $100 \%$ \\
\hline 200 years & $1 / 2(2 / 2)$ & $2 / 2(2 / 2)$ & $2 / 2(2 / 2)$ & $2 / 2(2 / 2)$ & $2 / 2(2 / 2)$ & $2 / 2(2 / 2)$ & $92 \%$ & $100 \%$ \\
\hline 500 years & $2 / 2(2 / 2)$ & $1 / 2(2 / 2)$ & $1 / 2(2 / 2)$ & $1 / 2(2 / 2)$ & $1 / 2(2 / 2)$ & $1 / 2(2 / 2)$ & $58 \%$ & $100 \%$ \\
\hline All Datasets & $90 \%(100 \%)$ & $90 \%(100 \%)$ & $90 \%(100 \%)$ & $90 \%(100 \%)$ & $90 \%(100 \%)$ & $80 \%(100 \%)$ & $88 \%$ & $100 \%$ \\
\hline
\end{tabular}

Table 7 Precision of End Boundary HPD intervals in TS1 (summarised from Table 4)

\begin{tabular}{|c|c|c|c|c|c|c|}
\hline \multirow[t]{3}{*}{ Specified dataset IAT } & \multicolumn{6}{|c|}{ Average precision of End Boundary HPD intervals/years } \\
\hline & $\begin{array}{l}\text { Exp. prior; no } \\
\text { Outlier Model }\end{array}$ & $\begin{array}{l}\text { Exp. prior; } \\
\text { modified Charcoal }\end{array}$ & $\begin{array}{l}\text { Exp. prior; default } \\
\text { Charcoal }\end{array}$ & $\begin{array}{l}\text { Uniform prior; } \\
\text { no Outlier }\end{array}$ & $\begin{array}{l}\text { Uniform prior; } \\
\text { mod. Charcoal }\end{array}$ & $\begin{array}{l}\text { Uniform } \\
\text { prior; def. } \\
\text { Charcoal }\end{array}$ \\
\hline & $68 \%(95 \%)$ & $68 \%(95 \%)$ & $68 \%(95 \%)$ & $68 \%(95 \%)$ & $68 \%(95 \%)$ & $68 \%(95 \%)$ \\
\hline 10 years & $34(53)$ & $34(53)$ & $34(51)$ & $39(58)$ & $38(63)$ & $35(65)$ \\
\hline 50 years & $35(56)$ & $38(63)$ & $40(64)$ & $39(68)$ & $43(78)$ & $43(78)$ \\
\hline 100 years & $34(58)$ & $39(71)$ & $38(69)$ & $46(96)$ & $46(94)$ & $50(99)$ \\
\hline 200 years & $46(96)$ & $58(120)$ & $58(116)$ & $66(153)$ & $86(154)$ & $89(178)$ \\
\hline 500 years & 79 (179) & $96(216)$ & 99 (221) & $163(430)$ & $238(515)$ & $233(525)$ \\
\hline
\end{tabular}

Table 8 Average End Boundary median values in TS1 (summarised from Table 4)

\begin{tabular}{lllllll}
\hline Specified dataset IAT & \multicolumn{2}{l}{ Average median of End Boundary HPD intervals/cal AD } \\
\cline { 2 - 7 } & $\begin{array}{l}\text { Exp. prior; no } \\
\text { Outlier Model }\end{array}$ & $\begin{array}{l}\text { Exp. prior; } \\
\text { modified } \\
\text { Charcoal }\end{array}$ & $\begin{array}{l}\text { Exp. prior; } \\
\text { default Charcoal }\end{array}$ & $\begin{array}{l}\text { Uniform prior; } \\
\text { no Outlier }\end{array}$ & $\begin{array}{l}\text { Uniform prior; } \\
\text { modified Charcoal }\end{array}$ & $\begin{array}{l}\text { Uniform } \\
\text { prior; default } \\
\text { Charcoal }\end{array}$ \\
\hline 10 years & 1258 & 1260 & 1263 & 1260 & 1258 & 1263 \\
50 years & 1249 & 1258 & 1259 & 1263 & 1268 & 1271 \\
100 years & 1255 & 1263 & 1265 & 1273 & 1274 & 1280 \\
200 years & 1241 & 1258 & 1256 & 1266 & 1290 & 1290 \\
500 years & 1260 & 1283 & 1283 & 1320 & 1398 & 1395 \\
\hline
\end{tabular}

median age (whether or not a Charcoal Outlier Model is also used), whilst the introduction of a Charcoal Outlier Model generally decreases relative End Boundary precision and median age (whatever prior distribution is employed) (Tables 7 and 8). Decreasing the Charcoal Outlier Model time-constant has generally increased End Boundary median age in lower IAt TS1 datasets (whatever the priors) and slightly increased End Boundary precision in models associated with uniform priors (Tables 7 and 8). These effects are cumulative; such that variation in model specification has a much more significant on End Boundary precision and median age when associated with higher IAt datasets.

Last HPD interval precision in TS1 is also inversely proportional to dataset IAt for all modelling approaches (Table 9). Last precision also varies according to model 
Table 9 Precision of Last HPD intervals in TS1 (summarised from Table 4)

\begin{tabular}{|c|c|c|c|c|c|c|}
\hline \multirow[t]{3}{*}{ Specified dataset IAT } & \multicolumn{6}{|c|}{ Average precision of Last HPD intervals/years } \\
\hline & $\begin{array}{l}\text { Exp. prior; no } \\
\text { Outlier Model }\end{array}$ & $\begin{array}{l}\text { Exp. prior; } \\
\text { modified Charcoal }\end{array}$ & $\begin{array}{l}\text { Exp. prior; } \\
\text { default Charcoal }\end{array}$ & $\begin{array}{l}\text { Uniform prior; } \\
\text { no Outlier }\end{array}$ & $\begin{array}{l}\text { Uniform prior; } \\
\text { mod. Charcoal }\end{array}$ & $\begin{array}{l}\text { Uniform } \\
\text { prior; default } \\
\text { Charcoal }\end{array}$ \\
\hline & $68 \%(95 \%)$ & $68 \%(95 \%)$ & $68 \%(95 \%)$ & $68 \%(95 \%)$ & $68 \%(95 \%)$ & $68 \%(95 \%)$ \\
\hline 10 years & $30(50)$ & $30(50)$ & $30(53)$ & $33(55)$ & $35(55)$ & $33(58)$ \\
\hline 50 years & $30(50)$ & $33(58)$ & $35(60)$ & $33(58)$ & $38(65)$ & $40(70)$ \\
\hline 100 years & $33(53)$ & $35(68)$ & $33(70)$ & $28(45)$ & $43(88)$ & $50(103)$ \\
\hline 200 years & $43(78)$ & $55(110)$ & $53(98)$ & $45(78)$ & $63(145)$ & $58(110)$ \\
\hline 500 years & $68(110)$ & $80(210)$ & $93(210)$ & $60(103)$ & 125 (323) & 163 (393) \\
\hline
\end{tabular}

Table 10 Average Last median values in TS1 (summarised from Table 4)

\begin{tabular}{lllllll}
\hline Specified dataset IAT & \multicolumn{2}{l}{ Average medians of Last HPD intervals/cal AD } \\
\cline { 2 - 7 } & $\begin{array}{l}\text { Exp. prior; no } \\
\text { Outlier Model }\end{array}$ & $\begin{array}{l}\text { Exp. prior; } \\
\text { modified } \\
\text { Charcoal }\end{array}$ & $\begin{array}{l}\text { Exp. prior; } \\
\text { default Charcoal }\end{array}$ & $\begin{array}{l}\text { Uniform prior; } \\
\text { no Outlier }\end{array}$ & $\begin{array}{l}\text { Uniform prior; } \\
\text { modified Charcoal }\end{array}$ & $\begin{array}{l}\text { Uniform } \\
\text { prior; default } \\
\text { Charcoal }\end{array}$ \\
\hline 10 years & 1255 & 1258 & 1260 & 1255 & 1258 & 1263 \\
50 years & 1245 & 1250 & 1253 & 1250 & 1258 & 1258 \\
100 years & 1250 & 1260 & 1260 & 1263 & 1270 & 1283 \\
200 years & 1223 & 1240 & 1240 & 1238 & 1260 & 1258 \\
500 years & 1230 & 1253 & 1245 & 1243 & 1298 & 1305 \\
\hline
\end{tabular}

specification: decreasing on association with a Charcoal Outlier Model (whichever prior distribution is used) and increasing on association with exponential priors and lower IAt datasets. In general, these two factors have a cumulative effect, although the Last distributions generated by the uniform prior/no outlier model approach display a lower decrease in precision with increased dataset IAt than other modelling approaches and are thereby associated much greater comparative precision at higher datasets IAts. Modifying outlier model time-constant has no clear effect on Last precision and there is no clear relationship between Last median ages and dataset IAt. Importantly, all model-dataset combinations in TS1 have generated Last distributions which are more precise and have earlier median values than their corresponding End Boundaries, and this contrast is also more significant with increasing dataset IAt (Tables 7, 8, 9 and 10). 37\% $(11 / 30)$ of the model-dataset combinations in TS1 have returned Last distributions with average medians of 1250 AD or earlier (Table 10).

\section{Theoretical Study 2 (TS2)}

Most TS2 datasets present convincingly exponential distributions, and 91\% (41/45) contain at least one simulated date which includes the true event (Table 11). Increased
IAt specification in this study has generally resulted in datasets with earlier latest dates, a decreased number of dates which include the true event, increased age ranges, increased age range variation, earlier earliest dates, and earlier mean lifespans. No latest dates in TS2 are later than the true event at $95 \%$ probability. Decreased dataset size has also resulted in earlier latest dates, however, and at very high IAt this can result in no true dates at all. There is no clear correlation between dataset size and percentage of true dates or mean lifespans for a given IAT across the study, but earliest dates tend to decrease in age with decreased dataset size and thereby age range and age variation also decrease (since this is mostly driven by the early dates) (Fig. 4a-c).

The inverse correlation between dataset IAt and the age of the latest date is exaggerated by dataset size. There is a strong correlation in TS2 between the specified IAt and the number of accurate dates in each dataset, with $94-98 \%$ of all determinations including the true event date at $95 \%$ confidence in datasets specified with 10 years IAt, and $67 \%(6 / 9)$ of these 10 years IAt datasets are completely dominated by such accurate simulated dates in all three dataset sizes. At the other end of the IAt spectrum, only $6-13 \%$ of datasets specified with 500 years IAt are dates which include the true event at 
Table 11 Selected features of the TS2 datasets

\begin{tabular}{|c|c|c|c|c|c|c|c|c|}
\hline Dataset IAT & Dataset Size & Dataset Code & Run & $\begin{array}{l}\text { Earliest date } \\
(95 \% \\
\text { confidence) }\end{array}$ & $\begin{array}{l}\text { Latest date } \\
(95 \% \\
\text { confidence })\end{array}$ & $\begin{array}{l}\text { Accurate } \\
\text { dates }(95 \%)\end{array}$ & $\begin{array}{l}\text { Age range } \\
(95 \% \\
\text { probability) }\end{array}$ & $\begin{array}{l}\text { Mean } \\
\text { lifespan }\end{array}$ \\
\hline \multirow[t]{12}{*}{10 Years } & 15 dates & MR1a & 1 & $\begin{array}{l}\text { 1040-1230 } \\
\text { (MR15a) }\end{array}$ & $\begin{array}{l}\text { 1220-1300 } \\
\text { (MR11a) }\end{array}$ & 14 & $15-230$ years & $1226 \mathrm{cal} \mathrm{AD}$ \\
\hline & & & 2 & $\begin{array}{l}1040-1270 \\
\text { (MR13a) }\end{array}$ & $\begin{array}{l}\text { 1220-1380 } \\
\text { (MR4a and M14a) }\end{array}$ & 15 & $10-240$ years & $1234 \mathrm{cal} \mathrm{AD}$ \\
\hline & & & 3 & $\begin{array}{l}1040-1270 \\
\text { (MR12a) }\end{array}$ & $\begin{array}{l}1220-1390 \\
\text { (MR7a) }\end{array}$ & 15 & $10-245$ years & $1232 \mathrm{cal} A D$ \\
\hline & & & \multicolumn{3}{|c|}{ Average of 15 date/10 years IAt datasets } & 14.7/15 (98\%) & 10-240years & $1231 \mathrm{cal} \mathrm{AD}$ \\
\hline & 10 dates & MRR1a & 1 & $\begin{array}{l}\text { 1040-1230 } \\
\text { (MRR6a) }\end{array}$ & $\begin{array}{l}\text { 1220-1300 } \\
\text { (MRR10a) }\end{array}$ & 9 & $15-230$ years & $1218 \mathrm{cal} \mathrm{AD}$ \\
\hline & & & 2 & $\begin{array}{l}1050-1280 \\
\text { (MRR5a) }\end{array}$ & $\begin{array}{l}\text { 1210-1290 } \\
\text { (MRR10a) }\end{array}$ & 10 & $-45-210$ years & $1238 \mathrm{cal}$ AD \\
\hline & & & 3 & $\begin{array}{l}\text { 1040-1270 } \\
\text { (MRR10a) }\end{array}$ & $\begin{array}{l}1220-1300 \\
\text { (MRR7a) }\end{array}$ & 10 & $0-230$ years & $1229 \mathrm{cal}$ AD \\
\hline & & & \multicolumn{3}{|c|}{ Average of 10 date/ 10 years IAt datasets } & $9.7 / 10(97 \%)$ & $-10-225$ years & $1228 \mathrm{cal} \mathrm{AD}$ \\
\hline & 5 dates & MRRR1a & 1 & $\begin{array}{l}\text { 1040-1270 } \\
\text { (MRRR1a) }\end{array}$ & $\begin{array}{l}1210-1290 \\
\text { (MRRR4a) }\end{array}$ & 5 & $-20-225$ years & $1219 \mathrm{cal} \mathrm{AD}$ \\
\hline & & & 2 & $\begin{array}{l}\text { 1040-1270 } \\
\text { (MRRR5a) }\end{array}$ & $\begin{array}{l}\text { 1220-1300 } \\
\text { (MRRR2a) }\end{array}$ & 5 & $5-230$ years & $1219 \mathrm{cal} A D$ \\
\hline & & & 3 & $\begin{array}{l}\text { 1040-1230 } \\
\text { (MRRR5a) }\end{array}$ & $\begin{array}{l}1180-1290 \\
\text { (MRRR2a) }\end{array}$ & 4 & $5-220$ years & $1194 \mathrm{cal}$ AD \\
\hline & & & \multicolumn{3}{|c|}{ Average of 5 date/ 10 years IAt datasets } & $4.7 / 5(94 \%)$ & $-5-225$ years & $1211 \mathrm{cal} \mathrm{AD}$ \\
\hline \multirow[t]{12}{*}{50 Years } & 15 dates & MR1b & 1 & $\begin{array}{l}990-1160 \\
\text { (MR7b) }\end{array}$ & $\begin{array}{l}1210-1290 \\
\text { (MR15b) }\end{array}$ & 10 & $80-275$ years & $1192 \mathrm{cal} \mathrm{AD}$ \\
\hline & & & 2 & $\begin{array}{l}990-1160 \\
\text { (MR10b) }\end{array}$ & $\begin{array}{l}1220-1300 \\
\text { (MR13b) }\end{array}$ & 10 & $85-290$ years & $1183 \mathrm{cal}$ AD \\
\hline & & & 3 & $\begin{array}{l}990-1170 \\
\text { (MR1b) }\end{array}$ & $\begin{array}{l}1220-1380 \\
\text { (MR7b) }\end{array}$ & 8 & $70-280$ years & $1181 \mathrm{cal} \mathrm{AD}$ \\
\hline & & & \multicolumn{3}{|c|}{ Average of 15 date/50 years IAt datasets } & $9.3 / 15(62 \%)$ & $75-285$ years & $1185 \mathrm{cal}$ AD \\
\hline & 10 dates & MRR1b & 1 & $\begin{array}{l}990-1160 \\
\text { (MRR1b) }\end{array}$ & $\begin{array}{l}1170-1290 \\
\text { (MRR9b) }\end{array}$ & 9 & $70-265$ years & $1206 \mathrm{cal}$ AD \\
\hline & & & 2 & $\begin{array}{l}\text { 1030-1210 } \\
\text { (MRR3b) }\end{array}$ & $\begin{array}{l}\text { 1220-1380 } \\
\text { (MRR9b) }\end{array}$ & 8 & $50-260$ years & $1216 \mathrm{cal} \mathrm{AD}$ \\
\hline & & & 3 & $\begin{array}{l}900-1160 \\
\text { (MRR5b) }\end{array}$ & $\begin{array}{l}\text { 1180-1290 } \\
\text { (MRR3b) }\end{array}$ & 6 & $75-285$ years & $1177 \mathrm{cal} \mathrm{AD}$ \\
\hline & & & \multicolumn{3}{|c|}{ Average of 10 date/50 years IAt datasets } & $7.7 / 10(77 \%)$ & $65-270$ years & $1199 \mathrm{cal} \mathrm{AD}$ \\
\hline & 5 dates & MRRR1b & 1 & $\begin{array}{l}\text { 990-1160 } \\
\text { (MRRR2b) }\end{array}$ & $\begin{array}{l}\text { 1220-1300 } \\
\text { (MRRR3b) }\end{array}$ & 3 & $85-290$ years & $1182 \mathrm{cal} \mathrm{AD}$ \\
\hline & & & 2 & $\begin{array}{l}\text { 1040-1230 } \\
\text { (MRRR2b) }\end{array}$ & $\begin{array}{l}\text { 1219-1290 } \\
\text { (MRRR1b) }\end{array}$ & 4 & $10-225$ years & $1194 \mathrm{cal}$ AD \\
\hline & & & 3 & $\begin{array}{l}1020-1200 \\
\text { (MRRR2b) }\end{array}$ & $\begin{array}{l}\text { 1170-1280 } \\
\text { (MRRR3b) }\end{array}$ & 3 & $40-235$ years & $1188 \mathrm{cal} \mathrm{AD}$ \\
\hline & & & \multicolumn{3}{|c|}{ Average of 5 date/50 years IAt datasets } & $3.3 / 5(66 \%)$ & $45-250$ years & $1188 \mathrm{cal} \mathrm{AD}$ \\
\hline
\end{tabular}


Table 11 (continued)

\begin{tabular}{|c|c|c|c|c|c|c|c|c|}
\hline Dataset IAT & Dataset Size & Dataset Code & Run & $\begin{array}{l}\text { Earliest date } \\
\text { (95\% } \\
\text { confidence) }\end{array}$ & $\begin{array}{l}\text { Latest date } \\
\text { (95\% } \\
\text { confidence) }\end{array}$ & $\begin{array}{l}\text { Accurate } \\
\text { dates }(95 \%)\end{array}$ & $\begin{array}{l}\text { Age range } \\
\text { (95\% } \\
\text { probability) }\end{array}$ & $\begin{array}{l}\text { Mean } \\
\text { lifespan }\end{array}$ \\
\hline \multirow[t]{12}{*}{100 Years } & 15 dates & MR1C & 1 & 990-1160 (MR8c) & $\begin{array}{l}\text { 1210-1290 } \\
\text { (MR14C) }\end{array}$ & 9 & $75-270$ years & $1178 \mathrm{cal} \mathrm{AD}$ \\
\hline & & & 2 & $650-800$ (MR4c) & $\begin{array}{l}1220-1300 \\
\text { (MR9c) }\end{array}$ & 6 & $450-625$ years & $1087 \mathrm{cal} \mathrm{AD}$ \\
\hline & & & 3 & $\begin{array}{l}990-1160 \\
\text { (MR10c) }\end{array}$ & $\begin{array}{l}1170-1290 \\
\text { (MR6C) }\end{array}$ & 7 & $65-265$ years & $1143 \mathrm{cal} \mathrm{AD}$ \\
\hline & & & \multicolumn{3}{|c|}{ Average of 15 date/100 years IAt datasets } & 7.3/15 (49\%) & 195-390years & $1136 \mathrm{cal} \mathrm{AD}$ \\
\hline & 10 dates & MRR1C & 1 & $\begin{array}{l}770-1000 \\
\text { (MRR8c) }\end{array}$ & $\begin{array}{l}1170-1280 \\
\text { (MRR9c) }\end{array}$ & 3 & $215-460$ years & $1113 \mathrm{cal} \mathrm{AD}$ \\
\hline & & & 2 & $\begin{array}{l}770-1000 \\
\text { (MRR3C) }\end{array}$ & $\begin{array}{l}\text { 1180-1290 } \\
\text { (MRR10c) }\end{array}$ & 3 & $235-480$ years & $1128 \mathrm{cal} \mathrm{AD}$ \\
\hline & & & 3 & $\begin{array}{l}890-1150 \\
\text { (MRR6c) }\end{array}$ & $\begin{array}{l}1170-1280 \\
\text { (MRR7c) }\end{array}$ & 6 & $110-355$ years & $1168 \mathrm{cal} \mathrm{AD}$ \\
\hline & & & \multicolumn{3}{|c|}{ Average of 10 date/ 100 years IAt datasets } & $4 / 10(40 \%)$ & $185-435$ years & $1136 \mathrm{cal}$ AD \\
\hline & 5 dates & MRRR1C & 1 & $\begin{array}{l}\text { 1030-1210 } \\
\text { (MRRR1C) }\end{array}$ & $\begin{array}{l}1160-1280 \\
\text { (MRRR2C) }\end{array}$ & 3 & $10-225$ years & $1163 \mathrm{cal} \mathrm{AD}$ \\
\hline & & & 2 & $\begin{array}{l}1020-1210 \\
\text { (MRRR3C) }\end{array}$ & $\begin{array}{l}1150-1280 \\
\text { (MRRR5c) }\end{array}$ & 4 & $-10-225$ years & $1180 \mathrm{cal} \mathrm{AD}$ \\
\hline & & & 3 & $\begin{array}{l}\text { 680-950 } \\
\text { (MRRR3c) }\end{array}$ & $\begin{array}{l}\text { 1220-1380 } \\
\text { (MRRR1C) }\end{array}$ & 3 & $335-595$ years & $1109 \mathrm{cal} \mathrm{AD}$ \\
\hline & & & \multicolumn{3}{|c|}{ Average of 5 date/ 100 years IAt datasets } & $3.3 / 5(66 \%)$ & $110-350$ years & $1150 \mathrm{cal} \mathrm{AD}$ \\
\hline \multirow[t]{12}{*}{200 Years } & 15 dates & MR1d & 1 & 700-980 (MR5d) & $\begin{array}{l}1160-1280 \\
\text { (MR15d) }\end{array}$ & 1 & $230-525$ years & $1048 \mathrm{cal} \mathrm{AD}$ \\
\hline & & & 2 & $640-780$ (MR2d) & $\begin{array}{l}1220-1380 \\
\text { (MR13d) }\end{array}$ & 10 & $460-710$ years & $1108 \mathrm{cal} \mathrm{AD}$ \\
\hline & & & 3 & $60-240$ (MR13d) & $\begin{array}{l}1170-1290 \\
\text { (MR4d) }\end{array}$ & 4 & $990-1200$ years & $985 \mathrm{cal} \mathrm{AD}$ \\
\hline & & & \multicolumn{3}{|c|}{ Average of 15 date/200 years IAt datasets } & $5 / 15(33 \%)$ & $560-815$ years & $1047 \mathrm{cal}$ AD \\
\hline & 10 dates & MRR1d & 1 & $\begin{array}{l}\text { 770-1000 } \\
\text { (MRR1d) }\end{array}$ & $\begin{array}{l}1040-1270 \\
\text { (MRR3d) }\end{array}$ & 2 & $85-405$ years & $1098 \mathrm{cal} \mathrm{AD}$ \\
\hline & & & 2 & $\begin{array}{l}670-890 \\
\text { (MRR7d) }\end{array}$ & $\begin{array}{l}1170-1280 \\
\text { (MRR8d) }\end{array}$ & 4 & $320-575$ years & $1097 \mathrm{cal} \mathrm{AD}$ \\
\hline & & & 3 & $\begin{array}{l}550-650 \\
\text { (MRR4d) }\end{array}$ & $\begin{array}{l}1170-1280 \\
\text { (MRR7d) }\end{array}$ & 4 & $550-710$ years & $1035 \mathrm{cal} \mathrm{AD}$ \\
\hline & & & \multicolumn{3}{|c|}{ Average of 10 date/200 years IAt datasets } & $3.3 / 10(33 \%)$ & $315-565$ years & $1076 \mathrm{cal} \mathrm{AD}$ \\
\hline & 5 dates & MRRR1d & 1 & $\begin{array}{l}\text { 440-650 } \\
\text { (MRRR4d) }\end{array}$ & $\begin{array}{l}1150-1280 \\
\text { (MRRR5d) }\end{array}$ & 1 & $525-770$ years & $999 \mathrm{cal} \mathrm{AD}$ \\
\hline & & & 2 & $\begin{array}{l}\text { 670-890 } \\
\text { (MRRR2d) }\end{array}$ & $\begin{array}{l}1210-1290 \\
\text { (MRRR3d) }\end{array}$ & 2 & $360-590$ years & $1085 \mathrm{cal} \mathrm{AD}$ \\
\hline & & & 3 & $\begin{array}{l}\text { 650-780 } \\
\text { (MRRR4d) }\end{array}$ & $\begin{array}{l}\mathbf{9 9 0 - 1 1 6 0} \\
\text { (MRRR5d) }\end{array}$ & 0 & $245-485$ years & $916 \mathrm{cal} \mathrm{AD}$ \\
\hline & & & \multicolumn{3}{|c|}{ Average of 5 date/200 years IAt datasets } & $1 / 5(20 \%)$ & $375-615$ years & $1000 \mathrm{cal} \mathrm{AD}$ \\
\hline
\end{tabular}


Table 11 (continued)

\begin{tabular}{|c|c|c|c|c|c|c|c|c|}
\hline Dataset IAT & Dataset Size & Dataset Code & Run & $\begin{array}{l}\text { Earliest date } \\
\text { ( } 95 \% \\
\text { confidence) }\end{array}$ & $\begin{array}{l}\text { Latest date } \\
\text { ( } 95 \% \\
\text { confidence) }\end{array}$ & $\begin{array}{l}\text { Accurate } \\
\text { dates }(95 \%)\end{array}$ & $\begin{array}{l}\text { Age range } \\
(95 \% \\
\text { probability) }\end{array}$ & $\begin{array}{l}\text { Mean } \\
\text { lifespan }\end{array}$ \\
\hline \multirow[t]{12}{*}{500 years } & 15 dates & MR1e & 1 & $\begin{array}{l}-1020-830 \\
\text { (MR13e) }\end{array}$ & $\begin{array}{l}\text { 1050-1270 } \\
\text { (MR8e) }\end{array}$ & 1 & $1975-2255$ years & $765 \mathrm{cal} \mathrm{AD}$ \\
\hline & & & 2 & $\begin{array}{l}-1410-1220 \\
\text { (MR9e) }\end{array}$ & $\begin{array}{l}\text { 1210-1290 } \\
\text { (MR6e) }\end{array}$ & 1 & $2470-2685$ years & $612 \mathrm{cal} \mathrm{AD}$ \\
\hline & & & 3 & $\begin{array}{l}-1280-1020 \\
\text { (MR1e) }\end{array}$ & $\begin{array}{l}\text { 1160-1280 } \\
\text { (MR9e) }\end{array}$ & 2 & $2240-2525$ years & $719 \mathrm{cal} \mathrm{AD}$ \\
\hline & & & \multicolumn{3}{|c|}{ Average of 15 date/500 years IAt datasets } & $1.3 / 15(8.7 \%)$ & $2225-2490$ years & $699 \mathrm{cal} \mathrm{AD}$ \\
\hline & 10 dates & MRR1e & 1 & $\begin{array}{l}-1120-910 \\
\text { (MRR10e) }\end{array}$ & $\begin{array}{l}1030-1220 \\
\text { (MRR3e) }\end{array}$ & 0 & $1980-2290$ years & $773 \mathrm{cal} \mathrm{AD}$ \\
\hline & & & 2 & $\begin{array}{l}-90-120 \\
\text { (MRR2e) }\end{array}$ & $\begin{array}{l}\text { 1050-1270 } \\
\text { (MRR5e) }\end{array}$ & 2 & 1045-1310 years & $795 \mathrm{cal} \mathrm{AD}$ \\
\hline & & & 3 & $\begin{array}{l}-170-60 \\
\text { (MRR10e) }\end{array}$ & $\begin{array}{l}\text { 1050-1270 } \\
\text { (MRR8e) }\end{array}$ & 2 & $1100-1400$ years & $803 \mathrm{cal} \mathrm{AD}$ \\
\hline & & & \multicolumn{3}{|c|}{ Average of 10 date/500 years IAt datasets } & $1.3 / 10(13 \%)$ & $1375-1670$ years & $790 \mathrm{cal} A D$ \\
\hline & 5 dates & MRRR1e & 1 & $\begin{array}{l}-380-160 \\
\text { (MRRR1e) }\end{array}$ & $\begin{array}{l}\text { 1040-1270 } \\
\text { (MRRR5e) }\end{array}$ & 1 & $1265-1590$ years & $516 \mathrm{cal} \mathrm{AD}$ \\
\hline & & & 2 & $\begin{array}{l}-380-150 \\
\text { (MRRR2e) }\end{array}$ & $\begin{array}{l}\text { 1040-1230 } \\
\text { (MRRR5e) }\end{array}$ & 0 & $1245-1570$ years & $652 \mathrm{cal} \mathrm{AD}$ \\
\hline & & & 3 & $\begin{array}{l}\text { 250-430 } \\
\text { (MRRR4e) }\end{array}$ & $\begin{array}{l}1020-1180 \\
\text { (MRRR3e) }\end{array}$ & 0 & $620-885$ years & $817 \mathrm{cal} \mathrm{AD}$ \\
\hline & & & \multicolumn{3}{|c|}{ Average of 5 date $/ 500$ years IAt datasets } & $0.3 / 5(6 \%)$ & $1040-1350$ years & $662 \mathrm{cal} \mathrm{AD}$ \\
\hline
\end{tabular}

Age range values have been rounded out to 5 years. Inaccurate latest dates are in bold emphasis. Averages are mean values calculated from all three datasets in each group

95\% confidence and four datasets in the study do not contain any accurate simulated dates at all (MRRR1d run 3; MRR1e run 1; MRRR1e runs 2 and 3). Although there is no clear relationship between number of true dates and dataset size for each IAt specified, the earliest latest dates in all five IAt groups are presented by five date datasets, and the latest latest dates have been generated within a 15 date dataset in four of the five IAt groups. Three of the four datasets which do not contain an accurate date are limited to 5 dates (the other is a 10 dater) and three of the four are 500 years IAt.

Age range and age range variation across TS2 generally increases with increased IAt, and with dataset size within each IAt group (Table 11). Narrowly distributed averages of - 5-225 years (MRRR1a) to 10-240 years (MR1a) are presented in datasets specified to 10 years IAt, and much more widely distributed averages of 1040-1350 years (MRRR1e) to 2225-2490 years (MR1e) are presented in datasets specified to 500 years IAt. The average age ranges presented by the 50,100 and 200 years IAt datasets are consistent with this trend, although there is some overlap between individual datasets from all these adjacent groups. The 5 date 100 years IAt group is particularly notable since this contains two datasets with extraordinarily narrow age ranges of $-10-225$ years (MRRR1c run 2) and 10-225 years (MRRR1c run 1), but also extends up to 450-625 years (MR1c run 2). The narrowest age ranges are presented by 5 date datasets in four of the five IAt groups, whilst the widest age ranges are presented by 15 date datasets in three of the five IAt groups. Age range variation is mostly predicated on the earliest simulated date in each dataset, which generally increase in age with increased IAt specification and dataset size; varying from $1053-1273 \mathrm{cal}$ AD in the 10 years IAt dataset (MRR1a, run 2), to 1416-1224 cal BC in the 500 years IAt dataset (MR1e, run 2). Where datasets include very low numbers of dates, and few which include the true event date, then the exponential distribution becomes less visible and more sigmoidal distributions are apparent (Fig. $4 \mathrm{a}-\mathrm{c}$ ).

Mean lifespans are all earlier than the true event date and generally increase in age with the increased IAt specification and age range in TS2 (Table 11). Without rounding out these are all close to expected values. Depending on dataset size, the study presents average mean lifespans of: 1211-1231 cal AD in the group specified with 10 years 
(a) TS2-MR1d-run3

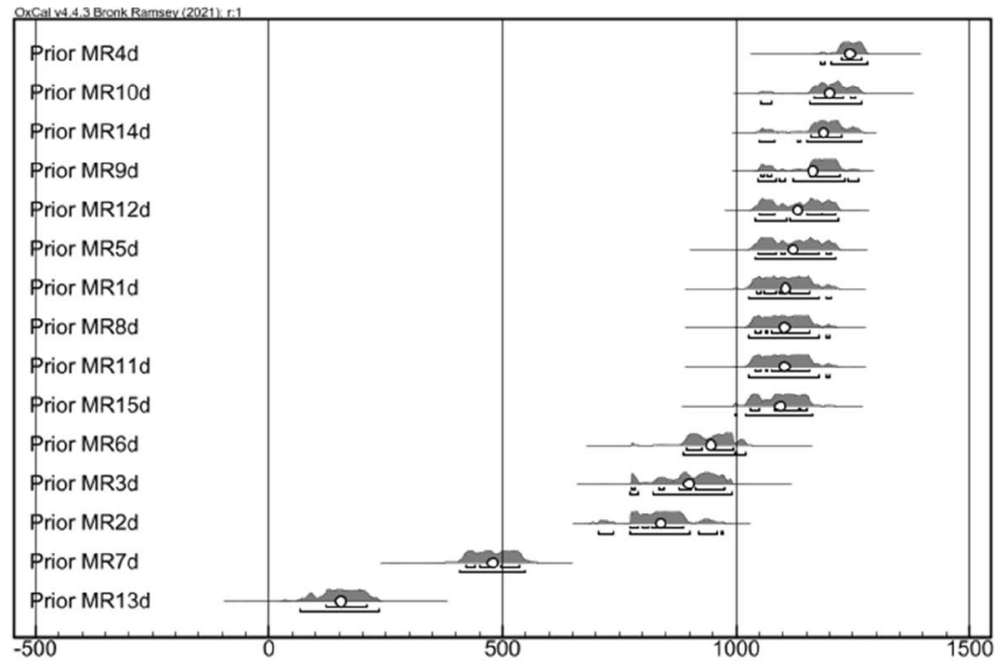

(b) TS2-MRR1d-run3

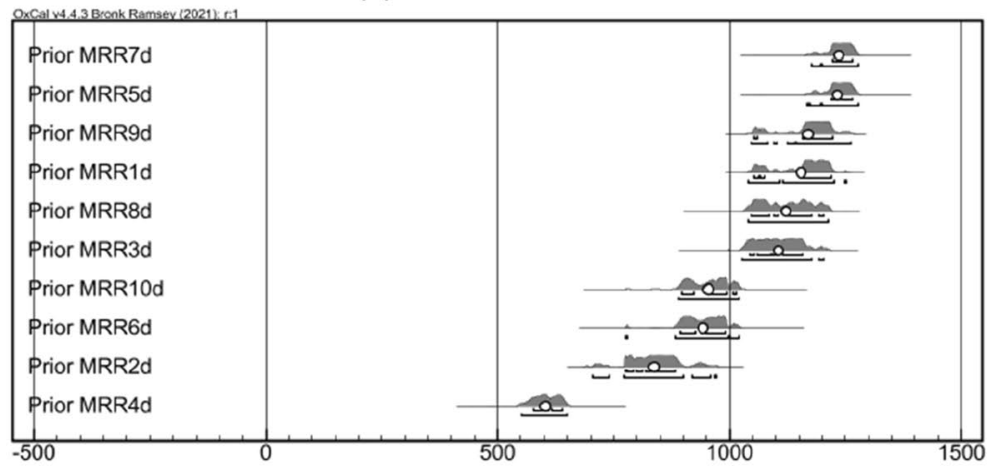

(c) TS2-MRRR1d-run3

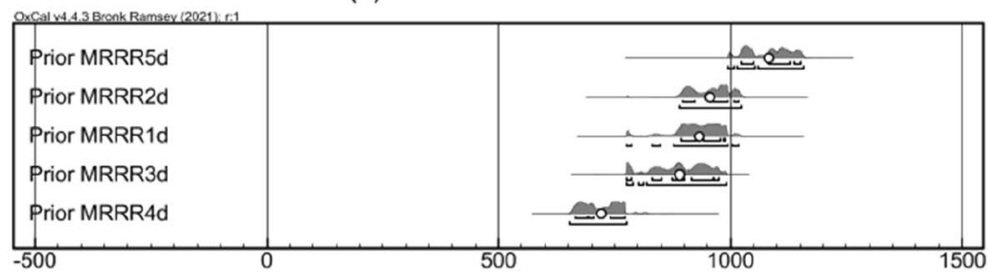

Fig. 4 Selected multiple plots from TS2. With all generated from a true event date of 1250 AD, this includes: subfigure a 15 date 200 years IAt dataset (MR1d run 3); subfigure b 10 date 200 years IAt dataset (MRR1d run 3); and subfigure $\mathbf{c} 5$ date 200 years IAt dataset (MRRR1d run 3). These three plots have been selected to illustrate the decrease in age range and increase in latest date age associated with decreasing dataset size. Dataset MRRR1d run 3 contains no accurate dates at all. Small circles represent the mean average of each distribution

IAt (average 1223 rather than 1240 AD); $1185-1199$ cal AD in the group specified with 50 years IAt (1191 rather than $1200 \mathrm{AD}) ; 1136-1150 \mathrm{cal}$ AD in the group specified with 100 years IAt (1141 rather than 1150 AD); 1000$1076 \mathrm{cal} \mathrm{AD}$ in the group specified with 200 years IAt
(1041 rather than $1050 \mathrm{AD}$ ); and $699-790 \mathrm{cal} \mathrm{AD}$ in the group specified with 500 years IAt (717 rather than 750 AD). Average mean lifespans for each level of specified IAt are all distinct from adjacent groups although, apart from the 500 years IAt, there is some overlap between 
the mean lifespans of individual datasets with those of all other adjacent groups.

All TS2 models have returned Overall Agreement Indices that are above the $60 \%$ threshold (Table 12). $12 \%$ $(16 / 135)$ of these models contain at least one simulated date with a low Agreement Index (Ai) and 4\% (6/135) contain more than one such date. Individual dates with low Agreement Indices are more strongly associated with lower IAt and larger datasets, with no instances in the 500 years IAt or 5 date dataset groups. All six models associated with more than a single low Ai date are associated with two 15 date datasets specified with 10 years IAt (MR1a, runs 2 and 3, all three model specifications).

99\% of all models in TS2 (133/135) have generated accurate End Boundary HPD intervals at 95\% probability, and $84 \%(113 / 135)$ are also accurate at $68 \%$ probability (Table 13). Consistency of End Boundary accuracy varies across the study with dataset IAt, model specification, and dataset size although these relationships are not straightforward. The lowest IAt datasets have produced the most consistently accurate estimates at both $95 \%$ and $68 \%$, but there is no clear overall trend in the relationship between these variables in the rest of the study: all End Boundary HPD intervals generated from datasets specified with 10 years IAt are accurate at both $68 \%$ and $95 \%$ probability; all models associated with the 50 year IAt datasets are also accurate at $95 \%$ probability, but only $7 \mathrm{O} \%$ of these estimates are accurate at $68 \%$ probability; $78 \%$ of models associated with the 100 years and 200 years IAt groups have returned accurate estimates at $68 \%$ probability, but accuracy at $95 \%$ probability is down to $96 \%$ in both cases; while the 500 years IAt group has returned $100 \%$ accuracy at $95 \%$ probability and $93 \%$ accuracy at $68 \%$ probability. The exponential prior/modified Charcoal Outlier Model approach has presented the most consistently accurate End Boundary HPD intervals across the study, with all models $(45 / 45)$ generating accurate estimates at $95 \%$ probability and $87 \%(39 / 45)$ generating accurate estimates at $68 \%$ probability; the uniform prior/default Charcoal Outlier Model approach is also 100\% accurate at $95 \%$ probability, and $80 \%(36 / 45)$ of these models are accurate at $68 \%$ probability; the exponential prior/ no outlier approach has presented accurate estimates in $84 \%$ of models at $68 \%$ probability but is the only approach to present inaccurate End Boundary HPD intervals at 95\% probability. Overall consistency of End Boundary accuracy in TS2 is inversely proportional to dataset size: 100\% (45/45) of all modelled End Boundaries associated with 5 date datasets are accurate at $95 \%$, and $89 \%(40 / 45)$ are also accurate at $68 \%$ probability; 98\% (44/45) of all modelled End Boundaries associated with 10 date datasets are also accurate at $95 \%$, and $84 \%$
$(38 / 45)$ are also accurate at $68 \%$ probability; and $98 \%$ (44/45) of all modelled End Boundaries associated with 15 date datasets are accurate at 95\%, and 78\% (35/45) are also accurate at $68 \%$ probability.

95\% of all models in TS2 (128/135) have generated accurate Last distributions at $95 \%$ probability, and $81 \%$ $(109 / 135)$ are also accurate at $68 \%$ probability (Table 13$)$. Consistency of Last distribution accuracy varies across the study with dataset IAr, model specification, and dataset size although these relationships are not straightforward. The lowest IAt datasets have produced the most consistently accurate estimates at both $95 \%$ and $68 \%$, but there is no clear overall trend in the relationship between these variables in the rest of the study. All Last distributions generated from datasets specified with 10 years.

IAt are accurate at both $68 \%$ and $95 \%$ probability; all models associated with the 50-year and 100-year IAt datasets are also accurate at $95 \%$ probability, but accuracy at $68 \%$ probability decreases to $81 \%$ and $89 \%$ respectively. $81 \%$ of the 200 years IAt groups have returned accurate estimates at $95 \%$ probability, and $75 \%$ of these distributions are also accurate at $68 \% .93 \%$ of the Last distributions generated from the 500-year IAt datasets are accurate at $95 \%$ probability, but only $59 \%$ of this group remains accurate at $68 \%$. The uniform prior/ default Charcoal Outlier Model approach has generated the most consistently accurate Last distributions across TS2 and is the only model specification to generate accurate Last distributions for all (45/45) TS2 datasets at 95\% probability. $87 \%(39 / 45)$ of these estimates are also accurate at $68 \%$ probability. Consistent accuracy is slightly lower for distributions generated using the exponential prior/modified Charcoal Outlier Model approach, with $96 \%$ of Last distributions accurate at $95 \%$ probability and $82 \%(37 / 45)$ at $68 \%$ probability; whilst the exponential prior/no Outlier approach has generated the least consistently accurate Last distributions overall, with $82 \%$ (37/45) of models at 95\% probability and 73\% (33/45) at $68 \%$ probability. In direct contrast with the End Boundary data, overall consistency of Last Distribution accuracy in TS2 is proportional to dataset size: with $98 \%$ $(44 / 45)$ of all generated Last distributions generated from 15 date datasets are accurate at $95 \%$, and $78 \%(35 / 45)$ also accurate at $68 \%$ probability; and $96 \%$ (43/45) of all modelled End Boundaries associated with 10 date datasets are accurate at $95 \%$, and $78 \%(35 / 45)$ are also accurate at $68 \%$ probability; and 91\% (41/45) of all modelled End Boundaries associated with 5 date datasets are accurate at $95 \%$, and $80 \%(36 / 45)$ are also accurate at $68 \%$ probability.

End Boundary precision in TS2 relates strongly to dataset IAt, dataset size and model specification (Table 14). End Boundary precision is inversely proportional to dataset IAt, and the continuous spectra presented by all 


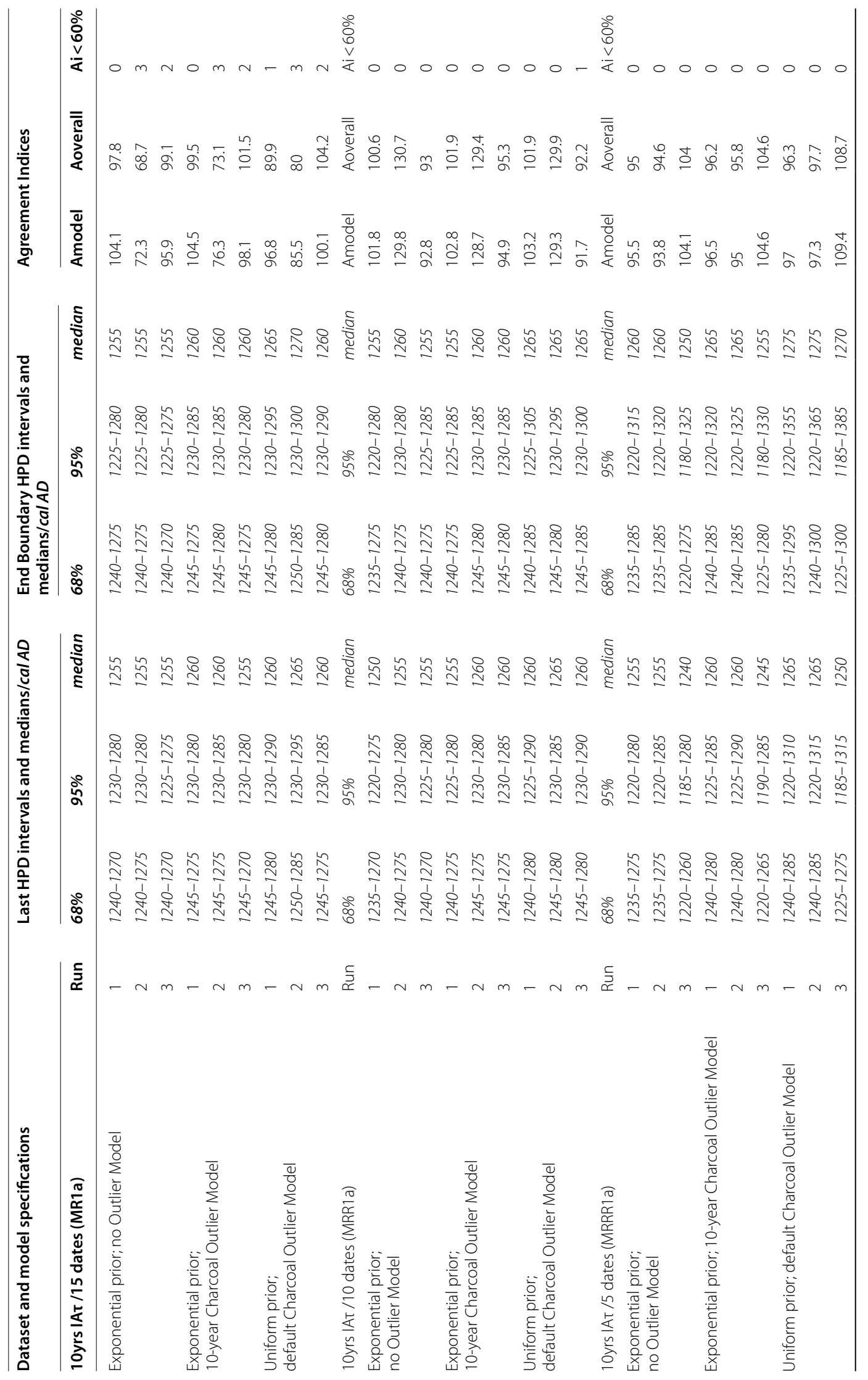




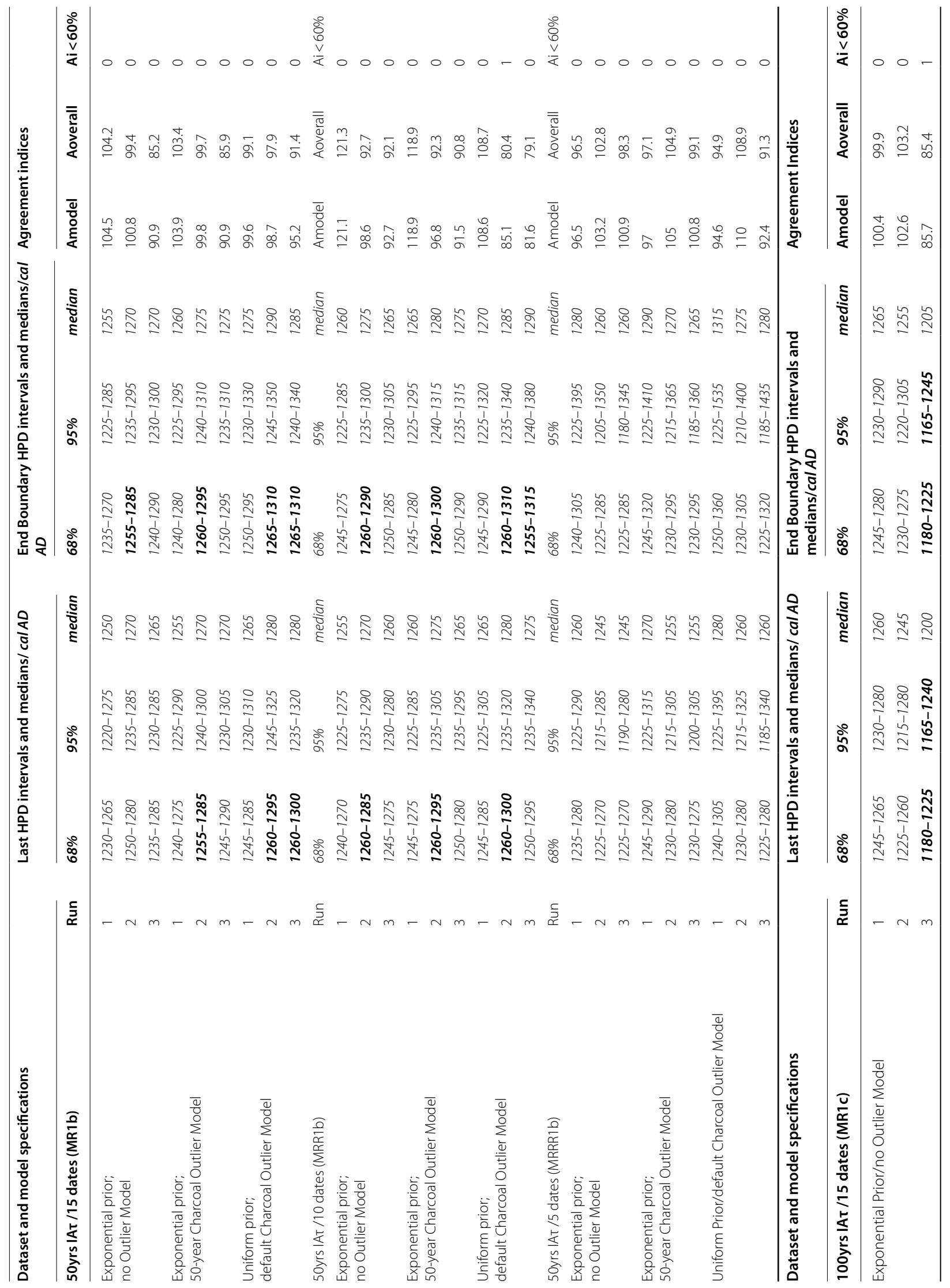




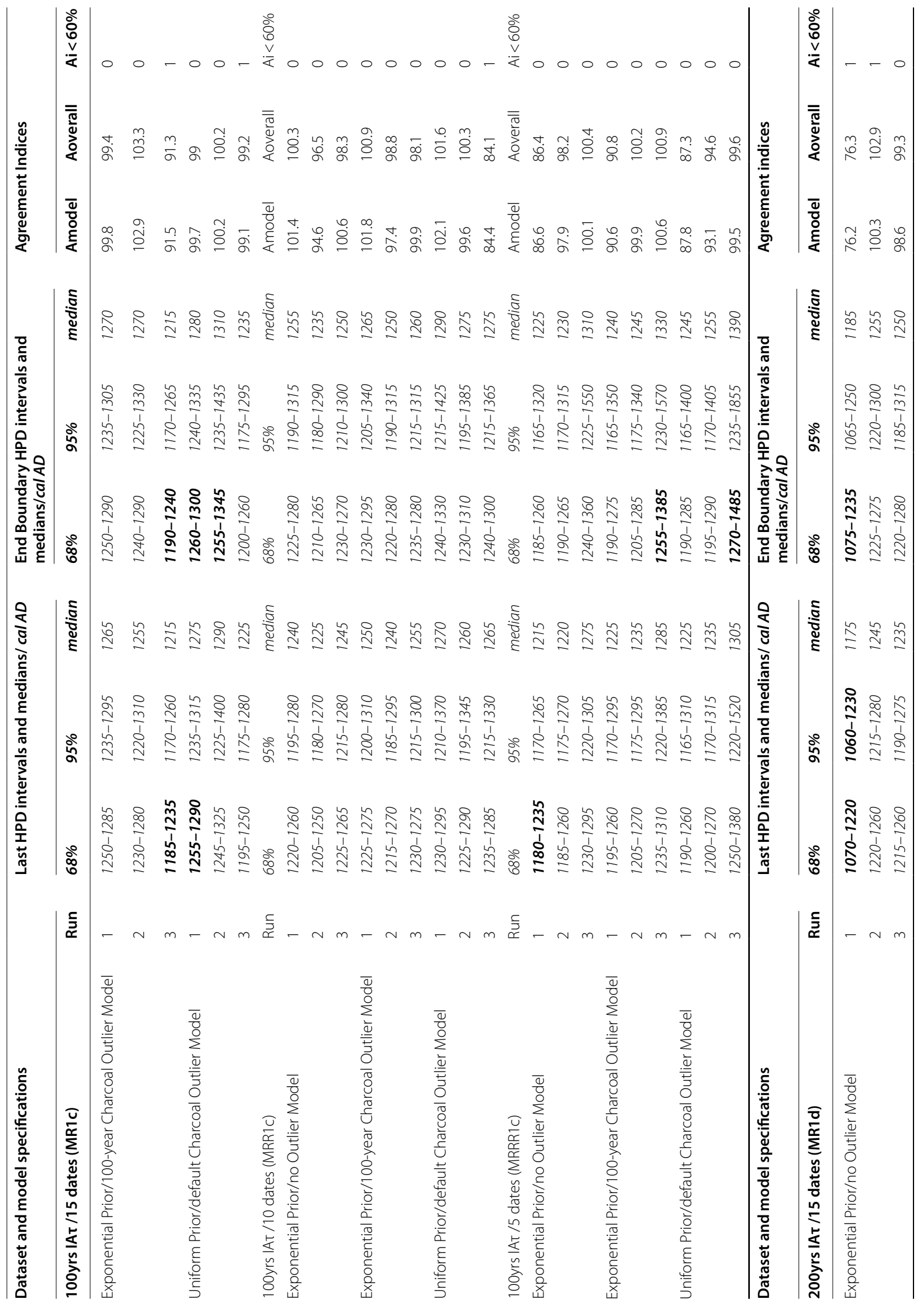




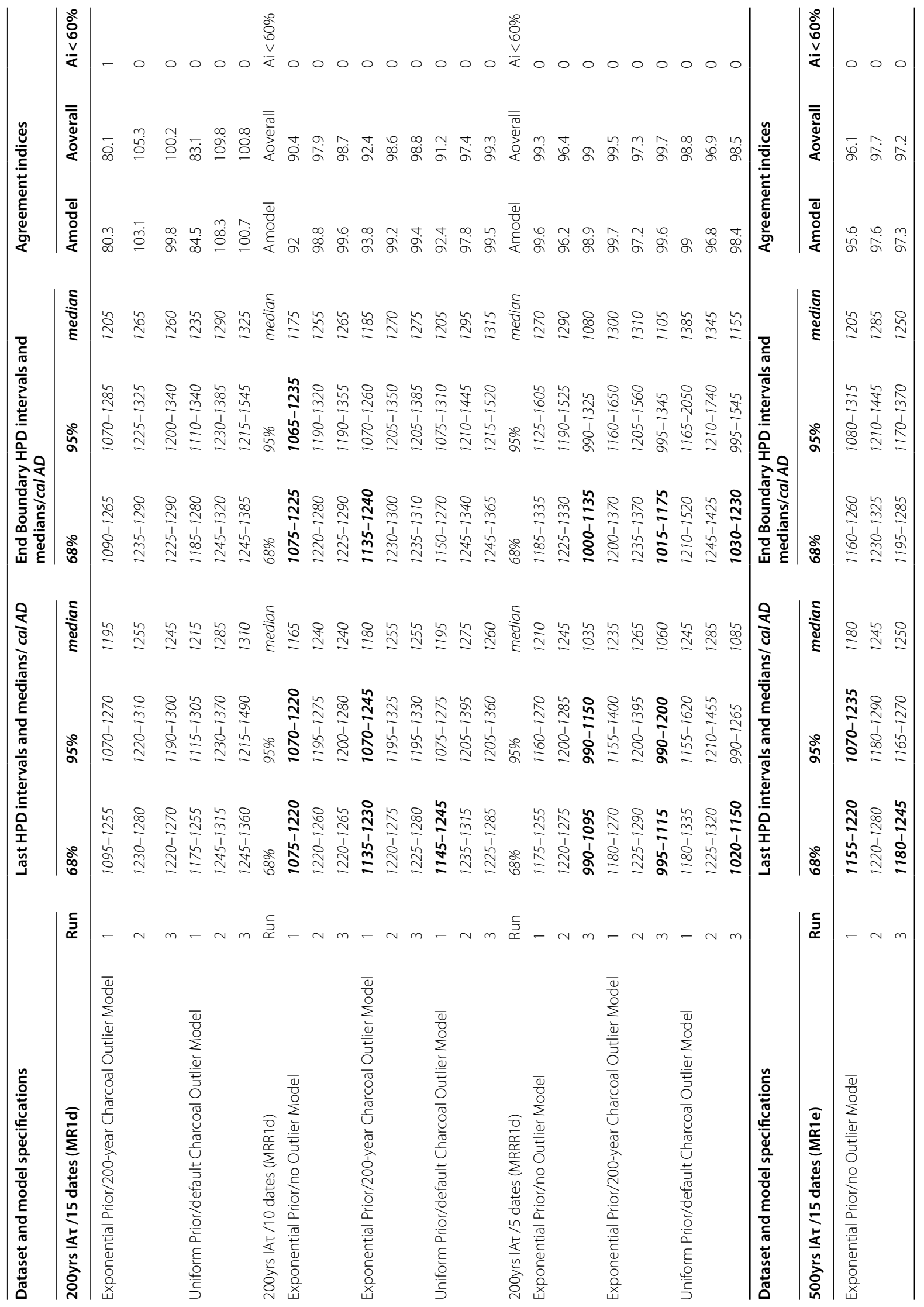




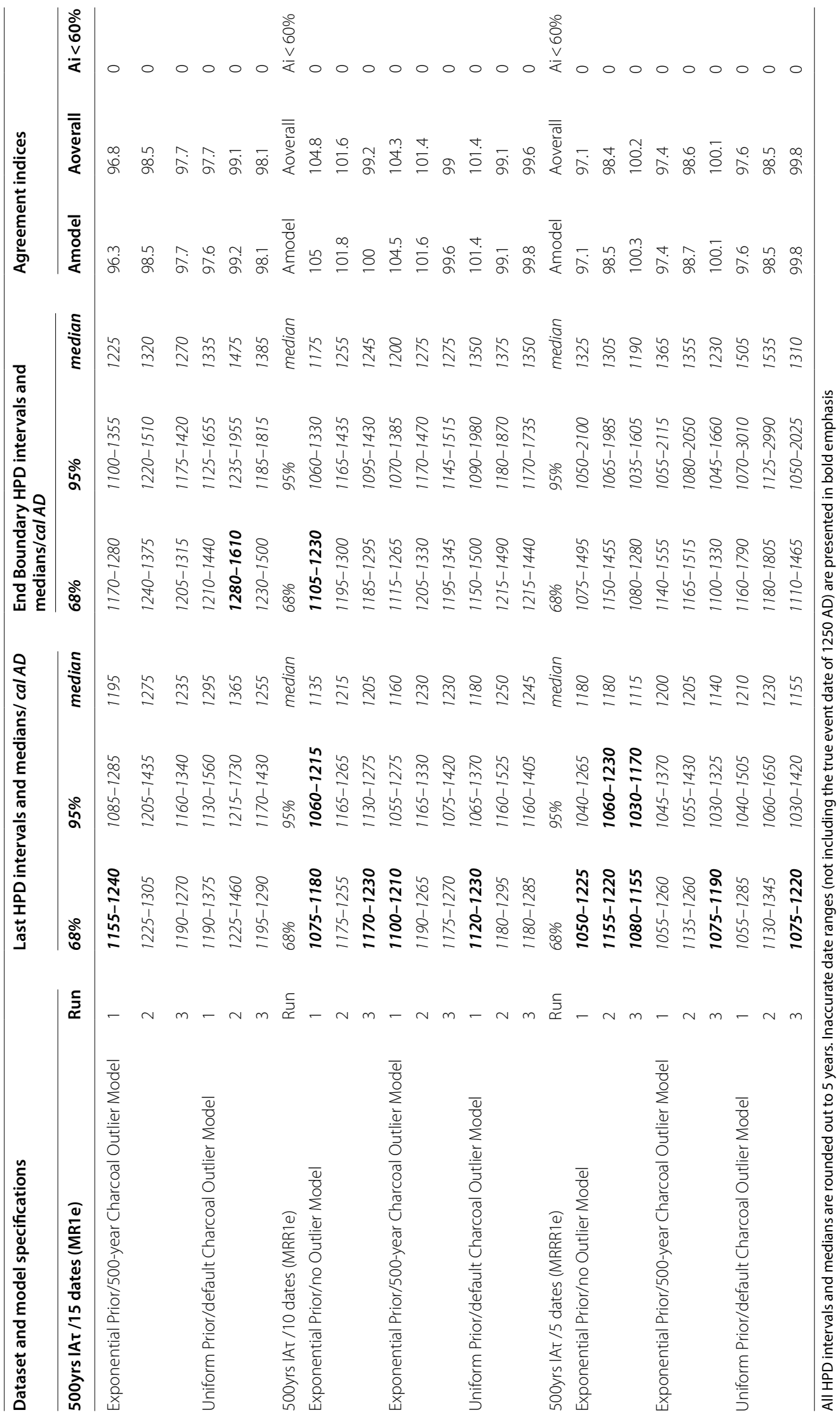




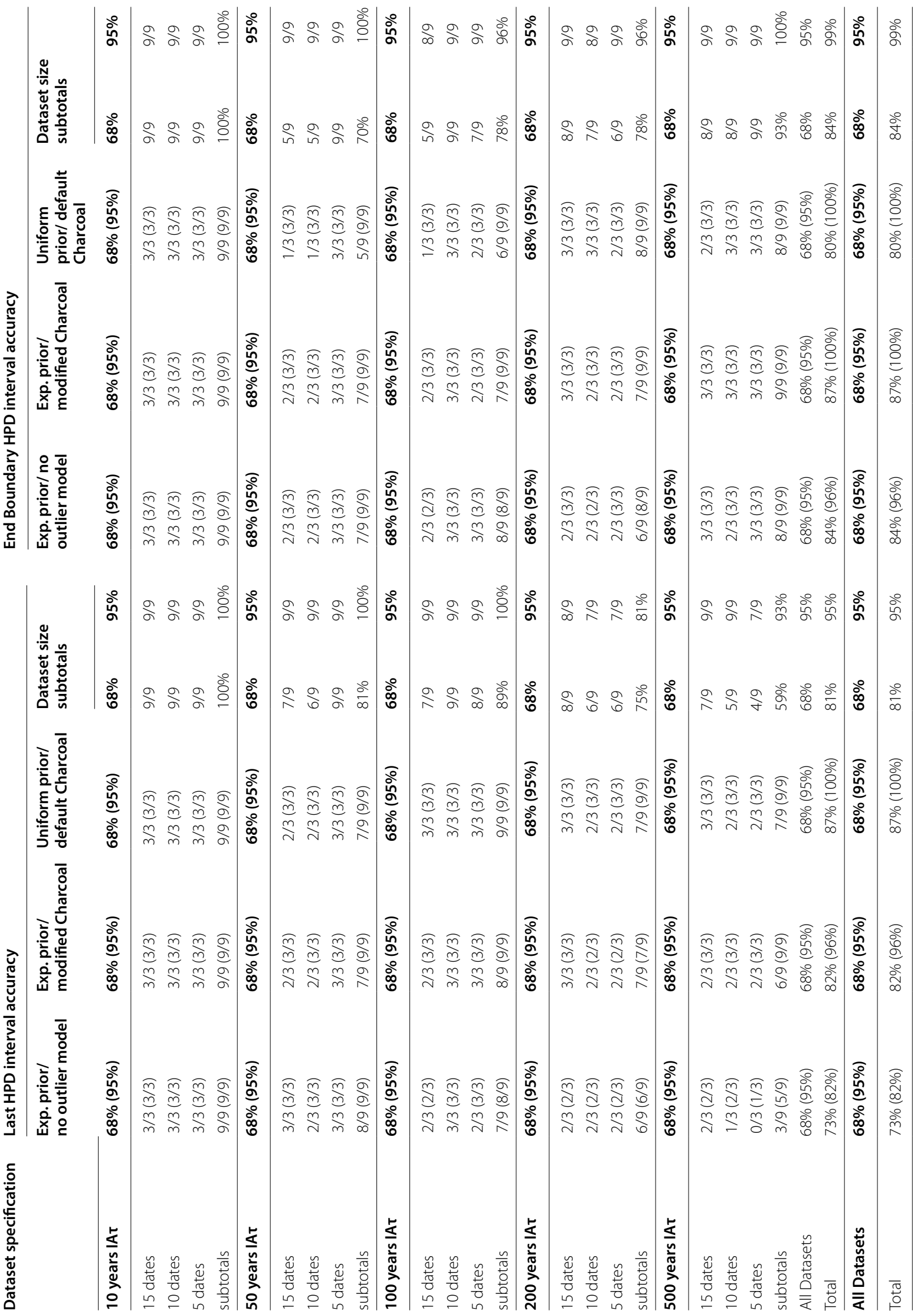


Table 14 Average precision of Last and End Boundary HPD intervals in TS2 (summarised from Table 12)

\begin{tabular}{|c|c|c|c|c|c|c|}
\hline \multirow[t]{2}{*}{$\begin{array}{l}\text { Specified dataset IAt and model } \\
\text { specification }\end{array}$} & \multicolumn{3}{|c|}{ Average precision of Last HPD intervals /years } & \multicolumn{3}{|c|}{$\begin{array}{l}\text { Average precision of End Boundary HPD } \\
\text { intervals/years }\end{array}$} \\
\hline & 15 dates & 10 dates & 5 dates & 15 dates & 10 dates & 5 dates \\
\hline 10 years $\mid A T$ & $68 \%(95 \%)$ & $68 \%(95 \%)$ & $68 \%(95 \%)$ & $68 \%(95 \%)$ & $68 \%(95 \%)$ & $68 \%(95 \%)$ \\
\hline Exp. Prior/no Outlier Model & $32(50)$ & $33(53)$ & $40(73)$ & $33(53)$ & $37(57)$ & $52(113)$ \\
\hline Exp. Prior/10-year Charcoal & $28(52)$ & $32(53)$ & $42(73)$ & $32(53)$ & $35(57)$ & $48(118)$ \\
\hline Uniform Prior/default Charcoal & $33(60)$ & $37(60)$ & $47(105)$ & $35(65)$ & $40(72)$ & $65(160)$ \\
\hline 50 years $\mid A T$ & $68 \%(95 \%)$ & $68 \%(95 \%)$ & $68 \%(95 \%)$ & $68 \%(95 \%)$ & $68 \%(95 \%)$ & $68 \%(95 \%)$ \\
\hline Exp. Prior/no Outlier Model & $32(52)$ & $28(52)$ & $45(75)$ & $38(63)$ & $32(67)$ & $62(160)$ \\
\hline Exp. Prior/50-year Charcoal & $37(67)$ & $32(63)$ & $47(92)$ & $40(72)$ & $38(75)$ & $68(170)$ \\
\hline Uniform Prior/default Charcoal & $38(82)$ & $42(90)$ & $57(145)$ & $45(102)$ & $52(113)$ & $93(250)$ \\
\hline 100 years $\mid A T$ & $68 \%(95 \%)$ & $68 \%(95 \%)$ & $68 \%(95 \%)$ & $68 \%(95 \%)$ & $68 \%(95 \%)$ & $68 \%(95 \%)$ \\
\hline Exp, Prior/no Outlier Model & $33(63)$ & $42(83)$ & $65(92)$ & $42(75)$ & $50(108)$ & $90(208)$ \\
\hline Exp. Prior/100-year Charcoal & $45(80)$ & $50(102)$ & $68(137)$ & $47(90)$ & $57(120)$ & $98(230)$ \\
\hline Uniform Prior/default Charcoal & $57(120)$ & $60(142)$ & $90(197)$ & $63(140)$ & $77(183)$ & $135(363)$ \\
\hline 200 years $\mid A \tau$ & $68 \%(95 \%)$ & $68 \%(95 \%)$ & $68 \%(95 \%)$ & $68 \%(95 \%)$ & $68 \%(95 \%)$ & $68 \%(95 \%)$ \\
\hline Exp. Prior/no Outlier Model & $78(107)$ & 77 (103) & $80(118)$ & $90(132)$ & $92(155)$ & $130(383)$ \\
\hline Exp. Prior/200-year Charcoal & $87(133)$ & $68(147)$ & $92(217)$ & $98(152)$ & $83(172)$ & $155(398)$ \\
\hline Uniform Prior/default Charcoal & $88(202)$ & $80(182)$ & $127(328)$ & $103(238)$ & $112(258)$ & $230(655)$ \\
\hline 500 years $\mid A \tau$ & $68 \%(95 \%)$ & $68 \%(95 \%)$ & $68 \%(95 \%)$ & $68 \%(95 \%)$ & $68 \%(95 \%)$ & $68 \%(95 \%)$ \\
\hline Exp. Prior/no Outlier Model & $63(127)$ & $82(133)$ & $105(178)$ & $95(223)$ & $113(292)$ & $308(847)$ \\
\hline Exp. Prior/500-year Charcoal & $82(203)$ & $93(243)$ & $148(332)$ & $118(263)$ & $142(328)$ & $331(882)$ \\
\hline Uniform Prior/default Charcoal & $172(402)$ & $110(305)$ & $197(482)$ & $277(627)$ & $283(715)$ & $537(1593)$ \\
\hline
\end{tabular}

three dataset sizes for this parameter is notable. In the 15 date models there is a continuous spectrum in End Boundary distributions at $68 \%$ probability from 33 years (10 years IAt) to 277 years (500 years IAt), and at $95 \%$ probability from 53 years ( 10 years IAt) to 627 years (500 years IAt). In the 5 date models there is a continuous spectrum at $68 \%$ probability from 52 years (10 years IAt) to 537 years (500 years IAt), and at $95 \%$ probability from 113 years (10 years IAT) to 1593 years (500 years IAt). End Boundary precision in TS2 is proportional dataset size; and reducing datasets from 15 to 5 dates more than doubles the age range of End Boundary HPD intervals in all models at $95 \%$ probability (whatever the specified dataset IAt), although the contrast between 10 and 5 date datasets accounts for much of this increase. End Boundary precision also varies according to model specification; with the exponential prior/no outlier approach almost invariably presenting the most precise End Boundary HPD intervals at both $68 \%$ and $95 \%$ probabilities (the exception here being the models generated from 10-year IAt datasets) and the uniform prior/default Charcoal Outlier Model approach has always presented the least precise End Boundary date ranges from each dataset. The exponential prior/modified Charcoal Outlier Model approach is generally situated between these two ranges, but closer to the other more precise exponential modelling approach. Each of these effects is cumulative, such that the effects of decreased dataset size and broader model specification on End Boundary precision increases with dataset IAt.

Last distribution precision in TS2 relates strongly to dataset IAt, dataset size and model specification (Table 14). Last distribution precision is inversely proportional to dataset IAt (generally at least doubling between 10-year and 200-year IAt datasets at both $68 \%$ and $95 \%$ probability), and directly proportional to dataset size (Last distributions generated from 5 date datasets are generally 1.5 times broader than 15 date datasets). Again, the difference in Last distribution precision between 10 and 5 dates is considerable and accounts for much of this overall contrast. In general, exponential prior approaches are associated with increased relative precision and Charcoal Outlier Model approaches with decreased precision. All three of these parameters have a cumulative effect on Last distribution precision, such that the 15 date 10 years IAt datasets modelled using the exponential prior/ no outlier approach have generated Last distributions with an average precision of 50 years at $95 \%$ probability and 30 years at $68 \%$ probability; whilst the 5 date 500 years IAt datasets modelled with using the uniform prior/default Charcoal Outlier Model approach have 
generated Last distributions with an average precision of 1593 years at $95 \%$ probability and 537 years at $68 \%$ probability.

Average End Boundary median values in TS2 vary from 1213 cal $A D$ (MRRR1d; exponential prior/no outlier) to 1450 cal $A D$ (MRRR1e; uniform prior/default Charcoal Outlier). End Boundary median values relate directly to model specification; whereby exponential prior distributions are associated with increased median age and the Charcoal Outlier Model approaches are associated with decreased median age (Table 15). End Boundary median values generally decrease in age, and variability increases, with reduced dataset size and increasing IAt.

Average Last distribution median values in TS2 vary from $1158 \mathrm{cal} A D$ (MRRR1e; exponential prior/no outlier) to $1305 \mathrm{cal} A D$ (MR1e; uniform prior/default Charcoal Outlier) (Table 15). These median values relate directly to model specification; whereby exponential prior distributions are generally associated with higher median ages and the Charcoal Outlier Model approaches with lower median ages. Last median values generally increase in age with reduced dataset size, however, and (although more complex) with increasing dataset IAt. This is clearest in the smaller datasets.

\section{Case Study 1 (CS1)—Castle Fincharn main block.}

Documentary evidence suggests some kind of secular building was constructed in the Mid-Argyll settlement of Fincharn between 1240 and 1296 AD, or more certainly 1240-1308 AD [7, 66, 67]. Castle Fincharn has never been excavated, however, and architectural interpretations of the upstanding but fragmentary 2-3 storey structure surviving on the site have varied from the $13^{\text {th }}$ to the sixteenth century. An assemblage of MERLF fragments removed from this building included Quercus sp., Betula sp. and Corylus sp., consistent with regional vegetational histories, and radiocarbon analysis of five widely spaced single entity Corylus sp. samples returned determinations which calibrate to dates ranging from 1050-1270 cal AD (95\% confidence; SUERC-54793) to 1220-1380 cal AD (95\% confidence; SUERC-54796) (Table 16; Fig. 5).

This 5 date MERLF radiocarbon dataset is statistically consistent at $5 \%$ significance level $\left(\mathrm{T}^{\prime}=3.5, \mathrm{~T}^{\prime}(5 \%)=9.5\right.$, $v=4)$; generating a Combine distribution of 1220 1275 cal AD (95\% probability; Fincharn Castle; Additional file 3: Sect. 3.2) and an age range Difference of -40 to 220 years (95\% probability; Fincharn Range; Additional file 3: Sect. 3.1) (Table 16). The Last and End Boundary distributions generated from the dataset range vary between 1230-1285 cal AD (95\% probability) probably

Table 15 Average medians in Last and End Boundary HPD intervals in TS2 (summarised from Table 12)

\begin{tabular}{|c|c|c|c|c|c|c|}
\hline \multirow{2}{*}{$\begin{array}{l}\begin{array}{l}\text { Specified dataset IAT } \\
\text { and model specification }\end{array} \\
10 \text { years IAT }\end{array}$} & \multicolumn{3}{|c|}{ Last HPD interval average median/cal AD } & \multicolumn{3}{|c|}{$\begin{array}{l}\text { End Boundary HPD interval average } \\
\text { median/cal AD }\end{array}$} \\
\hline & 15 dates & 10 dates & 5 dates & 15 dates & 10 dates & 5 dates \\
\hline Exponential prior/no outlier model & 1255 & 1253 & 1250 & 1255 & 1257 & 1257 \\
\hline Exponential prior/10-year Charcoal Outlier Model & 1258 & 1258 & 1255 & 1260 & 1258 & 1252 \\
\hline Uniform prior/default Charcoal Outlier Model & 1262 & 1262 & 1260 & 1265 & 1265 & 1273 \\
\hline 50 years $\mid A \tau$ & 15 dates & 10 dates & 5 dates & 15 dates & 10 dates & 5 dates \\
\hline Exponential prior/no outlier model & 1262 & 1262 & 1250 & 1265 & 1267 & 1267 \\
\hline Exponential prior/50-year Charcoal Outlier Model & 1265 & 1267 & 1260 & 1270 & 1273 & 1275 \\
\hline Uniform prior/default Charcoal Outlier Model & 1275 & 1273 & 1267 & 1283 & 1282 & 1290 \\
\hline 100 years IAT & 15 dates & 10 dates & 5 dates & 15 dates & 10 dates & 5 dates \\
\hline Exponential prior/no outlier model & 1235 & 1237 & 1236 & 1242 & 1247 & 1255 \\
\hline Exponential prior/100-year Charcoal Outlier Model & 1245 & 1248 & 1248 & 1252 & 1265 & 1272 \\
\hline Uniform prior/default Charcoal Outlier Model & 1263 & 1265 & 1255 & 1275 & 1280 & 1297 \\
\hline 200 years $\mid A T$ & 15 dates & 10 dates & 5 dates & 15 dates & 10 dates & 5 dates \\
\hline Exponential prior/no outlier model & 1218 & 1215 & 1163 & 1230 & 1232 & 1213 \\
\hline Exponential prior/200-year Charcoal Outlier Model & 1232 & 1230 & 1187 & 1243 & 1243 & 1238 \\
\hline Uniform prior/default Charcoal Outlier Model & 1270 & 1243 & 1205 & 1283 & 1272 & 1295 \\
\hline 500 years $\mid A T$ & 15 dates & 10 dates & 5 dates & 15 dates & 10 dates & 5 dates \\
\hline Exponential prior/no outlier model & 1225 & 1185 & 1158 & 1247 & 1225 & 1273 \\
\hline Exponential prior/500-year Charcoal Outlier Model & 1235 & 1207 & 1182 & 1272 & 1250 & 1317 \\
\hline Uniform prior/default Charcoal Outlier Model & 1305 & 1225 & 1198 & 1398 & 1358 & 1450 \\
\hline
\end{tabular}


Table16 Radiocarbon results, dataset age ranges and Combine distributions associated with Castle Fincharn main block (CS1)

\begin{tabular}{|c|c|c|c|c|c|c|c|}
\hline \multirow{2}{*}{$\frac{\text { Castle Fincharn }}{\text { Laboratory code }}$} & \multicolumn{5}{|c|}{ Radiocarbon results } & \multicolumn{2}{|c|}{ Modelled distributions } \\
\hline & SUERC-54793 & SUERC-54800 & SUERC-54794 & SUERC-54795 & SUERC-54796 & Datas & Combine range \\
\hline Sample taxon & Corylus & Corylus & Corylus & Corylus & Corylus & range & (cal \\
\hline$\delta^{13} \mathrm{C}(\% 0)$ & -26.6 & -26.9 & -28.7 & -27.5 & 26.4 & & \\
\hline${ }^{14} \mathrm{C}$ age (BP) & $837 \pm 36$ & $808 \pm 36$ & $777 \pm 36$ & $777 \pm 36$ & $744 \pm 36$ & & \\
\hline Calibrated date $68 \%$ probability (cal AD) & $1160-1250$ & $1210-1270$ & $1220-1280$ & $1220-1280$ & $1230-1290$ & 5 to 95 & $1225-1270$ \\
\hline Calibrated date $95 \%$ probability (cal AD) & $1050-1270$ & $1160-1280$ & $1190-1290$ & $1190-1290$ & $1220-1380$ & -40 to 220 & $1220-1275$ \\
\hline
\end{tabular}

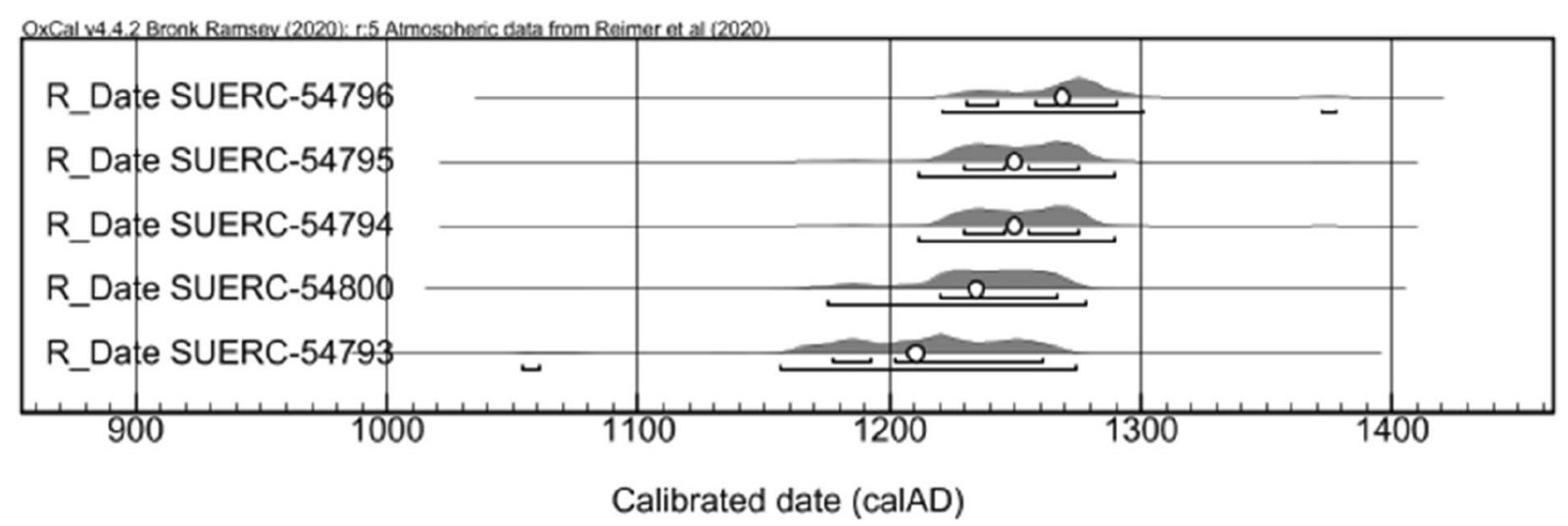

Fig. 5 Unmodelled calibrated probability distributions returned by five MERLF samples from Castle Fincharn main block. Small circles represent the mean average of each distribution

1245-1280 cal AD (68\% probability; Fincharn Lowest IA MERLF 1; Additional file 3: Sect. 3.3), and 1230-1335 cal AD (95\% probability) probably $1245-1295$ cal AD (68\% probability; Castle Fincharn Construction Completed 4; Additional file 3: Sect. 3.6) (Table 17). This includes an exponential prior/modified Charcoal Outlier Model approach specified with a 20 year time-constant consistent with the Corylus-dominated character of the analysed MERLF assemblage (Table 1) and character of the local woodland (Additional file 3: Sect. 3.4).

The upper end of the Combine distribution generated from this Castle Fincharn dataset is not inconconsistent with the historical evidence relating to the site but is relatively early. All Last and End Boundary distributions are also consistent with historical evidence-ranging from: 90\% after TPQ and 100\% before TAQ (Fincharn Lowest IA MERLF 1; Additional file 3: Sect. 3.3) to $96 \%$ after TPQ and $89 \%$ before TAQ (Castle Fincharn Construction Completed 4; Additional file 3: Sect. 3.6). There is little variation in lower limits of all these End Boundary and Last ranges but decreased upper limit ages in End Boundary HPD intervals generated by models which include Charcoal Outlier Models at $95 \%$ probability reduces precision and consistency with historical evidence. All Last ranges are more precise than the latest calibrated date
(SUERC-54796) and, with an estimate-TPQ/TAQ probability sum of $195 \%$, the Last distribution generated by the exponential prior/modified Charcoal Outlier Model approach is the most consistent with currently available historical evidence (Table 17; Fig. 6).

\section{Case Study 2 (CS2)—Aros Castle north-west block}

Documentary evidence suggests some kind of castle building was constructed on the Dun Aros site before 1385 , and current art-historical typologies suggest the bar traceried arcuate windows in the upstanding 2-3 storey north-west block first emerged in Scotland at Elgin Cathedral after 1270 AD [68]. The site has never been excavated and the relationship between the north-west-block and the adjacent enclosure is currently unknown, but architectural comparanda also suggests a late 13th-14th date for this former building is likely. An assemblage of MERLF samples removed from this structure during a wider programme of buildings and materials analysis included fragments of Betula sp., Corylus sp., Fraxinus sp. and Quercus sp. These taxa are consistent with regional vegetational histories, and radiocarbon analysis of five widely spaced single entity Betula and Corylus samples returned determinations which calibrate to dates 


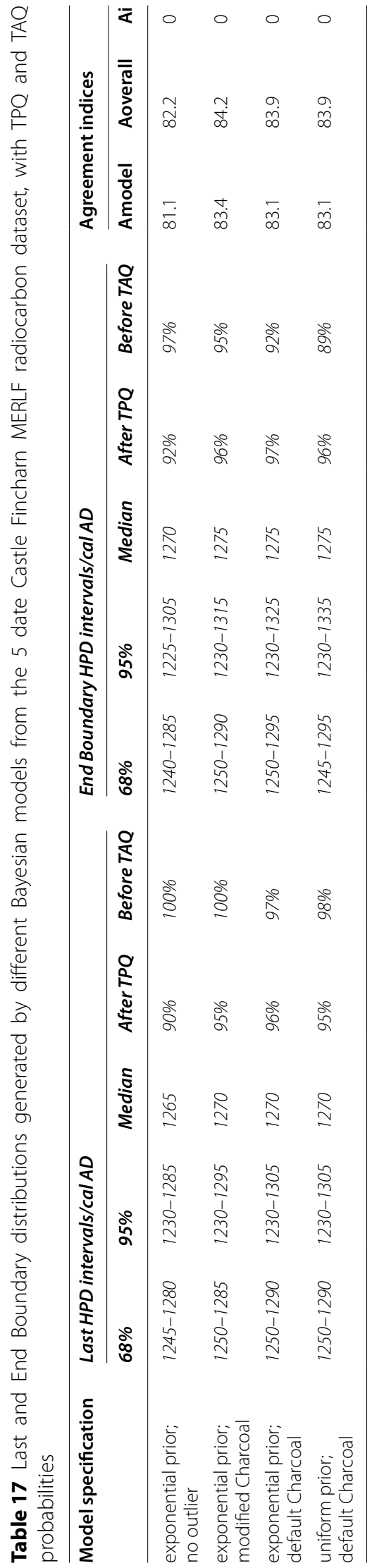




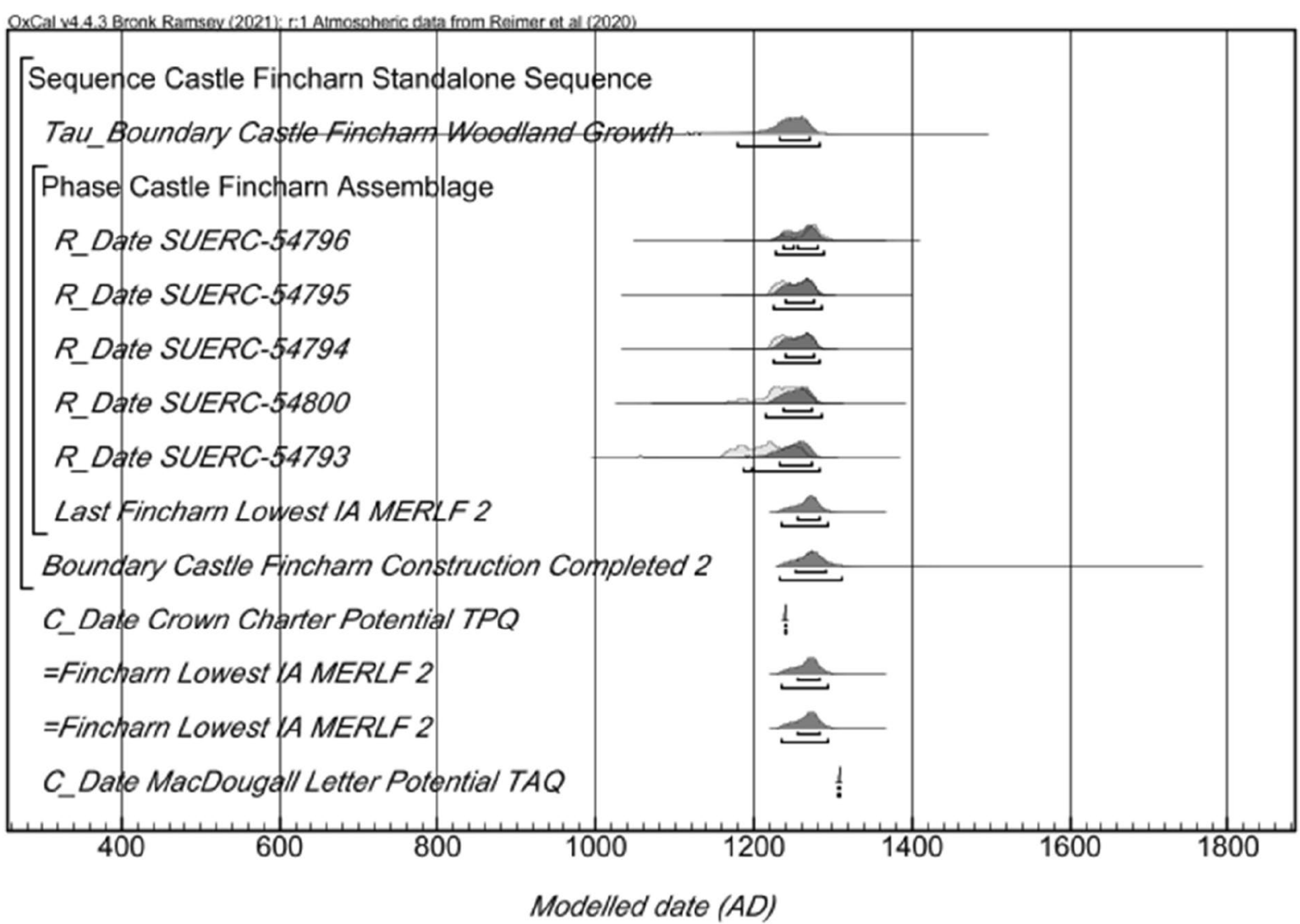

Fig. 6 Probability distributions from a Castle Fincharn main block (CS1) 'standalone' model, generated using the exponential prior/modified Charcoal Outlier approach. All five radiocarbon determinations have been situated within a single exponentially distributed phase and tagged with a 100\% Outlier Probability against a Charcoal Outlier Model specified with a 20 year time-constant

Table 18 Radiocarbon results, dataset age ranges and Combine distributions associated with Aros Castle NW block (CS2)

\begin{tabular}{llllllll}
\hline Aros Castle NW block & \multicolumn{2}{l}{ Radiocarbon results } & & Modelled distributions \\
\hline Laboratory code & SUERC-62567 & SUERC-62563 & SUERC-62564 & SUERC-62565 & SUERC-62566 & $\begin{array}{l}\text { Dataset age } \\
\text { range (years) }\end{array}$ & Combine range \\
(cal AD) & Betula & & \\
Sample taxon & Betula & Betula & Betula & Corylus & Betla & \\
$\delta^{13} \mathrm{C}(\%)$ & -26.6 & -25.8 & -26.9 & -26.3 & -25.4 & \\
${ }^{14} \mathrm{C}$ age (BP) & $804 \pm 34$ & $787 \pm 34$ & $736 \pm 34$ & $657 \pm 34$ & $607 \pm 34$ & \\
Calibrated date 68\% probability (cal AD) & $1220-1270$ & $1220-1280$ & $1260-1300$ & $1289-1390$ & $1300-1400$ & 65 to 150 & $\mathbf{1 2 7 5 - 1 2 8 5}$ \\
Calibrated date 95\% probability (cal AD) & $1170-1280$ & $1180-1290$ & $1220-1380$ & $1270-1400$ & $1290-1410$ & 35 to 190 & $\mathbf{1 2 7 0 - 1 2 9 5}$ \\
\hline
\end{tabular}

Combined ranges are highlighted in bolditalic emphasis as these show poor agreement

ranging between 1170 and 1280 cal $\mathrm{AD}(95 \%$ confidence; SUERC-82567) and 1290-1410 cal AD (95\% confidence; SUERC-62566) (Table 18; Fig. 7).

The current 5 date dataset associated with this building is not statistically consistent at $5 \%$ significance level $\left(T^{\prime}=22.3, T^{\prime}(5 \%)=9.5, v=4\right)$, but generated a Combine distribution of 1270-1295 cal AD (95\% probability; Aros Castle; Additional file 3: Sect. 3.8) and an age range of 35 to 190 years (95\% probability;
Aros Range; Additional file 3: Sect. 3.7) (Table 18). The End Boundary and Last distributions generated from the dataset range between 1290-1395 cal AD (95\% probability) probably $1295-1380$ cal AD $(68 \%$ probability; Aros NW Lowest IA MERLF 1; Additional file 3: Sect. 3.9), and 1290-1595 cal AD (95\% probability) probably $1310-1425$ cal $A D$ (68\% probability; Aros NW Block Construction Completed 4; Additional file 3: Sect. 3.12) (Table 19). This includes an 


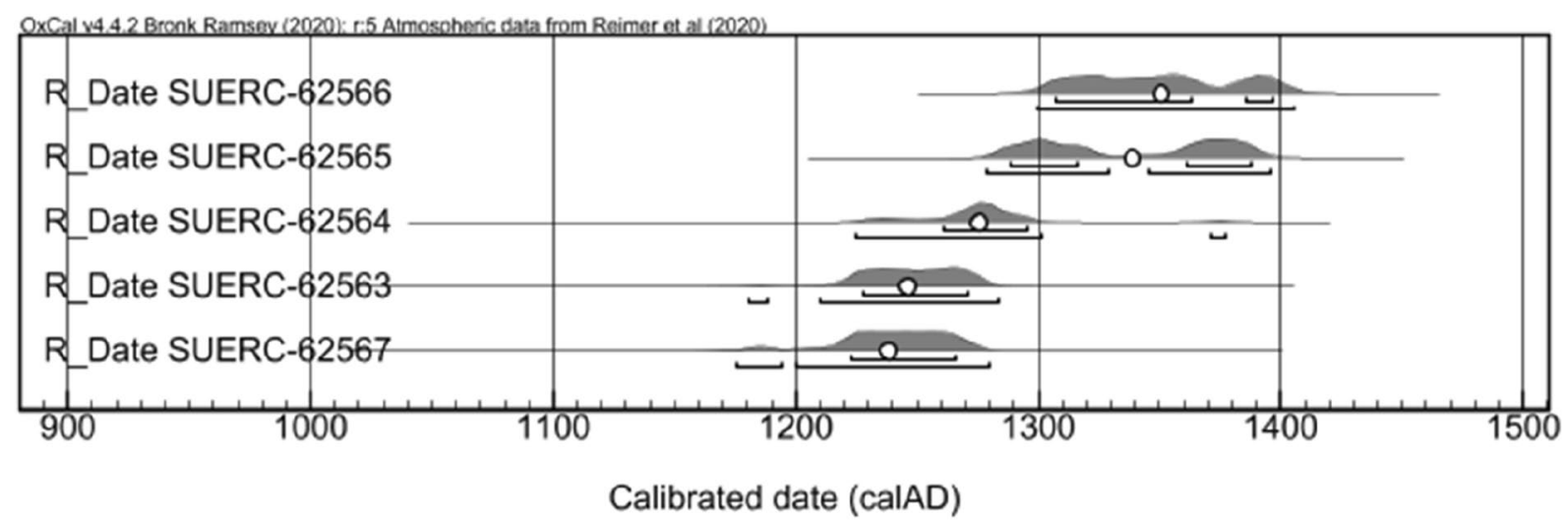

Fig. 7 Unmodelled calibrated probability distributions returned by five MERLF samples from Aros Castle NW block. Small circles represent the mean average of each distribution

exponential prior/modified Charcoal Outlier Model approach specified with a time-constant of 50 years (Additional file 3: Sect. 3.10) consistent with the longest-lived fragments of Betula sp. (here probably $B$. pubescens) (Table 19; Fig. 8).

All estimates generated from this dataset are consistent with the art-historical, architectural, and documentary evidence relating to the building and site, although the Combine distribution is very early. Variation in the lower limit of all other generated distributions is limited to five years at $95 \%$ probability (1295-1300 cal $A D)$, and hence all are $100 \%$ after TPQ. Decreases in the upper limit age (and median) of these Last and End Boundary ranges decreases precision and consistency with historical evidence. With an estimate-TPQ/TAQ probability sum of $193 \%$, the Last distribution generated using the exponential prior/no Outlier approach is most consistent with the available art-historical and historical evidence, presenting a date range very similar to latest dataset date (SUERC-62566; 1290-1410 cal AD at 95\% probability) (Table 19; Fig. 8).

\section{Case Study 3 (CS3)—Castle Roy enclosure and tower}

The Speyside lordship of Abernethy emerges into the surviving documentary record in $1226 \mathrm{AD}$ and the castle enclosure currently surviving on the site of the Castle of Abernethy (now known as Castle Roy) displays an arcuate entrance which is unlikely to have been constructed before 1150 AD [69]. Excavation suggests this substantially upstanding masonry structure is the earliest building on the site, and an extensive assemblage of in-situ MERLF fragments removed from the upstanding castle enclosure included Quercus sp., Betula sp. and Pinus sp. This is broadly consistent with the vegetational history of the region and five widely distributed single entity fragments of Betula and Pinus returned radiocarbon determinations which calibrate to dates ranging between 990 and $1160 \mathrm{cal}$ AD (95\% confidence; SUERC-75745) and 1040-1260 cal AD (95\% confidence; SUERC-75746) (Table 20; Fig. 9).

This 5 date dataset is statistically consistent at 5\% significance $\left(\mathrm{T}^{\prime}=7.2, \mathrm{~T}^{\prime}(5 \%)=9.5, v=4\right)$, but generates a Combine distribution with poor agreement of 1040-1170 cal AD (95\% probability; Castle Roy; Additional file 3: Sect. 3.14) and an age range of -75 to 210 years (95\% probability; Roy Range; Additional file 3: Sect. 3.13) (Table 20). The Last and End Boundary distributions generated from the dataset during this study range between: 1050-1225 cal AD (95\% probability) probably $1155-1220 \mathrm{cal}$ AD (68\% probability; Roy Lowest IA MERLF 1; Additional file 3: Sect. 3.15); and 1055-1415 cal AD (95\% probability) probably 1080-1290 cal AD (68\% probability; Castle Roy Construction Completed 4; Additional file 3: Sect. 3.18) (Table 21), and this includes an exponential prior/modified Charcoal Outlier Model specified with a time-constant of 100 years (Additional file 3: Sect. 3.16), consistent with the shortest-lived fragments of Betula sp. (here probably B. pendula; Table 1).

The extreme upper end of the Combine distribution is consistent with the architectural evidence but is very early. Variation in the lower limits of the generated End Boundary and Last distributions is limited to five years between 1050 and $1055 \mathrm{cal} \mathrm{AD}$ at $95 \%$ probability, and all End Boundary and Last distributions are consistent with the available architectural and historical evidence relating to the building and site. 


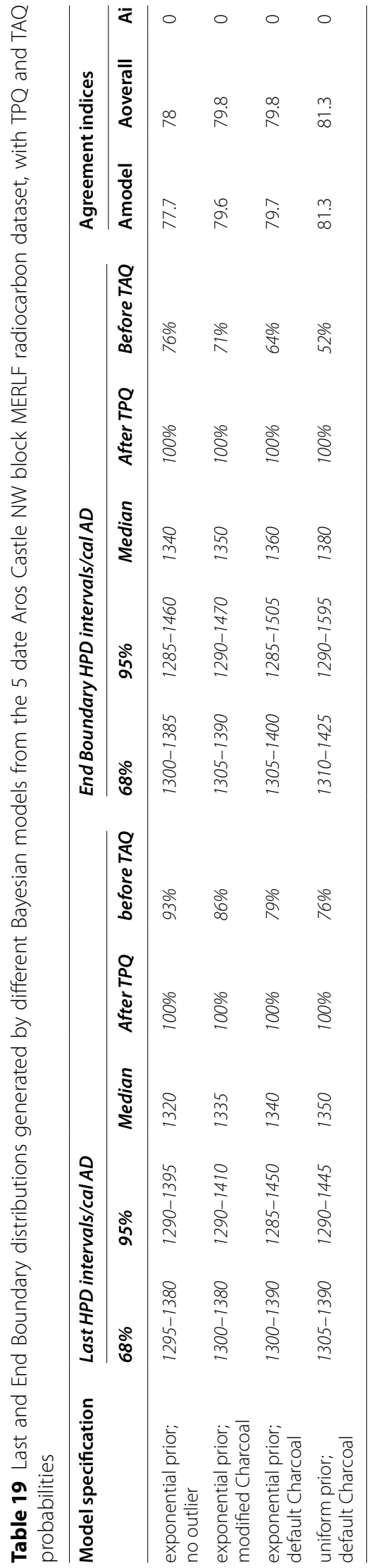




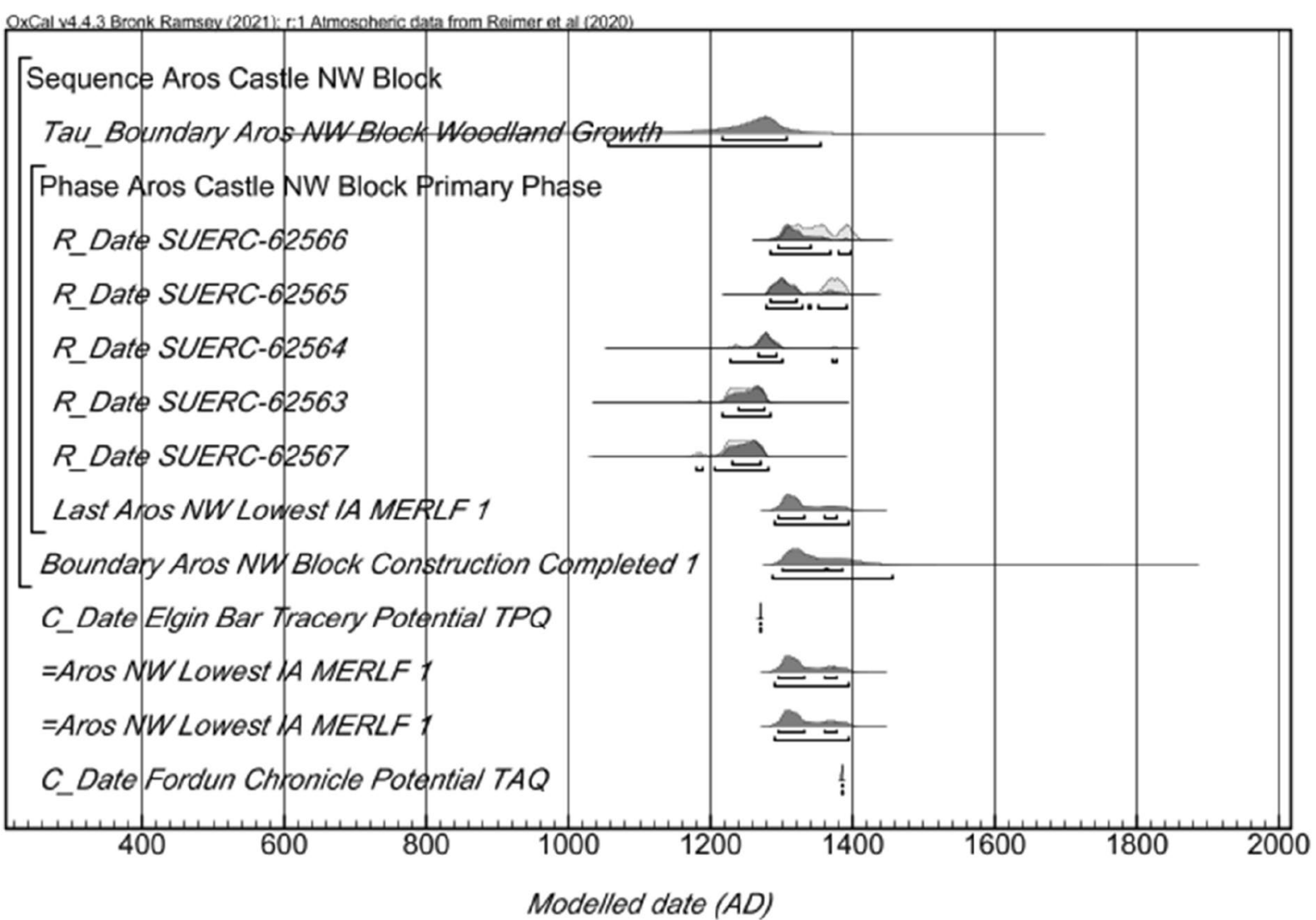

Fig. 8 Probability distributions from an Aros Castle NW block (CS2) 'standalone' model, generated using the exponential prior/no outlier approach. All five radiocarbon determinations have been situated within a single exponentially distributed phase

Table 20 MERLF Radiocarbon results, assemblage age ranges and Combine distributions for Castle Roy (CS3)

\begin{tabular}{|c|c|c|c|c|c|c|c|}
\hline \multirow{3}{*}{$\begin{array}{l}\text { Castle Roy } \\
\text { Laboratory code } \\
\text { Sample taxon }\end{array}$} & \multicolumn{5}{|c|}{ Radiocarbon results } & \multicolumn{2}{|c|}{ Modelled distributions } \\
\hline & SUERC-75745 & SUERC-75743 & SUERC-75744 & SUERC-75742 & SUERC-75746 & Dataset age & Combine range \\
\hline & Pinus & Betula & Pinus & Betula & Betula & range (years) & (cal AD) \\
\hline$\delta^{13} \mathrm{C}(\% \circ)$ & -25.3 & -26.5 & -25.7 & -27.0 & -25.8 & & \\
\hline${ }^{14} \mathrm{C}$ age (BP) & $985 \pm 31$ & $939 \pm 31$ & $905 \pm 31$ & $887 \pm 31$ & $878 \pm 31$ & & \\
\hline $\begin{array}{l}\text { Calibrated date } \\
68 \% \text { probability } \\
\text { (cal AD) }\end{array}$ & $1020-1150$ & $1040-1160$ & $1040-1220$ & $1050-1220$ & $1150-1220$ & 30 to 175 & $1050-1165$ \\
\hline $\begin{array}{l}\text { Calibrated date } \\
95 \% \text { probability } \\
\text { (cal AD) }\end{array}$ & $990-1160$ & $1020-1180$ & $1040-1220$ & $1040-1230$ & $1040-1260$ & -75 to 210 & $1040-1170$ \\
\hline
\end{tabular}

The Combined distribution is highlighted in bolditalic emphasis as this displayed poor agreement

With an estimate-TPQ/TAQ probability sum of $173 \%$, the Last distribution generated using the exponential prior/no outlier approach is most consistent with this evidence, presenting a date range very similar to latest date (SUERC-75746) at $68 \%$ probability, but considerably more precise at $95 \%$ probability (Table 21 ; Fig. 10).
Case Study 4 (CS4) —Lochindorb Castle enclosure

A very narrow 1258-1279 AD constructional date has been widely accepted for initial construction of the upland Moray castle of Lochindorb on the basis that the building was constructed by John Comyn before a 1279 reference to 'Robert of Lochindorb' [7, 


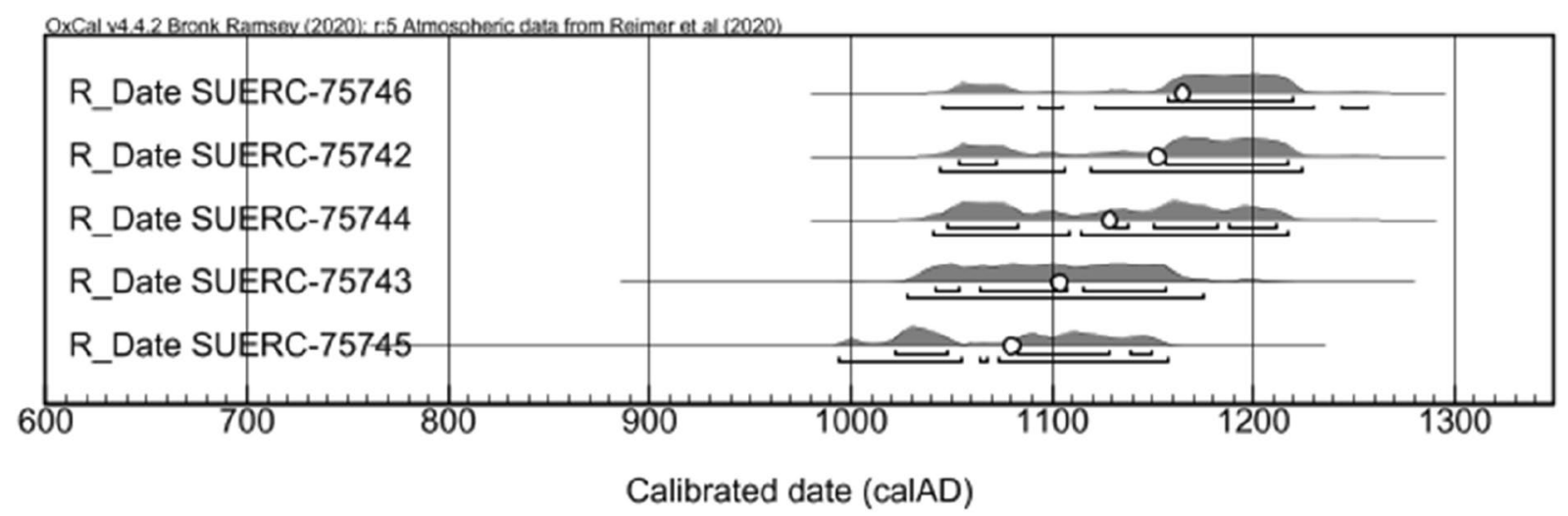

Fig. 9 Unmodelled calibrated probability distributions returned by five MERLF samples from Castle Roy. Small circles represent the mean average of each distribution

70]. A limited assemblage of in-situ MERLF samples removed from the earliest upstanding phase of the enclosure was completely dominated by fragments of Quercus sp., although this genus is not consistent with relict semi-natural woodland populations of Pinus-Betula surviving locally. Five widely spaced single entity Quercus sp. samples with no terminal ring evidence were selected from this phase for radiocarbon analysis, and these returned a wide distribution of calibrated dates ranging between 550 and $380 \mathrm{cal} \mathrm{BC}$ (95\% confidence; SUERC-75752) and 1160-1270 cal AD (95\% confidence; SUERC-75747) (Table 22; Fig. 11).

This 5 date dataset is not statistically consistent at $5 \%$ significance $\left(\mathrm{T}^{\prime}=2014, \mathrm{~T}^{\prime}(5 \%)=9.5, v=4\right)$, generating an age range of 1555 to 1925 years (95\% probability; Lochindorb Range; Additional file 3: Sect. 3.19) and failing to generate a Combine distribution (Additional file 3: Sect. 3.20) (Table 22). The Last and End Boundary distributions generated from this dataset range between 1175-1270 cal AD (95\% probability) probably $1195-1260$ cal AD (68\% probability; Lochindorb Lowest IA MERLF 1; Additional file 3: Sect. 3.21), and 1200-3010 cal AD (95\% probability) probably 1235-1890 cal AD (68\% probability; Construction Lochindorb Castle 4; Additional file 3: Sect. 3.24) (Table 23). This includes an exponential prior/modified Charcoal Outlier Model specified with a timeconstant of 300 years (Additional file 3: Sect. 3.22) consistent with the Quercus sp. dominated character of the MERLF assemblage (Table 1).

All generated Last and End Boundary estimates are consistent with the available historical evidence. With an estimate-TPQ/TAQ probability sum of $114 \%$, the Last distribution generated using the exponential prior/modified Charcoal Outlier Model approach is the most consistent with this other evidence, and this distribution is later and somewhat broader than the latest dataset date (SUERC-75747) (Table 23; Fig. 12).

\section{Case Study 5 (CS5) - Achanduin Castle enclosure and hall}

Surviving charter evidence suggests the upstanding castle at Achanduin on Lismore was constructed between 1240 and $1310 \mathrm{AD}$, whilst a Balliol coin recovered during excavation beneath the castle courtyard has been highlighted to suggest this constructional period may be constrained to a very narrow 12921310 AD period $[49,71]$. A very limited assemblage of in-situ MERLF fragments removed from the upstanding essentially single-phase building was comprised of Quercus sp. and Betula sp., consistent with regional vegetational histories, and radiocarbon analysis of one Quercus and two Betula fragments returned determinations which calibrate to between $1180-1290 \mathrm{cal}$ AD (SUERC-62547) and 1260-1390 cal AD (SUERC62546 ) at $95 \%$ confidence (Table 24; Fig. 13).

This 3 date dataset is statistically consistent at the $5 \%$ significance level $\left(\mathrm{T}^{\prime}=4.0, \mathrm{~T}^{\prime}(5 \%)=6.0, v=2\right)$, generating a Combine distribution of 1265-1295 cal AD (95\% probability; Achanduin Castle; Additional file 3: Sect. 3.26) and an age range of 0 to 155 years (95\% probability; Achanduin Range; Additional file 3: Sect. 3.25) (Table 24). The Last and End Boundary distributions generated from the dataset range between 1270-1385 cal AD (95\% probability) probably 1275-1305 cal AD (68\% probability; Achanduin Lowest IA MERLF 1; Additional file 3: Sect. 3.27), and $1275-1820$ cal $A D(95 \%$ probability) probably 1280-1450 cal AD (68\% probability; Achanduin Castle Construction Completed 4; Additional file 3: Sect. 3.30) (Table 25), and this includes an exponential prior/modified Charcoal Outlier Model specified 


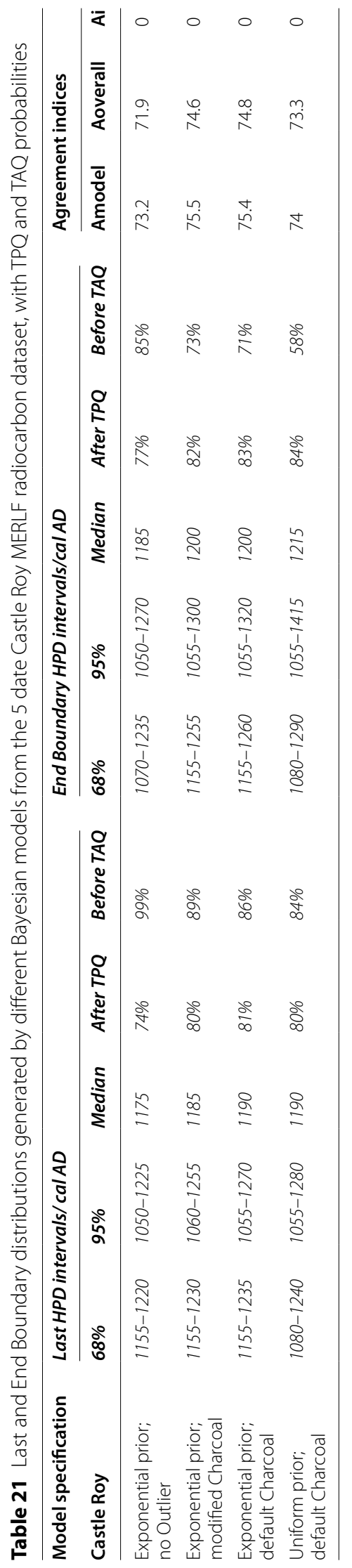




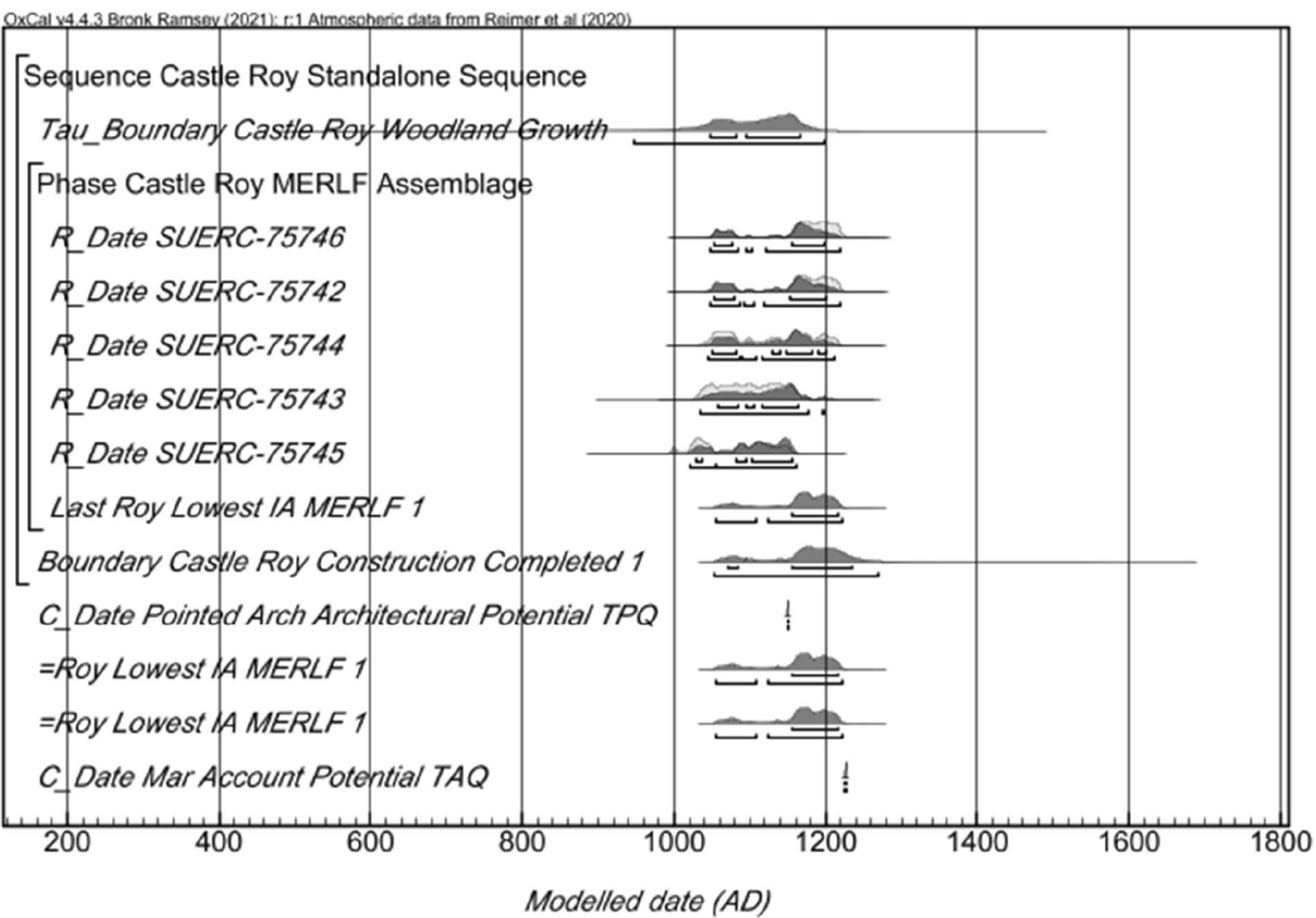

Fig. 10 Probability distributions from an Castle Roy enclosure (CS3) 'standalone' model, generated using the exponential prior/no Outlier approach with all five radiocarbon determinations situated within a single phase

Table 22 MERLF Radiocarbon Determinations, calibrated dates, assemblage age range and Combine distributions for CS4 Lochindorb Castle primary phase enclosure

\begin{tabular}{|c|c|c|c|c|c|c|c|}
\hline Lochindorb Castle & Radiocarbon res & sults & & & & Modelled distributions & \\
\hline Laboratory code & SUERC-75752 & SUERC-75754 & SUERC-75753 & SUERC-75751 & SUERC-75747 & Dataset age range (years) & Combine range \\
\hline Sample taxon & Quercus & Quercus & Quercus & Quercus & Quercus & & \\
\hline$\delta^{13} \mathrm{C}(\% 0)$ & -26.7 & -26.1 & -24.7 & -25.3 & -26.5 & & \\
\hline${ }^{14} \mathrm{C}$ age (BP) & $2368 \pm 31$ & $940 \pm 31$ & $932 \pm 31$ & $862 \pm 31$ & $835 \pm 31$ & & \\
\hline $\begin{array}{l}\text { Calibrated date } 68 \% \text { prob- } \\
\text { ability (cal AD) }\end{array}$ & -480 to -390 & $1040-1160$ & $1040-1160$ & $1160-1220$ & $1170-1260$ & 1600 to 1720 & Failed \\
\hline $\begin{array}{l}\text { Calibrated date 95\% prob- } \\
\text { ability (cal AD) }\end{array}$ & -550 to -380 & $1020-1180$ & $1030-1210$ & $1050-1270$ & $1160-1270$ & 1555 to 1925 & Failed \\
\hline
\end{tabular}

with a time-constant of 50 years (Additional file 3: Sect. 3.28), consistent with the shortest-lived Betula fraction of the MERLF assemblage (Table 1).

The generated Combine distribution is not consistent with the archaeological evidence at $68 \%$ probability. All Last and End Boundary distributions generated are consistent with the available archaeological and historical evidence, with lower limits varying from
1265 to 1275 cal AD and precision and median age decreasing with uniform prior and Charcoal Outlier Model specifications (Table 25). With an estimateTPQ/TAQ probability sum of $141 \%$, the Last distribution generated using the exponential prior/modified Charcoal Outlier Model approach is the most consistent with this other evidence, and this distribution is similar to the latest dataset date (SUERC-62546) at 
OxCal v4.4.2 Bronk Ramsey (2020): r.5 Aimosoheric data from Reimer ot al (2020)

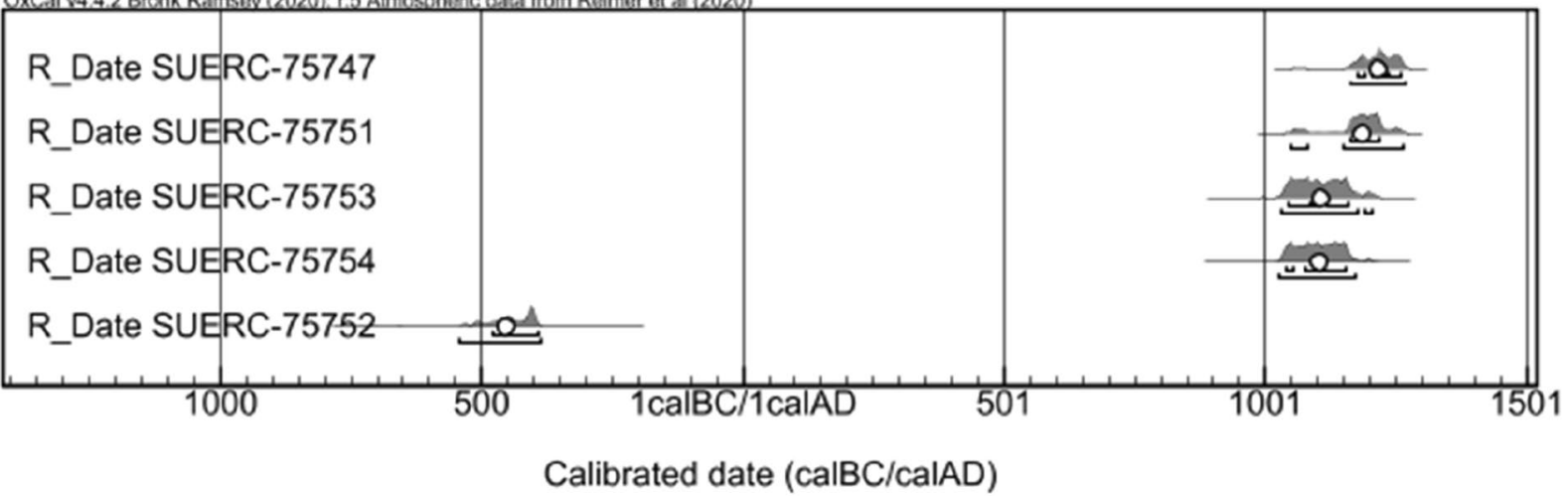

Fig. 11 Unmodelled calibrated probability distributions returned by five MERLF samples from the primary phase enclosure of Lochindorb Castle. Small circles represent the mean average of each distribution

$95 \%$ probability but much more precise at $68 \%$ probability (Table 25; Fig. 14).

\section{Case Study 6 (CS6) - Lismore Cathedral nave}

The earliest surviving contemporary reference to a church building which can be reasonably related to the site of Lismore cathedral dates to $1314 \mathrm{AD}$, although other historical evidence suggests the diocese was formally erected between 1192 and 1214 AD [72-74]. An upstanding medieval church chancel on the site has been ascribed to a range of $13^{\text {th }}$ to fourteenth century dates and highlighted to illustrate the challenges faced by architectural historians in ascribing more precise dates to western Scottish masonry buildings of this period [75]. An assemblage of MERLF samples removed during excavation of the more fragmentary nave and western tower included fragments of Alnus sp., Betula sp., Corylus sp. and Quercus sp. consistent with local vegetational histories, and 3 samples of Corylus and Quercus from the earlier nave returned a range of radiocarbon determinations calibrating to between 1030-1219 cal AD (95\% confidence; SUERC75732) and 1290-1400 (95\% confidence; SUERC75727) (Table 26; Fig. 15). The Corylus MERLF sample (SUERC-75727) within this small assemblage retained some probable terminal ring evidence.

This 3 date dataset is not statistically consistent at $5 \%$ significance $\left(\mathrm{T}^{\prime}=48.9, \mathrm{~T}^{\prime}(5 \%)=6.0, v=2\right)$ but has generated a Combine distribution with poor agreement of 1250-1275 cal AD (95\% probability; Lismore Cathedral Nave; Additional file 3: Sect. 3.32), and an age range of 125 to 345 years (95\% probability; Lismore Range; 4.31) (Table 26). The Last and End Boundary distributions generated from the data range between 1295-1400 cal AD (95\% probability) probably 1300 1395 cal AD (68\% probability; Lismore Nave Lowest
IA MERLF 1; Additional file 3: Sect. 3.33), and 13201905 cal AD (95\% probability) probably 1325-1905 cal $A D$ (68\% probability; Lismore Cathedral Nave Complete 4; Additional file 3: Sect. 3.36). This includes an exponential prior/modified Charcoal Outlier Model specified with a time-constant of 300 years (Additional file 3: Sect. 3.34), consistent with the longest-lived Quercus fraction of the assemblage (Table 1).

The late $13^{\text {th }}$-century Combine date is consistent with available historical evidence. All Last and End Boundary distributions are later than the historical TPQ (100\% probability), but the extent to which these distributions pre-date the documentary TAQ varies between 24\% (Lismore Nave Lowest IA MERLF 1; Additional file 3: Sect. 3.33) and 0\% (Lismore Cathedral Nave Complete 4; Additional file 3: Sect. 3.36). With an estimate-TPQ/TAQ probability sum of $124 \%$, the Last distribution generated using the exponential prior/no outlier approach is the most consistent with this other evidence, and is very similar to the latest dataset date (SUERC-75727) (Table 27; Fig. 16).

\section{Discussion}

\section{The theoretical studies}

Variation in the datasets generated from the same model parameters during these theoretical studies highlights that radiocarbon date simulation is a random probabilistic process, and multiple datasets are therefore required to examine how this variability affects the estimates generated using different modelling approaches. Sixty-five exponentially distributed datasets of between five and twenty simulated dates were randomly generated from a true event of 1250 $\mathrm{AD}$ for the two main theoretical studies considered in this paper-TS1 and TS2. 


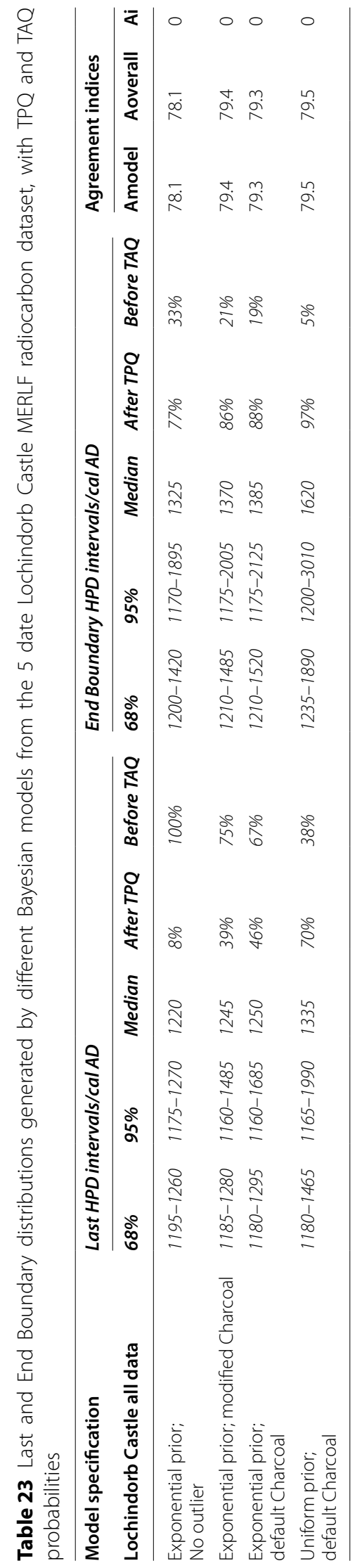




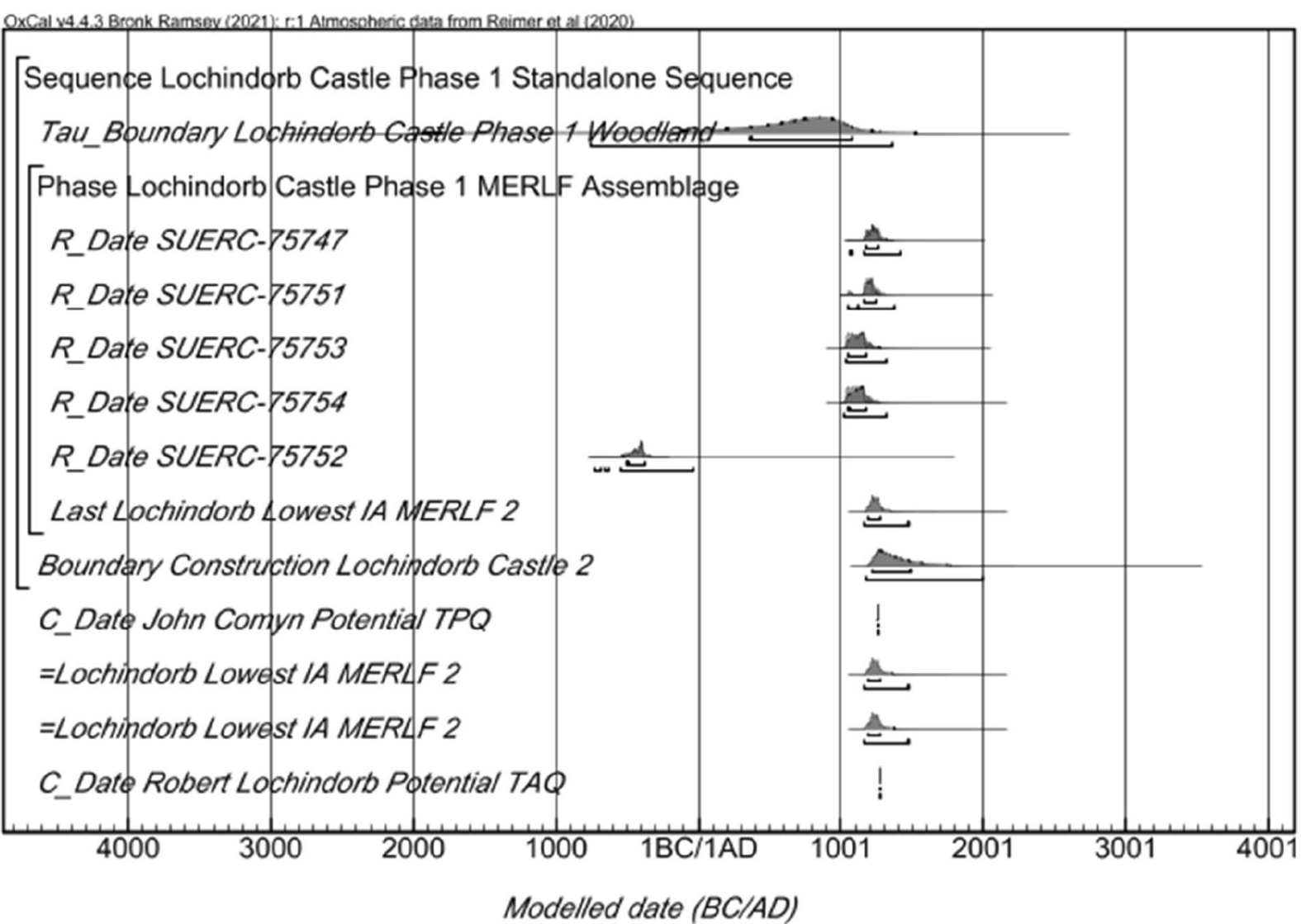

Fig. 12 Probability distributions from a Lochindorb Castle enclosure (CS4) 'standalone' model, generated using the exponential prior/modified Charcoal Outlier Model approach. All five radiocarbon determinations have been situated within a single exponentially distributed phase and tagged with a 100\% Outlier Probability against a Charcoal Outlier Model with a time-constant of 300 years

Table 24 MERLF Radiocarbon results, assemblage age ranges and Combine distributions for Achanduin Castle (CS5)

\begin{tabular}{|c|c|c|c|c|c|}
\hline \multirow{2}{*}{$\begin{array}{l}\text { Achanduin Castle } \\
\text { Laboratory code }\end{array}$} & \multicolumn{3}{|c|}{ Radiocarbon results } & \multicolumn{2}{|c|}{ Modelled distributions } \\
\hline & SUERC-62547 & SUERC-62545 & SUERC-62546 & Dataset age range & Combine \\
\hline Sample taxon & Betula & Betula & Quercus & & range \\
\hline$\delta^{13} \mathrm{C}(\% 0)$ & -25.0 & -26.9 & -25.7 & & \\
\hline${ }^{14} \mathrm{C}$ age (BP) & $785 \pm 34$ & $701 \pm 34$ & $698 \pm 34$ & & \\
\hline Calibrated date $68 \%$ probability (cal AD) & $1220-1280$ & $1270-1380$ & $1270-1380$ & $5-125$ & $1270-1285$ \\
\hline Calibrated date $95 \%$ probability (cal AD) & $1180-1290$ & $1260-1390$ & $1260-1390$ & $0-155$ & $1265-1295$ \\
\hline
\end{tabular}

None of these TS1 or TS2 datasets contain dates which are later than the true event at $95 \%$ probability, and the number of dates in a single dataset which contain the true event at $95 \%$ probability varies from twenty to zero (Tables 2 and 11). Almost all datasets contain at least one date which includes the true event, and this includes all 15 date and 20 date datasets and all datasets with a specified IAt of 100 years or less. Increasing dataset IAt has increased the age of the latest date in both studies and thereby resulted in datasets with a lower fraction of dates which include the true event (Table 28). There is no convincing relationship between fraction of accurate dates and dataset size in these studies, although a drop off is apparent between 10 and 5 date datasets (Table 28) and some small very high IAt datasets are completely dominated by inaccurately early dates (Table 11 ).

Dataset age ranges in these studies are proportional to IAt and size (Table 29). The only 20 date dataset to 


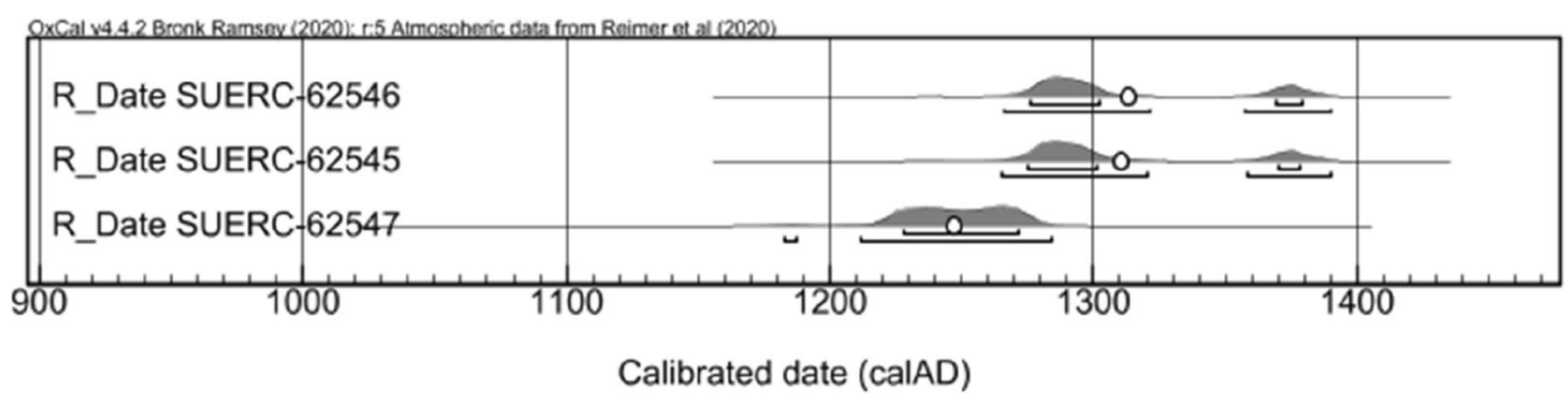

Fig. 13 Unmodelled calibrated probability distributions returned by three MERLF samples from Achanduin Castle. Small circles represent the mean average of each distribution

present an age range with minus values was a 10 years IAt dataset in which all simulated dates contained the true event (TS1 M1a run 1), but three smaller datasets also present minus age range values, including a 5 date 100 years IAt dataset (TS2 MRRR1c run 2) within which four dates include the true event (Tables 2 and $11)$. With some dataset age ranges ranging across thousands of years, it is clear that single determinations from theoretical assemblages subject to IAt do not always directly represent the true event, while the inaccuracy of all Combine distributions generated from 20 date datasets of 50 years IAt or more illustrates that the unweighted averaging of datasets subject to considerable IA does not directly represent this event either. It is salient, however, that the Combine approach can generate accurate distributions from datasets specified with 10 years IAt, at least (Table 3).

251 (98\%) of the 255 models in TS1 and TS2 have generated accurate End Boundary HPD intervals at $95 \%$ probability, and $215(84 \%)$ of these are also accurate at $68 \%$ probability (Table 30 ). Accurate End Boundary estimates have been generated with almost identical consistency in TS1 and TS2, and by the three main models included in both studies (Table 31). End Boundary and Last distributions generated from datasets with very low IA lifespans (10 years IAt) are more consistently accurate in both studies, but no general relationship between accuracy and dataset IA or dataset size was noted elsewhere (Tables 13, 31 and 32). The most consistently accurate End Boundary estimates in both theoretical studies were generated by models specified with both exponential priors and Charcoal Outlier Models (Table 32). This was the only model specification to generate accurate End Boundary HPD intervals at $95 \%$ probability from all datasets in both studies, and in both studies $87-90 \%$ of these estimates were also accurate at $68 \%$ probability. No change in End Boundary accuracy resulted from modifying the Charcoal Outlier Model time-constant (to match that of the specified dataset lifespan) in 20 date models with exponential priors, but an increase in accuracy is evident in the less precise uniform prior approaches (Table 5).

All Last distributions are slightly earlier than the End Boundaries generated from the same datasets in both theoretical studies. These contrasts are more marked in $95 \%$ probability distributions and increase with increasing dataset IAt, decreasing dataset size, and uniform prior and Charcoal Outlier Model specifications. The Last distributions generated in the 5 date to 15 date datasets of TS2 are slightly less consistently accurate than the corresponding End Boundaries (Table 30) and, in further contrast, there is some minor evidence that Last accuracy is proportional to dataset size. Overall, the most consistently accurate Last distributions in TS2 were generated by the uniform prior/default Charcoal Outlier Model approach; and this was the only model specification to generate accurate Last distributions at $95 \%$ probability from all TS2 datasets, with $87 \%$ of these also accurate at $68 \%$ probability (Table 13). That these accuracy percentages are identical to those reported for exponential prior/Charcoal Outlier Model End Boundary distributions is salient and will be returned to below.

Relative precision in End Boundary and Last distributions across both theoretical studies consistently decreases with increasing dataset IAt, decreasing dataset size, and the imposition of a uniform prior distribution or Charcoal Outlier Model, and each of these factors has a cumulative effect. This is illustrated by the average precision of Last and End Boundary distributions generated using the exponential Prior/modified Charcoal Outlier Model approach in TS1 and TS2 where increasing dataset IAt above 100 years or reducing dataset size below 10 dates has considerable impact, even though these datasets 


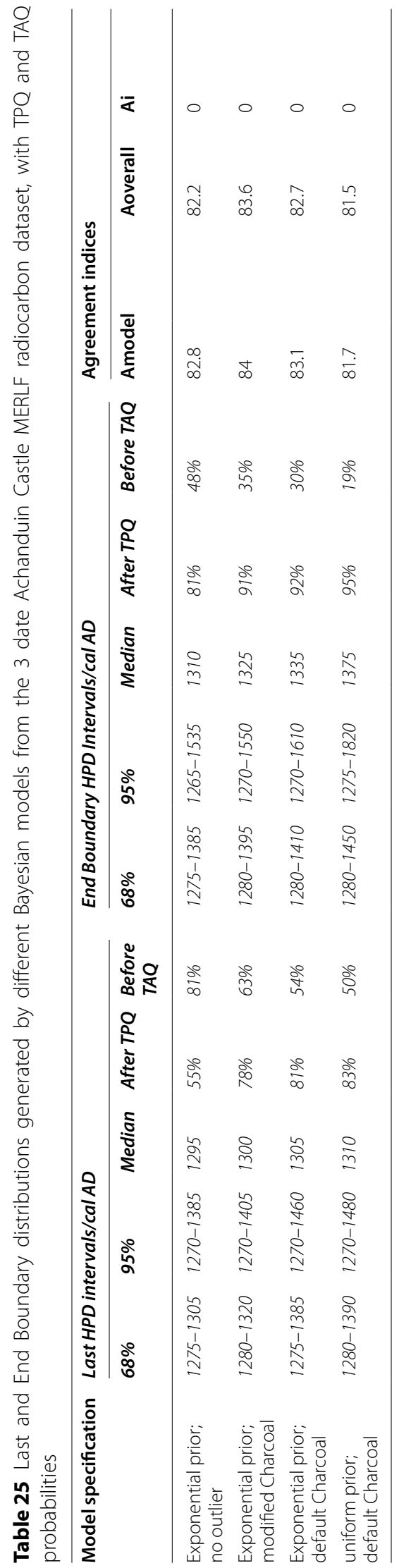




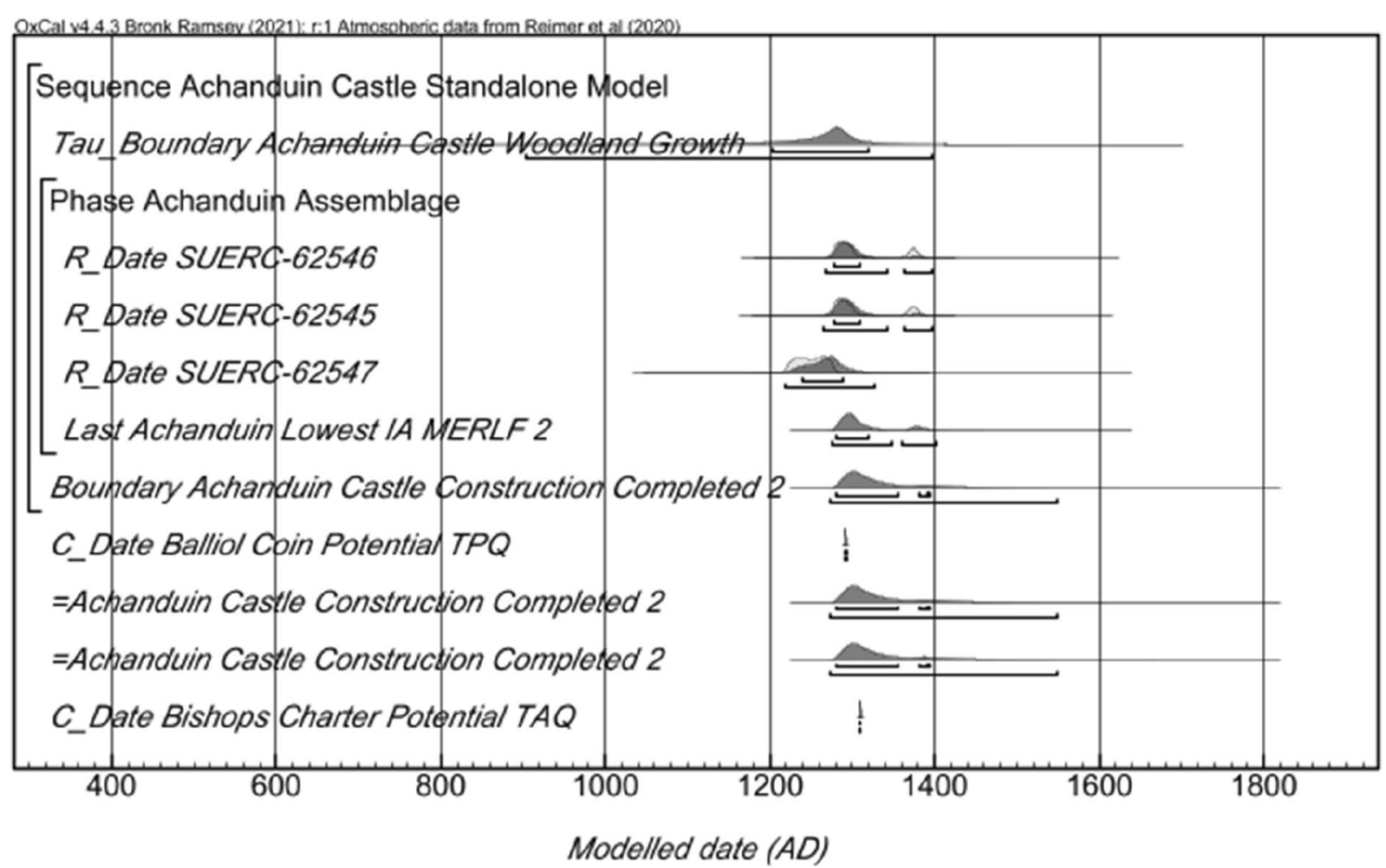

Fig. 14 Probability distributions from an Achanduin Castle (CS5) 'standalone' model, generated using the exponential prior/modified Charcoal Outlier Model approach, All three radiocarbon determinations are situated within a single exponentially distributed phase and tagged with a 100\% Outlier Probability against a Charcoal Outlier Model specified with a time-constant of 50 years

Table 26 Radiocarbon results, dataset age ranges and Combine distributions associated with the MERLF assemblage from Lismore Cathedral nave (CS6)

\begin{tabular}{|c|c|c|c|c|c|}
\hline \multirow{2}{*}{$\begin{array}{l}\text { Lismore Cathedral nave } \\
\text { Laboratory code }\end{array}$} & \multicolumn{3}{|c|}{ Radiocarbon results } & \multicolumn{2}{|c|}{ Modelled distributions } \\
\hline & SUERC-75732 & SUERC-75726 & SUERC-75727 & Dataset age range & Combine range \\
\hline Sample taxon & Quercus & Quercus & Corylus & & (cal AD) \\
\hline$\delta^{13} \mathrm{C}(\%)$ & $-23.9 \%$ & $-25.5 \%$ & $-29.6 \%$ & & \\
\hline${ }^{14} \mathrm{C}$ age (BP) & $921 \pm 30$ & $843 \pm 30$ & $616 \pm 30$ & & \\
\hline Calibrated date $68 \%$ probability (cal AD) & $1040-1170$ & $1170-1260$ & $1300-1400$ & $175-300$ & $1250-1265$ \\
\hline Calibrated date $95 \%$ probability (cal AD) & $1030-1210$ & $1160-1270$ & $1290-1400$ & $125-345$ & $1250-1275$ \\
\hline
\end{tabular}

Combine distributions have been highlighted in bolditalic emphasis as this model presented poor agreement and the dataset failed a chi-square type test

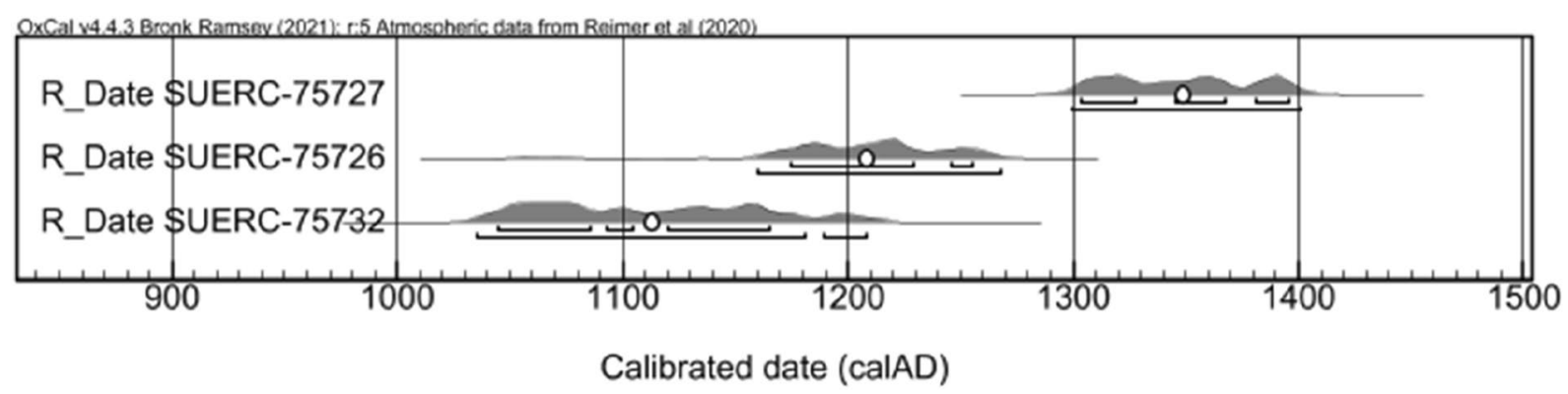

Fig. 15 Unmodelled calibrated probability distributions returned by three MERLF samples from Lismore Cathedral nave. Small circles represent the mean average of each distribution 


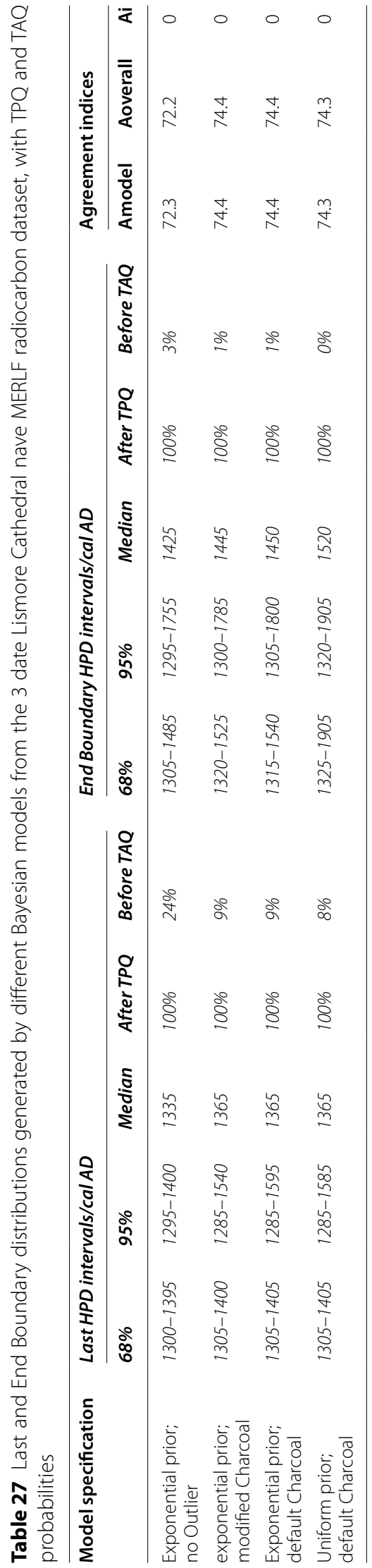




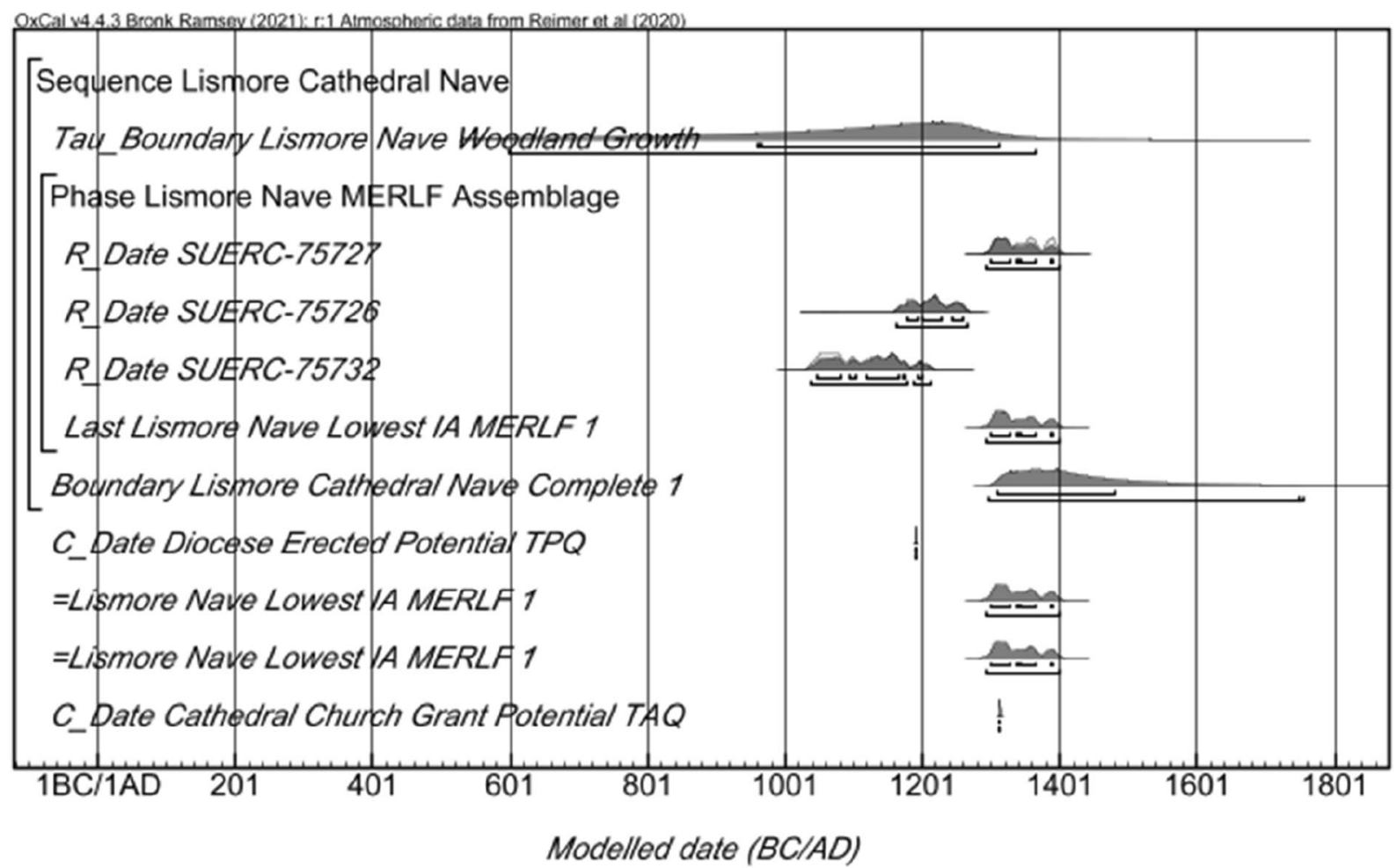

Fig. 16 Probability distributions from a Lismore Cathedral nave'standalone' model, generated using the exponential prior/no outlier approach. All three radiocarbon determinations have been situated within a single exponentially distributed phase

Table 28 Average percentages of accurate dates in TS1 and TS2 datasets (from Tables 2 and 11)

\begin{tabular}{|c|c|c|c|c|c|}
\hline \multirow[t]{2}{*}{ Dataset size } & \multicolumn{5}{|c|}{ Specified dataset IAT } \\
\hline & 10 years & 50 years & 100 years & 200 years & 500 years \\
\hline 20 date & $95 \%(19 / 20)$ & $65 \%(13 / 20)$ & $58 \% 11.5 / 20$ & $25 \%(5 / 20)$ & $12.5 \%(2.5 / 20)$ \\
\hline 15 date & $98 \%(14.7 / 15)$ & $62 \%(9.3 / 15)$ & $49 \% 7.3 / 15$ & $33 \%(5 / 15)$ & $8.7 \%(1.3 / 15)$ \\
\hline 10 date & $97 \%(9.7 / 10)$ & $77 \%(7.7 / 10)$ & $40 \%(4 / 10)$ & $33 \%(3 / 100$ & $13 \%(1.3 / 10)$ \\
\hline 5 date & $94 \%(4.7 / 5)$ & $66 \%(3.3 / 5)$ & $66 \%(3.3 / 5)$ & $20 \%(1 / 5)$ & $6 \%(0.3 / 5)$ \\
\hline
\end{tabular}

Table 29 Average dataset age ranges in TS1 and TS2 (from Tables 2 and 11)

\begin{tabular}{llllll}
\hline Dataset size & \multicolumn{5}{l}{ Specified dataset IAT } \\
\cline { 2 - 6 } & $\mathbf{1 0}$ years & $\mathbf{5 0}$ years & $\mathbf{1 0 0}$ years & $\mathbf{2 0 0}$ years & $\mathbf{5 0 0}$ years \\
\hline 20 date & $5-235$ & $105-325$ & $330-550$ & $465-680$ & $2420-2705$ \\
15 date & $10-240$ & $75-285$ & $195-390$ & $560-815$ & $2225-2490$ \\
10 date & $-10-225$ & $65-270$ & $185-435$ & $315-565$ & $1375-1670$ \\
5 date & $-5-225$ & $45-250$ & $110-350$ & $375-615$ & $1040-1350$ \\
\hline
\end{tabular}

were generated independently (Tables 33 and 34). Last distributions are generally more precise than End Boundary estimates at both $68 \%$ and $95 \%$ probability, and thereby variations in dataset IAt, dataset size, and model specification have a reduced impact. Conversely, given that reducing the Charcoal Outlier Model time-constant appears to have increased precision in broader 20 date TS1 End Boundary distributions subject to uniform priors and high dataset IAT, it is reasonable to expect that time-constant variation would also have a greater effect where dataset size is very reduced and generated estimates broad (see below).

The older latest dates associated with smaller and higher IAt datasets in TS1 and TS2 (Tables 2 and 11) generally result in End Boundaries with older lower limits, and the implications of this relationship for 
Table 30 End Boundary and Last distribution accuracy in all TS1 and TS2 models. (from Tables 5 and 13)

\begin{tabular}{lllll}
\hline Study code & $\begin{array}{l}\text { Accurate end boundaries/ } \\
\mathbf{6 8 \%}\end{array}$ & $\begin{array}{l}\text { Accurate end boundaries/ } \\
\mathbf{9 5 \%}\end{array}$ & $\begin{array}{l}\text { Accurate last distributions/ } \\
\mathbf{6 8 \%}\end{array}$ & $\begin{array}{l}\text { Accurate last } \\
\text { distributions/ } \\
\mathbf{9 5 \%}\end{array}$ \\
\hline TS1 (20 date datasets) & $85 \% ; 102 / 120$ & $98 \% ; 118 / 120$ & \\
TS2 (15-5 date datasets) & $84 \% ; 113 / 135$ & $99 \% ; 133 / 135$ & $81 \% ; 109 / 135$ & $95 \% ; 128 / 135$ \\
Total & $84 \% ; 215 / 255$ & $98 \% ; 251 / 255$ & & \\
\hline
\end{tabular}

Table 31 End Boundary accuracy, model specification and dataset IAt in main models. (from Tables 5 and 13)

\begin{tabular}{|c|c|c|c|c|c|c|}
\hline \multirow[t]{2}{*}{ Specified dataset IAT } & \multirow[t]{2}{*}{ Study code } & \multirow{2}{*}{$\begin{array}{l}\text { Exponential prior; } \\
\text { no outlier } \\
68 \%(95 \%)\end{array}$} & \multirow{2}{*}{$\begin{array}{l}\text { Exp. prior; } \\
\text { modified Charcoal } \\
68 \%(95 \%)\end{array}$} & \multirow{2}{*}{$\begin{array}{l}\text { Uniform prior; } \\
\text { default Charcoal } \\
68 \%(95 \%)\end{array}$} & \multicolumn{2}{|c|}{$\begin{array}{l}\text { All model and } \\
\text { dataset specifications }\end{array}$} \\
\hline & & & & & $68 \%$ & $95 \%$ \\
\hline \multirow[t]{2}{*}{10 years } & TS1 & $4 / 4(4 / 4)$ & $4 / 4(4 / 4)$ & $3 / 4(4 / 4)$ & $38 / 39$ & $39 / 39$ \\
\hline & TS2 & 9/9 (9/9) & $9 / 9(9 / 9)$ & 9/9 (9/9) & & \\
\hline \multirow[t]{2}{*}{50 years } & TS1 & $4 / 4(4 / 4)$ & $4 / 4(4 / 4)$ & $3 / 4(4 / 4)$ & $30 / 39$ & $39 / 39$ \\
\hline & TS2 & $7 / 9(9 / 9)$ & $7 / 9(9 / 9)$ & $5 / 9(9 / 9)$ & & \\
\hline \multirow[t]{2}{*}{100 years } & TS1 & 4/4 (4/4) & 4/4 (4/4) & $2 / 4(4 / 4)$ & $31 / 39$ & $38 / 39$ \\
\hline & TS2 & $8 / 9(8 / 9)$ & $7 / 9(9 / 9)$ & $6 / 9(9 / 9)$ & & \\
\hline \multirow[t]{2}{*}{200 years } & TS1 & $2 / 4(4 / 4)$ & $3 / 4(4 / 4)$ & $4 / 4(4 / 4)$ & $30 / 39$ & $38 / 39$ \\
\hline & TS2 & $6 / 9(8 / 9)$ & $7 / 9(9 / 9)$ & $8 / 9(9 / 9)$ & & \\
\hline \multirow[t]{2}{*}{500 years } & TS1 & $3 / 4(4 / 4)$ & $3 / 4(4 / 4)$ & $2 / 4(3 / 4)$ & $34 / 39$ & $38 / 39$ \\
\hline & TS2 & $8 / 9(9 / 9)$ & 9/9 (9/9) & 9/9 (9/9) & & \\
\hline $\begin{array}{l}\text { All datasets at } \\
68 \%\end{array}$ & & $\begin{array}{l}85 \% \\
55 / 65\end{array}$ & $\begin{array}{l}88 \% \\
57 / 65\end{array}$ & $\begin{array}{l}78 \% \\
51 / 65\end{array}$ & $\begin{array}{l}84 \% \\
163 / 195\end{array}$ & \\
\hline $\begin{array}{l}\text { All datasets at } \\
95 \%\end{array}$ & & $\begin{array}{l}97 \% \\
(63 / 65)\end{array}$ & $\begin{array}{l}100 \% \\
(65 / 65)\end{array}$ & $\begin{array}{l}98 \% \\
(64 / 65)\end{array}$ & & $\begin{array}{l}98 \% \\
192 / 195\end{array}$ \\
\hline $\begin{array}{l}\leq 200-y r s \text { IAt datasets at } \\
68 \%\end{array}$ & & $\begin{array}{l}85 \% \\
44 / 52\end{array}$ & $\begin{array}{l}87 \% \\
45 / 52\end{array}$ & $\begin{array}{l}77 \% \\
40 / 52\end{array}$ & $\begin{array}{l}83 \% \\
129 / 156\end{array}$ & \\
\hline$\leq 200$-yrs IAt datasets at 95\% & & $\begin{array}{l}96 \% \\
(50 / 52)\end{array}$ & $\begin{array}{l}100 \% \\
(52 / 52)\end{array}$ & $\begin{array}{l}100 \% \\
(52 / 52)\end{array}$ & & $\begin{array}{l}99 \% \\
154 / 156\end{array}$ \\
\hline
\end{tabular}

Table 32 Accuracy of different model specs and dataset sizes in TS1 and TS2 (from Tables 5 and 13)

\begin{tabular}{|c|c|c|c|c|c|c|c|}
\hline \multirow[t]{2}{*}{ Model specification } & \multicolumn{3}{|l|}{ Last } & \multicolumn{4}{|c|}{ End Boundary } \\
\hline & 15 date & 10 date & 5 date & 20 date & 15 date & 10 date & 5 date \\
\hline exponential prior/no outlier & $\begin{array}{l}12 / 15 \\
(12 / 15)\end{array}$ & $\begin{array}{l}11 / 15 \\
(13 / 15)\end{array}$ & $\begin{array}{l}10 / 15 \\
(12 / 15)\end{array}$ & $\begin{array}{l}17 / 20 \\
(20 / 20)\end{array}$ & $\begin{array}{l}12 / 15 \\
(14 / 15)\end{array}$ & $\begin{array}{l}12 / 15 \\
(14 / 15)\end{array}$ & $\begin{array}{l}14 / 15 \\
(15 / 15)\end{array}$ \\
\hline exponential prior/ Charcoal Outlier & $\begin{array}{l}12 / 15 \\
(15 / 15)\end{array}$ & $\begin{array}{l}12 / 15 \\
(14 / 15)\end{array}$ & $\begin{array}{l}13 / 15 \\
(14 / 15)\end{array}$ & $\begin{array}{l}18 / 20 \\
(20 / 20)\end{array}$ & $\begin{array}{l}13 / 15 \\
(15 / 15)\end{array}$ & $\begin{array}{l}13 / 15 \\
(15 / 15)\end{array}$ & $\begin{array}{l}13 / 15 \\
(15 / 15)\end{array}$ \\
\hline uniform prior/ Charcoal Outlier & $\begin{array}{l}14 / 15 \\
(15 / 15)\end{array}$ & $\begin{array}{l}12 / 15 \\
(15 / 15)\end{array}$ & $\begin{array}{l}13 / 15 \\
(15 / 15)\end{array}$ & $\begin{array}{l}14 / 20 \\
(19 / 20)\end{array}$ & $\begin{array}{l}10 / 15 \\
(15 / 15)\end{array}$ & $\begin{array}{l}13 / 15 \\
(15 / 15)\end{array}$ & $\begin{array}{l}13 / 15 \\
(15 / 15)\end{array}$ \\
\hline Totals 68\% & $\begin{array}{l}38 / 45 \\
84 \%\end{array}$ & $\begin{array}{l}35 / 45 \\
78 \%\end{array}$ & $\begin{array}{l}36 / 45 \\
80 \%\end{array}$ & $\begin{array}{l}49 / 60 \\
82 \%\end{array}$ & $\begin{array}{l}35 / 45 \\
78 \%\end{array}$ & $\begin{array}{l}38 / 45 \\
84 \%\end{array}$ & $\begin{array}{l}40 / 45 \\
89 \%\end{array}$ \\
\hline Totals 95\% & $\begin{array}{l}42 / 45 \\
93 \%\end{array}$ & $\begin{array}{l}42 / 45 \\
93 \%\end{array}$ & $\begin{array}{l}41 / 45 \\
91 \%\end{array}$ & $\begin{array}{l}59 / 60 \\
98 \%\end{array}$ & $\begin{array}{l}44 / 45 \\
98 \%\end{array}$ & $\begin{array}{l}44 / 45 \\
98 \%\end{array}$ & $\begin{array}{l}45 / 45 \\
100 \%\end{array}$ \\
\hline
\end{tabular}

estimate accuracy are clearly illustrated where that latest dataset date does not contain the true event. In TS2 dataset MRRR1d run 3, for example, the latest date (MRRR5d) is too early at $68 \%$ confidence (1020$1160 \mathrm{cal} \mathrm{AD})$ and $95 \%$ confidence (990-1160 cal AD), and thereby the End Boundary and Last distributions generated by all models at $68 \%$ probability and the Last distributions generated by both exponential prior approaches at $95 \%$ probability are also too early (Table 12). No latest dataset dates are inaccurately late 
Table 33 Average precision of End Boundary HPD intervals generated by the exponential prior/modified Charcoal Outlier Model approach in main TS1 and TS2 models, in years. (summarised from Tables 7 and 14)

\begin{tabular}{llccr}
\hline Dataset IAT & \multicolumn{4}{l}{ Dataset size } \\
\cline { 2 - 5 } & 20 date & 15 date & 10 date & \multicolumn{1}{c}{ 5date } \\
\hline 10 years & $34(53)$ & $32(53)$ & $35(57)$ & $48(118)$ \\
50 years & $38(63)$ & $40(72)$ & $38(75)$ & $68(170)$ \\
100 years & $39(71)$ & $47(90)$ & $57(120)$ & $98(230)$ \\
200 years & $58(120)$ & $98(152)$ & $83(172)$ & $155(398)$ \\
500 years & $96(216)$ & $118(263)$ & $142(328)$ & $331(882)$ \\
\hline
\end{tabular}

Table 34 Precision of Last distributions generated by the exponential prior/modified Charcoal Outlier Model approach in main TS1 and TS2 models, in years (summarised from Tables 8 and 14)

\begin{tabular}{lllll}
\hline Dataset IA & \multicolumn{4}{l}{ Dataset size } \\
\cline { 2 - 5 } & $\mathbf{2 0}$ date & $\mathbf{1 5}$ date & $\mathbf{1 0}$ date & $\mathbf{5}$ date \\
\hline 10 years & $30(50)$ & $28(52)$ & $32(53)$ & $42(73)$ \\
50 years & $33(58)$ & $37(67)$ & $32(63)$ & $47(92)$ \\
100 years & $35(68)$ & $45(80)$ & $50(102)$ & $68(137)$ \\
200 years & $55(110)$ & $87(133)$ & $68(147)$ & $92(217)$ \\
500 years & $80(210)$ & $82(203)$ & $93(243)$ & $148(332)$ \\
\hline
\end{tabular}

at $95 \%$ probability in these theoretical studies, but in TS1 dataset M1e run 3 the latest date (M5e) is too late at $68 \%$ confidence (1260-1300 cal AD), and the End Boundary distributions generated by all models from this dataset are also too late at $68 \%$ probability (Table 4). Indeed, the End Boundaries generated by both uniform prior/Charcoal Outlier Models from this dataset are also too late at $95 \%$ probability, and these are the only two inaccurate End Boundary HPD intervals at $95 \%$ probability in TS1.

The data associated with these examples also illustrate how model specification can mitigate against latest date variation, since the earlier distributions generated by models with exponential priors are generally more accurate where the latest dataset date is relatively late, whilst the older estimates generated by models incorporating the Charcoal Outlier Model are generally more accurate where the latest dataset date is relatively early. In TS2 dataset MRRR1c run 3 , for example, the latest simulated date contains the true event at 95\% confidence (1220-1380; MRRR1c) but is too late at $68 \%$ confidence $(1260-1300$ cal AD; MRRR1c; Additional file 2: Sect. 2.9.10); and in this instance both Charcoal Outlier modelling approaches have generated inaccurately late End Boundaries at $68 \%$ probability, and only the exponential/no outlier approach has generated an accurate End Boundary at $68 \%$ probability. In contrast, the only two inaccurate End Boundaries at $95 \%$ probability in TS2 are too early (MR1c run 3 and MRR1d run 1) and, predictably therefore, these distributions are associated with comparatively early dataset latest dates and exponential prior/no outlier modelling approaches.

These processes also have implications for distribution selection; since the earlier Last distributions are often more accurate than End Boundaries where a comparatively late latest date pertains (e. g. TS2, MR1b run 2, exponential prior/no outlier) while End Boundaries are more accurate where an early latest date pertains. Indeed, given the preponderance of relatively early dates, this might explain the comparatively greater consistency of End Boundary distributions overall. The estimates generated from the TS2 dataset MRR1d run 1 illustrate how these processes affect both distribution and model selection, since the latest date (MRR3d) contains the true date at 95\% confidence (1040-1270 cal AD) but is too early at $68 \%$ confidence $(1050-1230 \mathrm{cal}$ AD) (Table 11), and thereby: the End Boundary generated by the exponential prior/no outlier modelling approach is too early at $95 \%$ probability; the End Boundaries generated by both exponential prior models are too early at $68 \%$ probability; the Last distributions generated by both exponential prior models are also too early at $95 \%$ probability; and the Last distributions generated by all three modelling approaches are too early at $68 \%$ probability (Table 12). The uniform prior/Charcoal Outlier Model approach, however, has generated accurate End Boundary estimates at both $95 \%$ and $68 \%$ probability from this high IAt dataset, as well as an accurate Last distribution at $95 \%$ probability.

Ultimately, the Last and End Boundary distributions generated by different model specifications form a continuous chronological spectrum: from the very early and precise Last distributions generated from large low IAt datasets by the exponential prior/ no outlier modelling approach; to the later and more imprecise End Boundary distributions generated from small high IAt datasets by the uniform prior/Charcoal Outlier Model approach. The evidence presented in TS2 also suggests this spectrum correlates with the accuracy of estimates generated from datasets subject to different IAt/size characteristics: wherein the exponential prior/no outlier approach has generated the most consistently accurate Last distributions from datasets with an IAt which is lower than 100 years (where latest dates are likely to be relatively late), and 
the uniform prior/Charcoal Outlier approach has generated the most consistently accurate Last distributions from datasets of 100 years IAt and above (where latest dates are likely to be relatively early) (Table 13). Unsurprisingly, given their precision, the proximity of the Last distribution median values to the true event date follows this same pattern (Table 15), while the accuracy threshold between these contrasting approaches is slightly broader in the End Boundary distribution evidence. Both exponential prior approaches present the most consistently accurate End Boundary distributions from datasets with an IAt lower than 100 years in TS1 and TS2, and approaches which include a Charcoal Outlier Model are more consistently accurate from 200 years IAt and above (although this breaks down at 500 years IAt in TS1) (Table 31). That a considerable overlap between these theoretical Last and End Boundary spectra also pertains is clearly illustrated in the TS2 results, wherein the most consistently accurate End Boundaries have been generated by the exponential prior/Charcoal Outlier Model approach, whilst the most consistently accurate Last distributions have been generated by the uniform prior/Charcoal Outlier Model approach. Indeed, both approaches have generated accurate estimates from all datasets at $95 \%$ probability and $87 \%$ of all models at $68 \%$ probability, while closely comparable average precision and median values between these different distributions confirms they overlap considerably (Tables 13, 15 and 17).

These results are consistent with those presented by previous authors. If the $500 \mathrm{yrs}$ IAt datasets are disregarded, then the uniform prior/Charcoal Outlier Model approach does indeed generate more consistently accurate End Boundary distributions than the exponential prior/no outlier approach [17], with this latter approach once again returning some precise but inaccurate End estimates at $95 \%$ probability (Table 16). These inaccuracies are limited to datasets above 50 years IAt, however, and the End Boundary estimates generated by the exponential prior/Charcoal Outlier Model approach are more consistently accurate overall. In the above studies this is even evident where dataset IAt range is much reduced, and most particularly so with lower IAt datasets where the less accurate and less precise uniform prior approach has been specified. These data, therefore, also support previous MERLF analysis protocols which had promoted a binary short-lived (exponential prior) and long-lived (Charcoal Outlier Model) approach [7]; but allows an increased role for exponential prior model specifications and further understanding of how these relate to Combine and Charcoal Outlier Model approaches. The accuracy of the Combine distributions generated from 10 years IAt datasets is also resonant of Waterbolk's (1971) Group A samples which, he suggested, would extend up to 20 years [10]. Ultimately, these theoretical results provide a less binary Bayesian framework which can inform our interpretations of the datasets returned by MERLF materials from the six Scottish medieval case study buildings.

\section{The case studies}

The compositions presented by these case study MERLF assemblages confirm that a range of different locally available tree taxa were exploited for limekiln fuel in Scotland during this period, and the maximum age ranges presented by the resulting radiocarbon datasets are generally consistent with the taxa-specific and habitat-contingent IAt of those woodland

Table 35 Case study MERLF assemblage and radiocarbon dataset character. (from Tables 16, 18, 20, 22, 24,26, Appendix 1: Table 37).

\begin{tabular}{|c|c|c|c|c|c|c|}
\hline Case study & Site and building name & Dataset size & MERLF taxa & $\begin{array}{l}5 \% \text { statistical } \\
\text { consistency }\end{array}$ & $\begin{array}{l}\text { Combine } \\
\text { agreement }\end{array}$ & Age range $95 \%$ (years) \\
\hline CS1 & $\begin{array}{l}\text { Castle Fincharn } \\
\text { main block }\end{array}$ & 5 & Corylus & Pass & Good & -30 to 220 \\
\hline CS2 & $\begin{array}{l}\text { Aros Castle } \\
\text { north-west block }\end{array}$ & 5 & Betula Corylus & Fail & Poor & 35 to 190 \\
\hline CS3 & $\begin{array}{l}\text { Castle Roy } \\
\text { enclosure and tower }\end{array}$ & 5 & Betula Pinus & Pass & Poor & -75 to 210 \\
\hline CS4 & $\begin{array}{l}\text { Lochindorb Castle } \\
\text { primary enclosure }\end{array}$ & 5 & Quercus & Fail & None & 1555 to 1925 \\
\hline CS5 & $\begin{array}{l}\text { Achanduin Castle } \\
\text { enclosure and hall }\end{array}$ & 3 & Betula Quercus & Pass & Good & O to 155 \\
\hline CS6 & $\begin{array}{l}\text { Lismore Cathedral } \\
\text { nave }\end{array}$ & 3 & Quercus/Corylus & Fail & Poor & 125 to 345 \\
\hline $\mathrm{CS}^{*}$ & $\begin{array}{l}\text { Lochindorb Castle } \\
\text { reduced dataset }\end{array}$ & 4 & Quercus & Pass & Poor & $10-220$ \\
\hline
\end{tabular}


sources. This includes: the radiocarbon dataset associated with the Corylus sp. dominated assemblage from Castle Fincharn, which generated an age range of -30 to 220 years consistent with a 5 date mean lifespan of 10 years IAt or less; the dataset associated with the Betula sp. dominated assemblage from Castle Aros which generated an age range of 35 to 190 years consistent with a 5 date mean lifespan of 50 years IAt or less; and the smaller dataset associated with the Quercus sp. dominated assemblage from Lismore Cathedral nave, which nevertheless generated an age range of 125 to 345 years consistent with a 5 date mean lifespan of 200 years IAt or less (Table 35).

The association of some taxa or environments with comparatively high mean lifespans does not of course preclude the exploitation of shorter-lived or immature wood, where growth habits and woodland population dynamics allow. Indeed, although the case study results presented here are biased by an analysis strategy which privileged the selection of shorter-lifespan taxa, it is evident from the statistical consistency and relatively narrow age ranges presented by the mixed assemblages from Castle Roy (CS3) and Achanduin Castle (CS5) that short-lived fragments of long life-span taxa were also included in limekiln charges (Table 35 ). The only case study dataset which generated an age range broader than expected is associated with the Quercus sp. dominated assemblage from Lochindorb Castle, which returned a dataset age range (1555 to 1925 years) consistent with a 5 date mean lifespan of over 500 years IAt. This is improbable, and when the single very early radiocarbon determination (SUERC-75752) is manually excluded from the model, the age range of the remaining dataset $(10-220$ years; Additional file 3: Sect. 3.37) is consistent with a 5 date dataset of 50 years IAt or less.

Pre-existing historical, architectural, and archaeological evidence has situated initial building construction at the six case study sites in the same long 13 th-century period, and within chronological periods ranging between 18 and 122 years (Table 36). The relationships between this evidence and the masonry buildings under consideration are indirect and open to challenge, and these periods are much broader than the true event date from which the simulated datasets were generated in TS1 and TS2. The lack of terminal ring evidence in five of the case study assemblages, moreover, introduces a bridging period between these datasets and the constructional date which does not apply to those theoretical studies. However, all case study datasets contain at least one latest date which is consistent with the pre-existing evidence from other disciplines, five of the six present latest dataset dates which extend past their potential historical TAQs, and none are inconsistently late (Table 36 ). This evidence suggests the volume of material missing material from these MERLF fragments (and so the extent of that bridging period) is quite limited and, although small dataset sizes limit the interpretive potential of their unmodelled distributions (Figs. 5, 7, 9, 11, 13, $15)$, these case study datasets generally contain high fractions of consistent dates.

As in both main theoretical studies, the Last and End Boundary distributions generated from these case study datasets present continuous chronological spectra: from the earliest and most precise Last distributions generated using the exponential prior/no Outlier approach; to the later and broader distributions generated using the uniform prior/Charcoal Outlier Model (Tables 14, 16. 18, 20, 22, 24). It is notable that modifying Charcoal Outlier Model time-constants to reflect the mean lifespans of the dominant taxa has increased Last and End Boundary precision in all six case studies and, in line with its increased effect on less precise distributions in TS1, this probably reflects the smaller size of these case study datasets. An overlap between the latest Last distributions and earliest End Boundary is again evident in these studies, and this is very clearly illustrated in overlapping estimateTPQ/TAQ percentages.

The sum of these estimate-TPQ-TAQ percentages reflects contrasts in the precision of these different types of evidence, and these vary from Castle Fincharn (whose high sum percentages reflect a low IAt Corylus sp. dominated MERLF radiocarbon dataset and the moderate precision of the pre-existing documentary evidence relating to the wider site) and Lochindorb Castle and Lismore Cathedral nave (which are both associated with relatively high age range Quercus sp. dominated MERLF radiocarbon datasets). Except for the estimate generated by the uniform prior/Charcoal Outlier Model approach to the Lismore Cathedral nave dataset, all Last and End Boundary distributions generated by these different model specifications at $95 \%$ probability are consistent with the other evidence relating to these sites, and the proximity of some of these estimate-TPQ-TAQ probability sums to $200 \%$ indicates that these Bayesian estimates are closely consistent with that evidence. The consistency of this evidence usefully suggests that the masonry buildings from which these MERLF samples were removed can be reasonably associated with the wider evidence relating to these sites, and where late latest dates have defined comparatively late lower estimate limits (e. g. Aros Castle NW block and Lismore Cathedral nave) then considerable gains in 


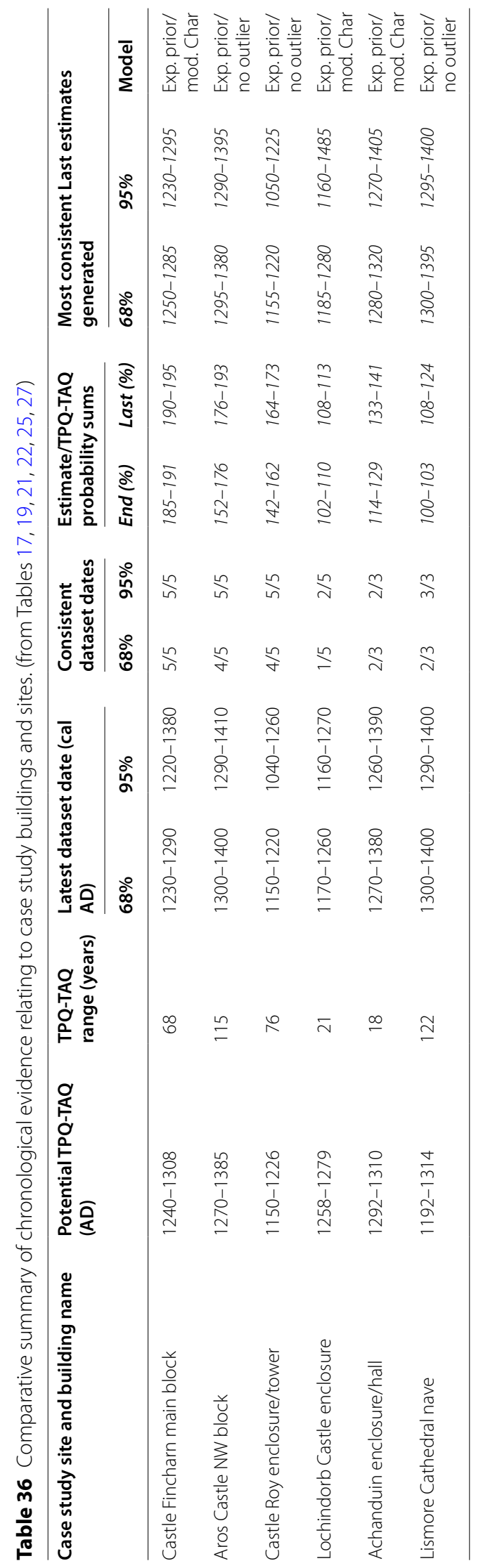


multidisciplinary interpretation precision have been made. It is salient that the datasets associated with both of these sites are high IA and statistically inconsistent at $5 \%$ probability, however, and therefore the End Boundary distributions generated from these data are relatively broad.

Recognising how we might retain accuracy while maximising precision through sample selection and model specification requires further comparison with the theoretical and ecological data. Variation in dataset age ranges and levels of statistical consistency across these studies suggests that the assemblages did include materials subject to some IA, and only two of these case study datasets have presented good Combine agreement indices (even including the reduced Lochindorb Castle CS4* dataset) (Table 35). That Combine distributions could be generated at all suggests the IA associated with five of these assemblages is reasonably limited, however, and comparison with the theoretical data from TS1 and TS2 suggests four of the six case studies (or five if the reduced Lochindorb Castle is included) present dataset age ranges consistent with mean lifespans associated with less than 50 years IAt. Importantly, this situates these four-five studies below the considerable reduction in estimate precision associated with theoretical datasets subject to IAts of over 100 years in TS1 and TS2 (Tables 32 and 34), and all (100\%) End Boundary and Last distributions generated from such narrowly distributed 5 date datasets were consistently accurate in TS2 at both $95 \%$ and $68 \%$ probability (Table 13 ). The estimate/TPQ-TAQ sum percentages also illustrate that interdisciplinary consistency generally increases with precision, and the most consistent estimates across all six case studies were Last distributions generated by exponential prior modelling specifications. Indeed, in most cases these Last distributions are very similar to each available latest dataset date, and constructional dates close to the lower limits of these distributions are often most convincing.

The association of higher IAt and small datasets with earlier latest dates and decreased fractions of accurate dates in TS1 and TS2 (Table 28) suggests that it would be prudent to include a Charcoal Outlier Model within model specifications where radiocarbon datasets are less narrowly distributed, and yet the botany suggests different approaches to the case study evidence are probably required. Both Quercus sp. dominated case study assemblages (Lochindorb Castle enclosure and Lismore Cathedral nave) are statistically inconsistent at $5 \%$ significance, but the Lochindorb dataset contains an extremely early determination, high age range, and low fraction of consistent dates. This assemblage is completely dominated by Quercus sp. samples, and although manual exclusion of the early determination has decreased dataset IAt considerably, this still contains only two dates which are consistent with historical evidence at 95\% probability and the latest date does not extend beyond the documentary TAQ at either $68 \%$ or $95 \%$ probability (Appendix 1: Table 37). Notably, this reduced dataset is also the only example to generate End Boundary estimates which are more consistent with the historical evidence than the earlier and more precise Last distributions, and the End Boundary generated by the exponential prior/modified Charcoal Outlier Model approach to the dataset is the most consistent overall (Appendix 1: Table 38). Archaeobotanical and statistical evidence suggests this approach is less relevant to the Lismore Cathedral nave study since, although the high age range associated with this small dataset is largely predicated on residuality in two early Quercus sp. fragments, the latest date is associated with a fragment of Corylus. Since the uniform prior/Charcoal Outlier Model approach to this dataset has generated the only inconsistent estimate in the data from all six studies (Table 27), it is entirely possible that the calibrated radiocarbon date associated with this latter fragment is relatively late. This latest determination allows the 200 years or less IAt of the wider Quercusdominated radiocarbon dataset and its relationship to the constructional event to be interpreted with greater confidence. Notably, this latest radiocarbon determination also calibrates to a date almost identical to the Last distribution generated from the wider dataset using the (most precise) exponential prior/no Outlier approach, and this currently represents the most convincing estimate for the construction of this fabric. The correlation between dataset IAt and latest date does not hold for the radiocarbon evidence returned by this mixed-taxa assemblage, and the application of a Charcoal Outlier Model approach to this very small dataset is inappropriate.

\section{Conclusion}

This paper has presented further evidence that a range of different tree taxa were exploited for limekiln fuel in Scotland during the medieval period, and the range of MERLF taxa surviving from this process are generally consistent with regional phytogeographic 
distributions. The samples selected from these assemblages for radiocarbon analysis have returned datasets characterised by an array of different age range distributions and most of these are associated with some level of IA. At this stage in the research cycle, however, the IAt of these materials does appear to be constrained by the taxa-specific and habitat-contingent lifespans and post-mortem durabilities of the parent wood fuels. Given that shorter lifespan taxa were generally selected for radiocarbon analysis where possible, it seems probable that the carbon distributions in these assemblages are generally equivalent to the available woodland source. MERLF assemblages can therefore be considered an excellent source of palaeoenvironmental information, with a research potential again underscored by the stratigraphically secure mortar material within which these materials have been entrapped.

The MERLF assemblages considered in this paper are dominated by wood-charcoal fragments without surviving terminal ring evidence, and the range of radiocarbon determinations returned by selected samples suggests that calibrated dates from single determinations, unweighted mean averages of multiple determinations and/or bulk samples, cannot be accepted as direct evidence for the construction of masonry buildings without other forms of evidence. The TPQ role performed by such determinations can be of considerable value for multidisciplinary interpretation, particularly where the radiocarbon evidence is relatively late and documentary evidence is convincing and early. Increasing the potential for these buildings and materials to inform interdisciplinary (rather than multidisciplinary) discourse, however, requires accurate standalone constructional estimates of greater precision.

In the absence of non-residual intrusive materials, the generation of accurate Last and End Boundary distributions from MERLF radiocarbon datasets subject to significant IA relies more on the accuracy of the latest available determination, than on dataset IAt, dataset size, or model specification. Last and End Boundary precision, however, is very closely related to all three of these parameters; decreasing with increasing dataset $\mathrm{IA \tau}$, decreasing dataset size, and model specifications which include uniform priors or Charcoal Outlier Models. These factors are interrelated and can be cumulative, and the Last and End Boundary distributions generated by different model specifications thereby present continuous and overlapping spectra. These range from the early and precise Last distributions generated from large low-IAt datasets by models specified with an exponential prior/ no outlier approach; to the later and less precise End Boundary distributions generated from small highIAt datasets by models specified with uniform priors and the default Charcoal Outlier Model.

Different Bayesian model specifications can therefore be imposed on MERLF radiocarbon data to maximise precision whilst retaining accuracy. The data relating to the 13th-century events presented in this paper suggests that: (i) Where the IAt of a dataset is limited in 10 years or less, then determinations are likely to be statistical consistent at $5 \%$ significance and a Combine average distribution is likely to represent an accurate and very precise constructional estimate; (ii) Where the IAt of a dataset is limited to 50 years or less, then determinations are unlikely to be statistical consistent at $5 \%$ significance and Combine agreement indices will be poor, but the Last distribution generated by a model specified with exponential priors is likely to represent an accurate and reasonably precise constructional estimate; and (iii) Where the IAt of a dataset is greater than 100 years, then a Last distribution generated by a model with a Charcoal Outlier Model is likely to generate an accurate but imprecise constructional estimate, while modification of the outlier time-constant is likely to increase precision where dataset size is limited.

The studies considered in this paper provide further evidence that Bayesian techniques can generate consistently accurate constructional estimates for medieval masonry buildings from MERLF radiocarbon data, whatever the ecological provenance of the limekiln fuel source. Estimate precision is contingent upon source ecology but can be increased by a more informed approach to materials analysis and interpretation. The radiocarbon evidence considered here and elsewhere $[7,76]$ is biased by the selection of single entity MERLF fragments from shorter lifespan tree taxa, where possible, and most of these have returned determinations consistent with (i) and (ii) above. It seems likely this has enabled the generation of more precise constructional estimates, although in many cases precision might be further increased by expanding these radiocarbon datasets to include higher precision (reduced error margin) analysis of short lifespan MERLF fragments.

\section{Appendix 1}

CS4* - Lochindorb Castle Phase 1 (*with reduced dataset manually excluding determination SUERC-75752)

See Tables 37 and 38; Figures 17 and 18. 


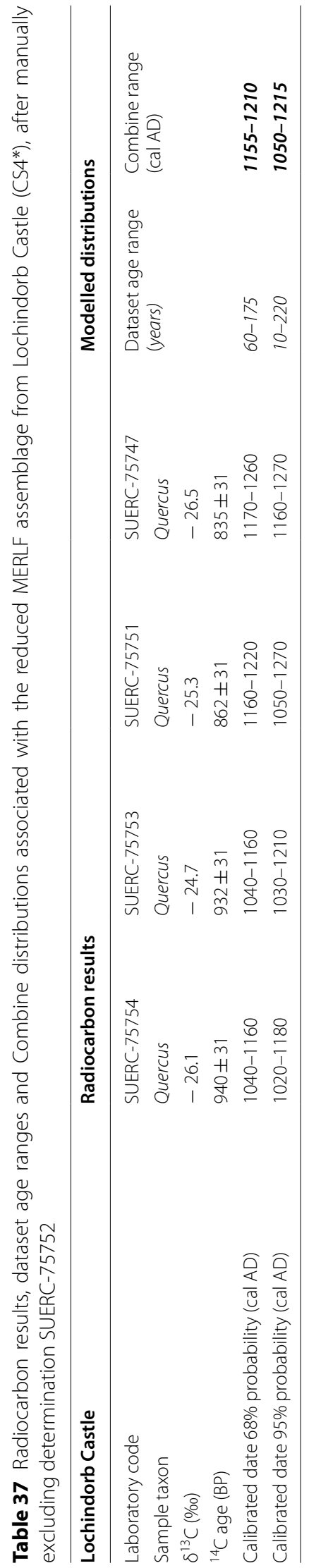




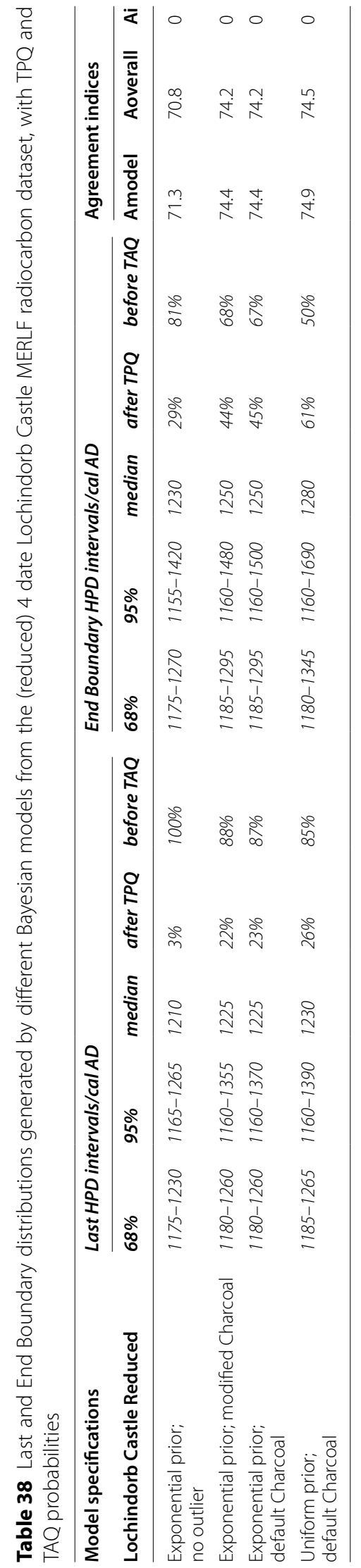




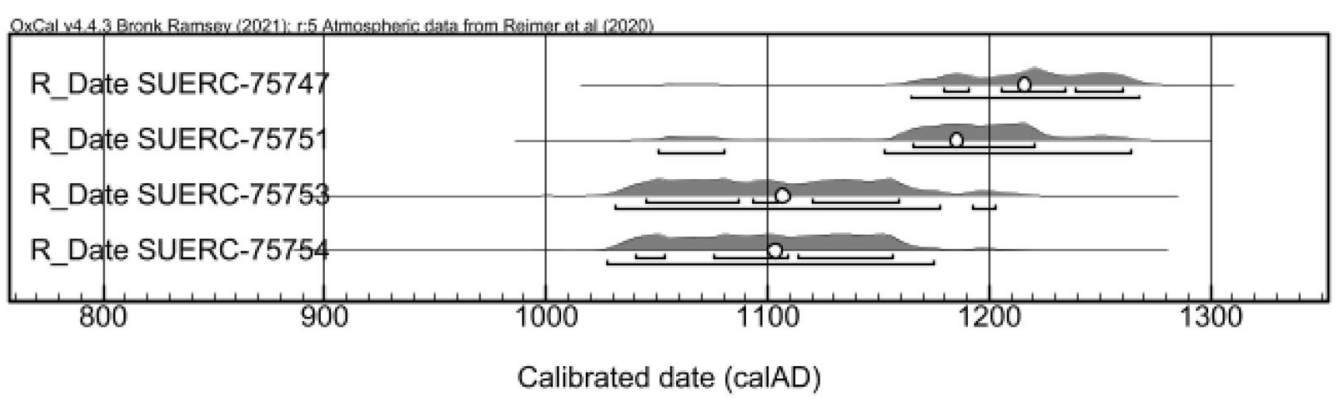

Fig. 17 Unmodelled calibrated probability distributions associated with four MERLF samples removed from the phase 1 enclosure of Lochindorb Castle. Small circles represent the mean average of each distribution

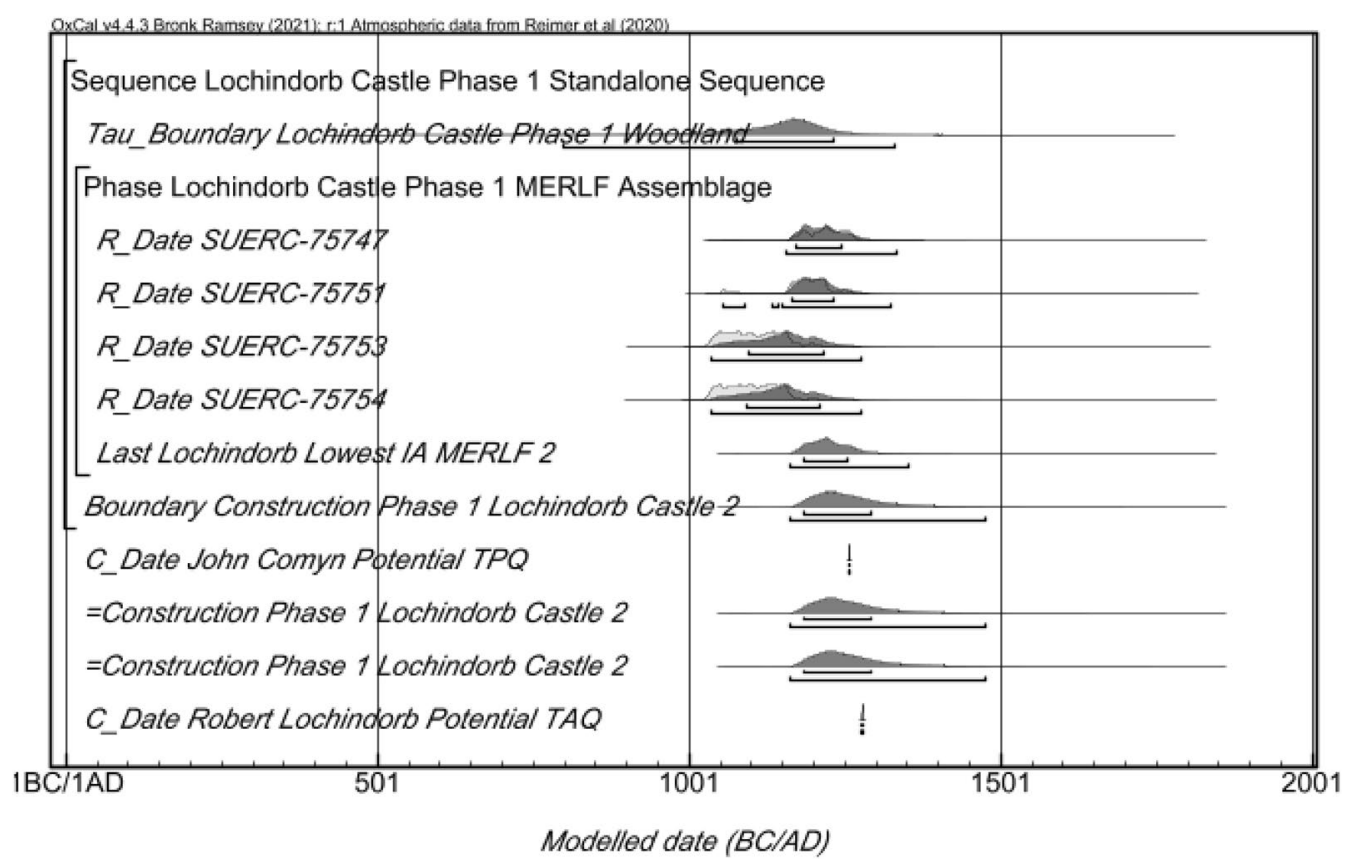

Fig. 18 Probability distributions generated from the reduced Lochindorb Castle dataset. All four radiocarbon determinations have been situated within a single phase with a Tau Start Boundary, and all four tagged with a 100\% Outlier Probability within a Charcoal Outlier Model with a 300 year time-constant

\section{Abbreviations}

CS: Case Study; ESM: Electronic Supplementary Material; HPD: Highest Posterior Density; IA: Inbuilt Age; IAt: Mean Dataset Inbuilt Age; MERLF: MortarEntrapped Relict Limekiln Fuel; TAQ: terminus ante quem (Limit before which); TPQ: terminus post quem (Limit after which); TS: Theoretical Study.

\section{Supplementary Information}

The online version contains supplementary material available at https://doi. org/10.1186/s40494-021-00568-3.

Additional file 1. Theoretical Study 1 (TS1)

Additional file 2. Theoretical Study 2 (TS2).

Additional file 3. Case Studies 1-6 (CS1-6+ CS4*).

\section{Acknowledgements}

The data reconsidered in this paper was generated during PhD research undertaken at the University of Edinburgh and post-doctoral research at the University of Stirling. Within these projects, charcoal identification was undertaken with Dr Mike Cressey (then at CFA Archaeology) and radiocarbon analysis was funded by Historic Environment Scotland. The author is grateful for the comments provided by two anonymous peers and the journal editor, which improved this paper significantly, and for their patience when the original manuscript was withdrawn in 2020 following the emergence of the new calibration curve.

\section{Authors' contributions}

This paper is the work of MT, who is sole author.

Funding

Not applicable.

Availability of data and materials

All the data relating to this paper are included in the main text and additional file. 


\section{Declarations}

\section{Competing interests}

The authors declare that they have no competing interests.

Received: 17 May 2021 Accepted: 25 July 2021

Published online: 16 September 2021

\section{References}

1. Gourdin W, Kingery W. The beginnings of pyrotechnology: Neolithic and Egyptian lime plaster. J Field Archaeol. 1975;2:133-50.

2. Kingery W, Vandiver P, Prickett $M$. The beginnings of pyrotechnology, part II: production and use of lime and gypsum plaster in the pre-pottery Neolithic near-east. J Field Archaeol. 1988;15(2):219-34.

3. Friesem D, Abadi I, Shaham D, Grosman L. Lime plaster cover of the dead 12000 years ago - new evidence for the origins of lime plaster technology. Evol Hum Sci. 2019;1:e9.

4. Carò F, Riccardi M, Mazzilli SM. Characterization of plasters and mortars as a tool in archaeological studies: the case of Lardirago Castle in Pavia, northern Italy. Archaeometry. 2008;50:85-100.

5. Elsen J, Mertens G, Van Balen K. Raw materials used in ancient mortars from the Cathedral of Notre-Dame in Tournai (Belgium). Eur J Mineral. 2011;23:871-82

6. Elsen J. Microscopy of historic mortars-a review. Cem Concr Res. 2006;36:1416-24.

7. Thacker M. Dating medieval masonry buildings by radiocarbon analysis of mortar-entrapped relict limekiln fuels - a buildings archaeology. J Archaeol Method Theory. 2020. https://doi.org/10.1007/ s10816-020-09444-z.

8. Boaretto E. Dating materials in good archaeological contexts: the next challenge for radiocarbon analysis. Radiocarbon. 2009;51(1):275-81.

9. Bayliss A, Bronk Ramsey C, van der Plicht J, Whittle A. Bradshaw and Bayes: towards a timetable for the Neolithic. Camb Archaeol J. 2007:17:1-28.

10. Waterbolk H. Working with radiocarbon dates. Proc Prehist Soc. 1971;37:15-33.

11. Warner R. A proposed adjustment for the "old-wood effect". In: Mook W, Waterbolk H (editors). In: Proceedings of the 2nd Symposium of 14C \& Archaeology, Groningen 1987. 1990, p.159-72.

12. Bowman S. Radiocarbon Dating. London: British Museum Publications; 1990. p. 50-1.

13. Bronk RC. Dealing with outliers and offsets in radiocarbon dating. Radiocarbon. 2009;51(3):1023-45.

14. Dean J. Independent dating in Archaeological Analysis. Adv Archeol Method Theory. 1978;1:223-55.

15. McFadgen B. Dating New Zealand archaeology by radiocarbon. NZ J Sci. 1982:25:379-92.

16. Dee M, Bronk Ramsey C, Shortland A, Higham T, Rowland J. Reanalysis of the chronological discrepancies obtained by the old and middle kingdom monuments project. Radiocarbon. 2009;51(3):1061-70.

17. Dee M, Bronk RC. High-precision bayesian modeling of samples susceptible to inbuilt age. Radiocarbon. 2014;56(1):83-94

18. Ashmore P. Radiocarbon dating: avoiding errors by avoiding mixed samples. Antiquity. 1999;73:124-30.

19. van Balen K. Understanding the lime cycle and its influence on historical construction practice. In: Huerta S, editor. In: Proceedings of the first international congress on construction history, Madrid, 20th-24th January 2003, Madrid.

20. Enquist B, West $G$, Charnov E, Brown J. Allometric scaling of production and life-history variation in vascular plants. Nature. 1999:401:907-11.

21. van Gelder H, Poorter L, Sterck F. Wood mechanics, allometry and lifehistory variation in a typical rain forest tree community. New Phytol. 2006;171(2):367-78

22. Marbà N, Duarte C, Agusti S. Allometric scaling of plant life history. PNAS. 2007;104(40):15777-80.

23. Black B, Colbert J, Pederson N. Relationships between radial growth rates and lifespan within North American tree species. Ecoscience. 2008;15(3):348-57.
24. Johnson S, Abrams M. Age class, longevity and growth rate relationships: protracted growth increases in old trees in the eastern United States. Tree Physiol. 2009;29:1317-28.

25. Biondi F. From dendrochronology to allometry. Forests. 2020;11(2):146.

26. Brown P. OLDLIST: a database of maximum tree ages. In: J Dean, D Meko, T Swetnam, editors. In: Tree rings, environment, and humanity: proceedings of the international conference, Tucson, Arizona, 17-21 May, 1994. Radiocarbon 1996, p. 727-31.

27. Shorohova E, Kapitsa E. Influence of the substrate and ecosystem attributes on the decomposition rates of coarse woody debris in European boreal forests. For Ecol Manage. 2014;315:173-84.

28. Gavin D. Estimation of Inbuilt Age in radiocarbon ages of soil charcoal for fire history studies. Radiocarbon. 2001;43(1):27-44.

29. Di Filippo A, Pederson N, Baliva M, Brunetti M, Dinella A, Kitamura K. Knapp H, Schirone B, Piovesan G. The longevity of broadleaf deciduous trees in Northern Hemisphere temperate forests: insights from tree-ring series. Front Ecol Evol 2015; 3, article 46: 1-15.

30. Rackham O. Ancient Woodland: its history, vegetation and uses in England. Kirkcudbrightshire: Castlepoint Press; 2003

31. Mitchell A. A field guide to the trees of Britain and Northern Europe. London: Collins; 1974

32. Cameron A. Managing birch woodlands for the production of quality timber. Forestry. 1996;69(4):357-71.

33. Coppins A \& Coppins B. Atlantic Hazel. Scottish Natural Heritage: Edinburgh; 2010.

34. McVean D. Woodland and Scrub. In: J Burnett, editor. The Vegetation of Scotland. Oliver \& Boyd: Edinburgh \& London; 1964, pp 144-67.

35. Jahn G. Temperate deciduous forests of Europe. In: Röhrig E, Ulrich B, editors. Ecosystems of the world 7: temperate deciduous forests; 1991, pp 377-502. Amsterdam: Elsevier. fig 13.12E.

36. Stephenson $\mathrm{N}$, et al. Rate of tree carbon accumulation increases continuously with tree size. Nature. 2014;507:90-3.

37. Jenkins J, Chojnacky D, Heath L, Birdsey R. National-scale biomass estimators for United States tree species. For Sci. 2003;49(1):12-35.

38. Harmon M, Franklin J, Swanson F, Sollins P, Gregory S, Lattin J, Anderson N, Cline S, Aumen N, Sedell J, Lienkaemper G, Cromack K, Cummins K. Ecology of coarse woody debris in temperate ecosystems. Adv Ecol Res. $1986: 15: 133-302$

39. Manning S, Birch J, Conger M, Dee M, Griggs C, Hadden C, Hogg A, Bronk Ramsey C, Sanft S, Steier P, Wild E. Radiocarbon re-dating of contact-era Iroquoian history in north-eastern North America. Sci Adv. 2018. https:// doi.org/10.1126/sciadv.aav0280.

40. Ranius T, Niklasson M, Berg N. Development of tree hollows in pedunculate oak (Quercus robur). For Ecol Manage. 2009;257(1):303-10.

41. White J. Forest and woodland trees in Britain. Oxford: Oxford University Press; 1995. p. 130.

42. Nicholls $\mathrm{G}$, Jones M. Radiocarbon dating with temporal order constraints. J R Stat Soc Ser C Appl Stat. 2001;50:503-21.

43. Berger R. 14C Dating Mortar in Ireland. Radiocarbon. 1992:34(3):880-9.

44. Berger R. Radiocarbon dating of early medieval Irish monuments. In: Proceedings of the royal Irish academy. Section C: Archaeology, Celtic Studies, History, Linguistics, Literature 95C 1995;(4): 159-74.

45. Dee M, Wengrow D, Shortland A, Stevenson A, Brock F, Flink L, Bronk RC. An absolute Chronology for early Egypt using Radiocarbon dating and Bayesian Statistical Modelling. Proc R Soc A. 2013:469:20130395. https:// doi.org/10.1098/rspa.2013.0395.

46. van Strydonck M, van Der Borg K, de Jong A, Keppens E. Radiocarbon dating of lime fractions and organic material from buildings. Radiocarbon. 1992;34(3):873-9.

47. Rutgers $L$, de Jong $A$, van der Borg K. Radiocarbon dates from the Jewish catacombs of Rome. Radiocarbon. 2002;44(2):541-7.

48. Tubbs L, Kinder T. The use of AMS for the dating of lime mortars. Nucl Instrum Methods Phys Res. 1990;B52:438-41.

49. Thacker M. The Castle of Achanduin, Lismore - a point of reference for the radiocarbon analysis of mortar-entrapped relict limekiln fuels. Radiocarbon. 2020:62(6):1563-75. https://doi.org/10.1017/RDC.2020.57.

50. Ward G, Wilson S. Procedures for comparing and combining radiocarbon age determinations: a critique. Archaeometry. 1978;20:19-32.

51. Bronk RC. Bayesian analysis of radiocarbon dates. Radiocarbon. 2009:51(1):337-60. 
52. Thacker M. Castle Camus, Isle of skye-buildings, materials \& radiocarbon analysis in the borderlands of medieval sleat. Proc Soc Antiquaries Scotland. 2020;149:277-301. https://doi.org/10.9750/PSAS.149.1298.

53. Reimer, et al. The IntCal20 Northern Hemisphere radiocarbon age calibration curve (0-55 cal kBP). Radiocarbon. 2020;62(4):725-57.

54. BSI. Durability of wood and wood-based products - Natural durability of solid wood - Part 2: guide to natural durability and treatability of selected wood species of importance in Europe. British Standards Institution: London; 2017

55. Bronk RC. Radiocarbon calibration and analysis of stratigraphy: the OxCal program. Radiocarbon. 1995;37(2):425-30.

56. Bayliss A. Anglo-Saxon graves and grave goods of the 6 th and 7 th centuries ad: a chronological framework. 2017. Routledge: Oxford. p. 85-6.

57. Walters S, Betula L. in Britain. Proc Bot Soc Br Isles. 1968;7:179-80.

58. Gimingham C. Ecological aspects of Birch. Proc R Soc Edinburgh Sect B Biol Sci. 1984;85(1-2):65-72.

59. Pelham J, Gardiner A, Smith R, Last F. Variation in Betula pubescens Ehrh. (Betulacae) in Scotland: its nature and association with environmental factors. Bot J. 1988;96(3):217-34.

60. Atkinson M. Betula Pendula Roth (B. Verrucosa Ehrh.) and B. Pubescens Ehrh. J Ecol. 1992;80(4):837-70.

61. Edwards C, Mason W. Stand structure and dynamics of four native scots pine (Pinus sylvestris L.) woodlands in northern Scotland. Forestry. 2006;79(3):261-77.

62. Mencuccini M, Oñate M, Peñuelas J, Rico L, Munné-Bosch S. No signs of meristem senescence in old scots pine. J Ecol. 2014;102:555-65.

63. Stell G, Bailie M. The great hall and roof of Darnaway Castle, Moray. In: Sellar W, editor. Moray: Province and People. The Scottish Society for Northern Studies: Edinburgh; 1993. p. 163-86.

64. Crone A, Mills C. Timber in Scottish Buildings, 1450-1800-a dendrochronological perspective. Proc Soc Antiqu Scotl. 2012;142:329-69.

65. Wilson S. Native Woodlands of Scotland. Edinburgh: Edinburgh University Press; 2015. p. 133.

66. Thacker M. Constructing Lordship in North Atlantic Europe: the archaeology of masonry mortars in the medieval and later buildings of the
Scottish North Atlantic. 3 Vols. Unpublished PhD. thesis. University of Edinburgh; 2016

67. Thacker M. MacGillechrist's Castle: an environmental study in medieval buildings archaeology from Argyll. In: P Martin, editor. Castles and Galleys. A re-assessment of the historic galley-castles of the Norse-Gaelic seaways. IBT: Laxay; 2017. p. 157-71.

68. Thacker M. The medieval castle of Dun Aros: buildings archaeology and chronological consistency on the shores of the Sound of Mull. In: Proceedings of the Society of Antiquaries of Scotland 2021, Forthcoming

69. Thacker M. Castle Roy: investigating medieval cultural landscapes, buildings and materials in Badenoch, Abernethy and Mar. Int J Architect Herit. 2020. https://doi.org/10.1080/15583058.2020.1745321.

70. Thacker M. An Ecology of Castle Construction: geoarchaeology, archaeobotany and radiocarbon analysis in the ecotone of Lochindorb Castle. In: RILEM PRO 130; Proceedings of the 5th Historic Mortars Conference, Pamplona. RILEM publications: Paris; 2019, p. 808-26.

71. Caldwell D, Stell G, Turner D. Excavations at Achanduin Castle, Lismore, Argyll, 1970-5: findings and commentary. Proc Soc Antiqu Scotl. 2015;145:349-69.

72. Thacker, M. Lismore Cathedral. Discovery \& Excavation in Scotland; 2019.

73. Thacker in prep. Materials Analysis at Lismore Cathedral [working title].

74. Brown A, Duncan A. The cathedral church of Lismore. Trans Scot Ecclesiol Soc. 1957;15(1):41-55.

75. Fawcett R. The Architecture of the Scottish Medieval Church. New Haven \& London: Yale University Press; 2011.

76. Thacker M. Medieval buildings \& environmental change: chronology, ecology \& political administration at Castle Sween, Knapdale. Archaeol Anthropol Sci. 2020;12:238. https://doi.org/10.1007/s12520-020-01162-7.

\section{Publisher's Note}

Springer Nature remains neutral with regard to jurisdictional claims in published maps and institutional affiliations.

\section{Submit your manuscript to a SpringerOpen ${ }^{\circ}$ journal and benefit from:}

- Convenient online submission

- Rigorous peer review

- Open access: articles freely available online

- High visibility within the field

- Retaining the copyright to your article

Submit your next manuscript at $\boldsymbol{\nabla}$ springeropen.com 